\title{
2003 National Survey of Children's Health
}

\section{CHILD HEALTH \& HEALTH CARE MEASURES}

\section{SAS CODEBOOK}
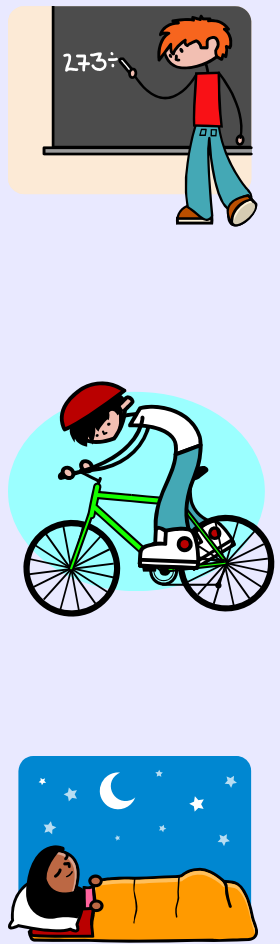

Version 1.1, June 2008
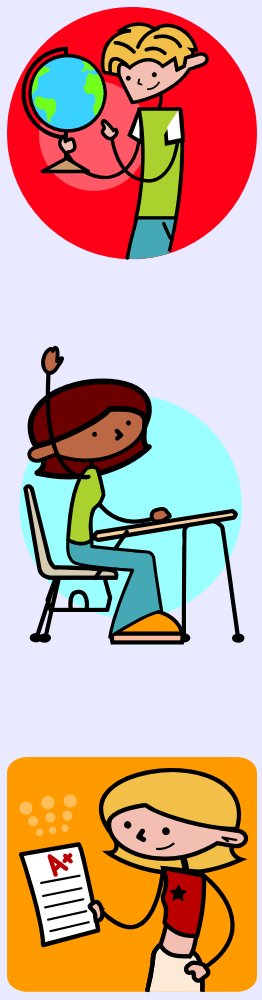



\section{Table of Contents}

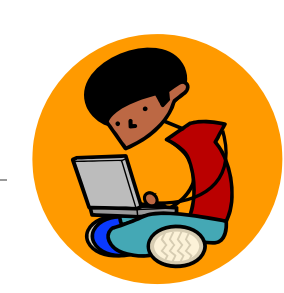

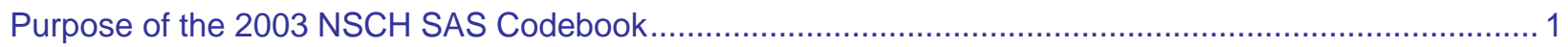

The Data Resource Center for Child and Adolescent Health ............................................................. 1

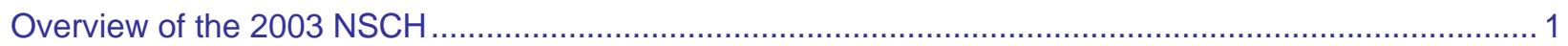

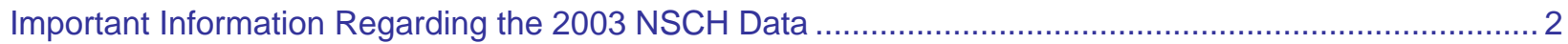

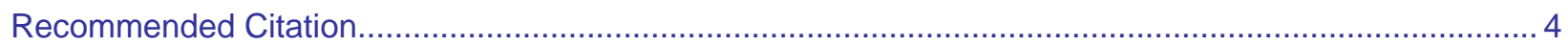

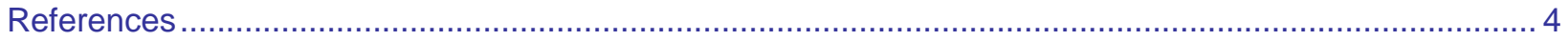

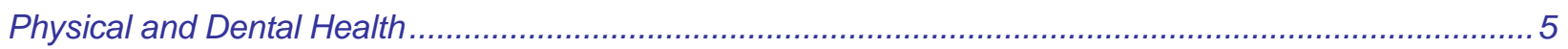

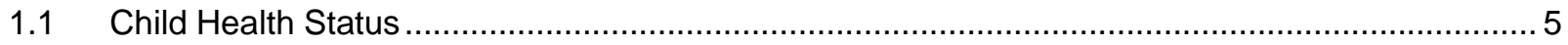

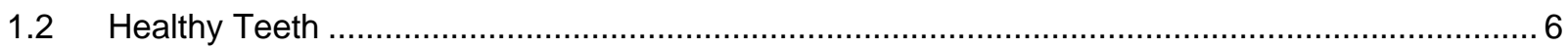

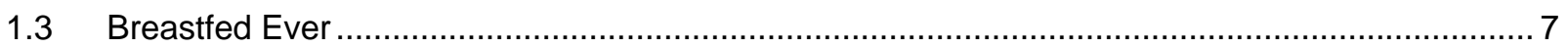

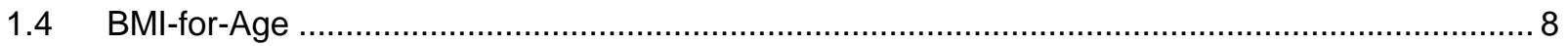

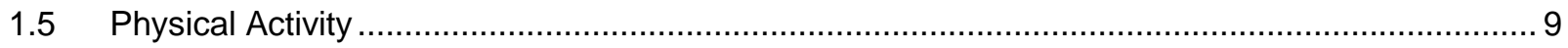

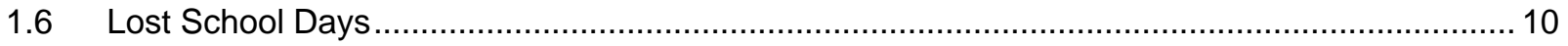

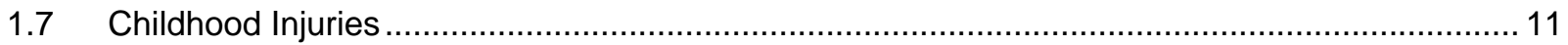

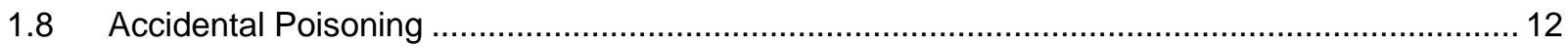

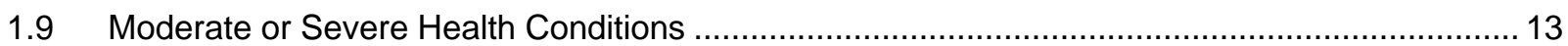

1.10 Impact of Asthma of Child: asthma-related health issues during the past 12 months .............. 14

1.10a Impact of Asthma on Child: no asthma-related health issues during the past 12 months .......... 15

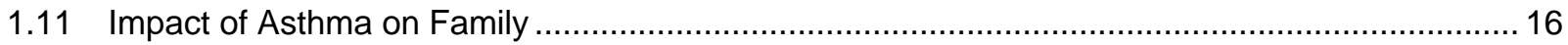

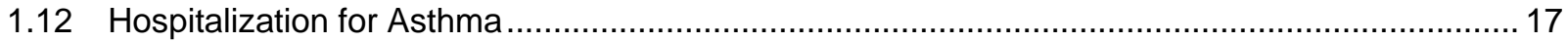

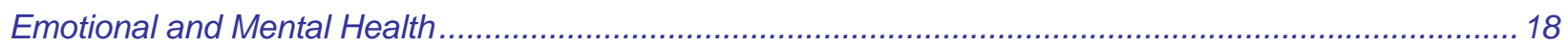

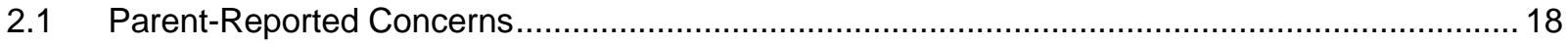




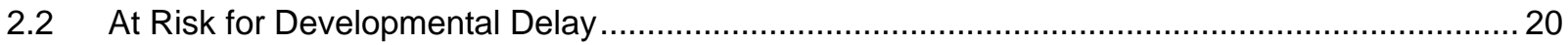

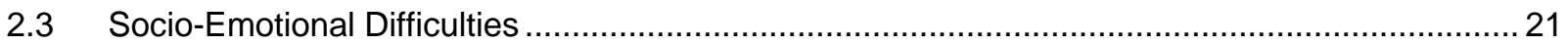

2.4 Impact of Children's Socio-Emotional Difficulties on Family ................................................ 22

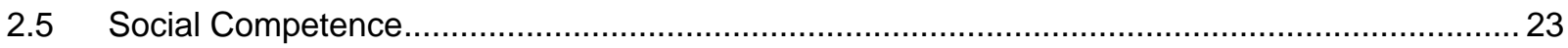

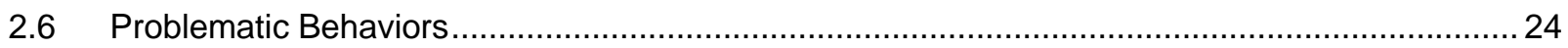

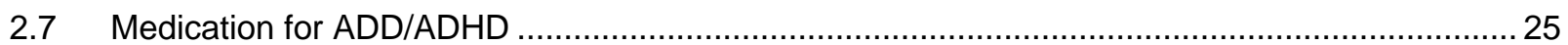

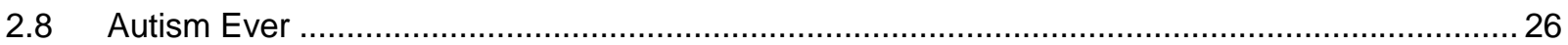

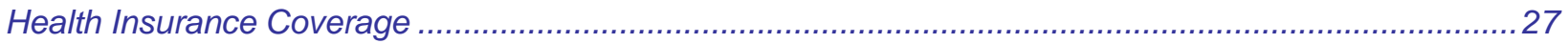

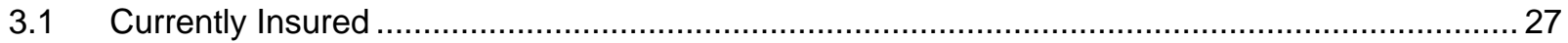

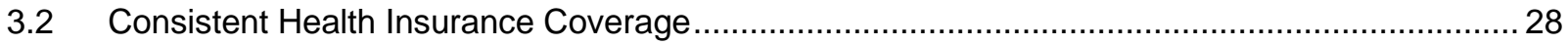

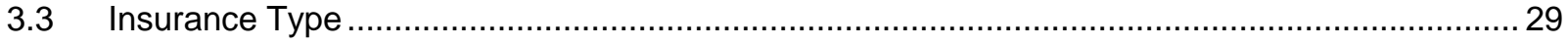

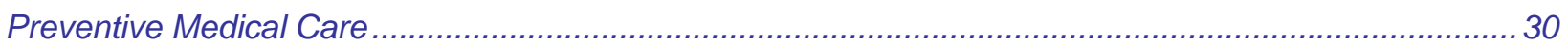

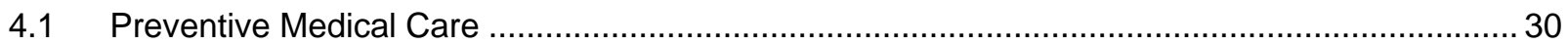

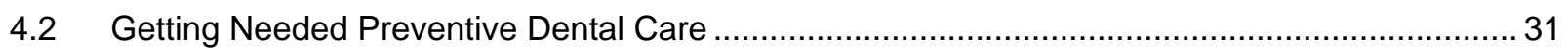

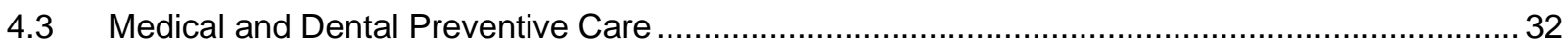

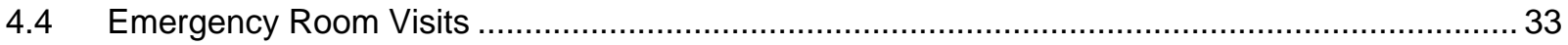

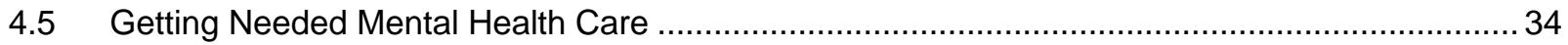

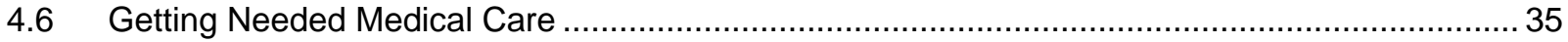

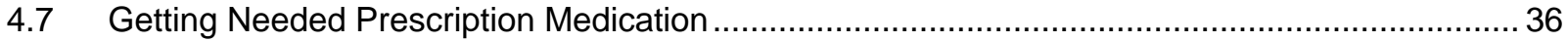

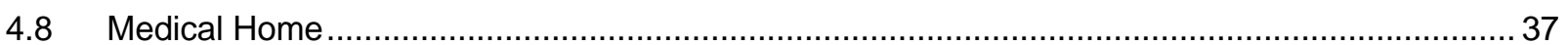

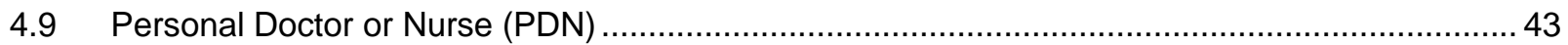

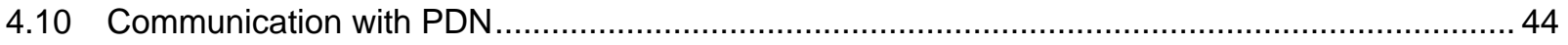

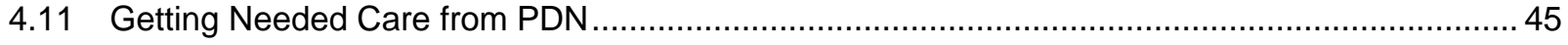

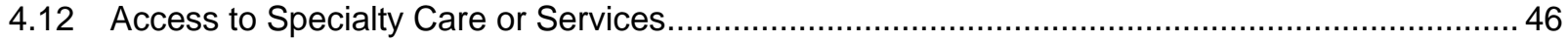

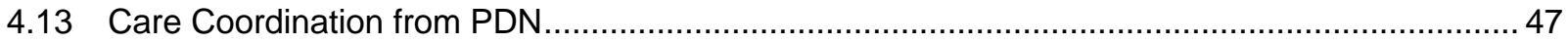

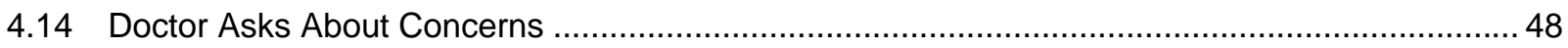




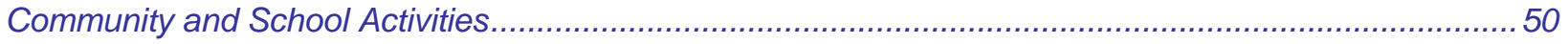

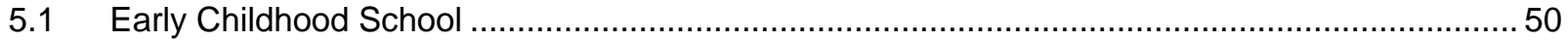

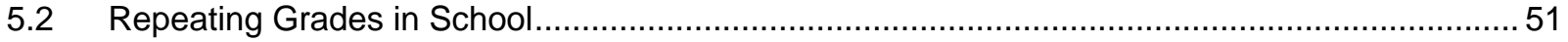

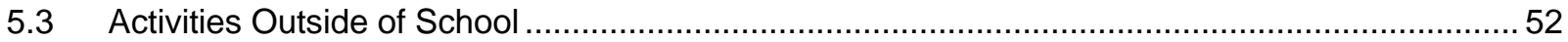

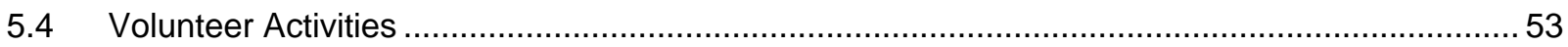

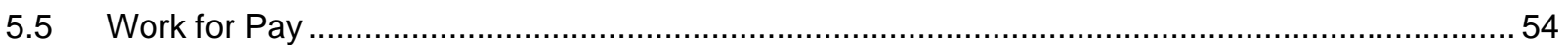

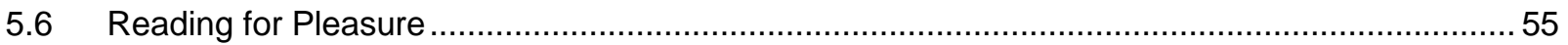

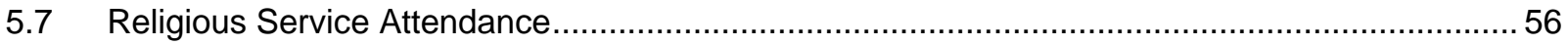

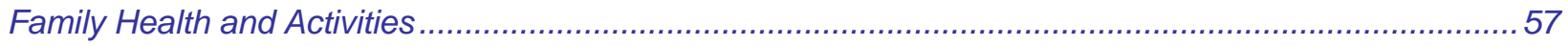

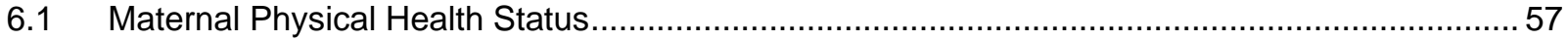

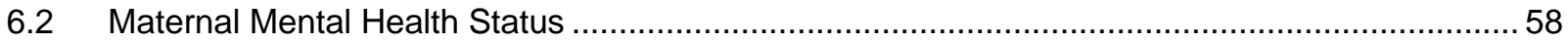

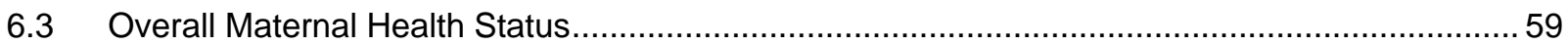

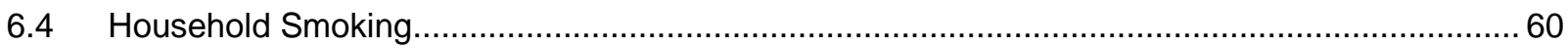

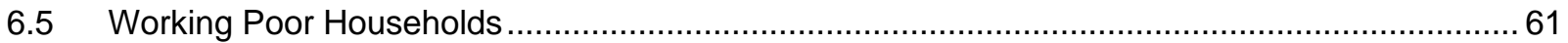

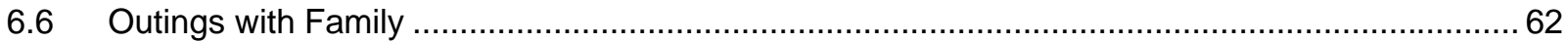

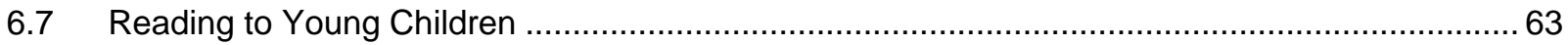

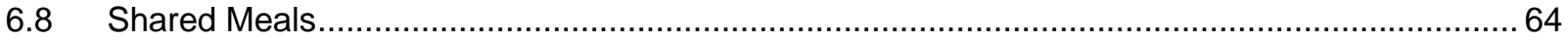

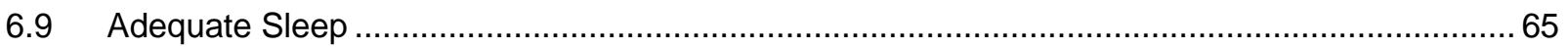

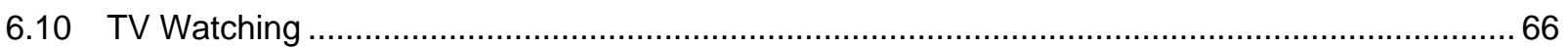

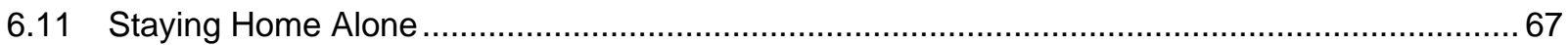

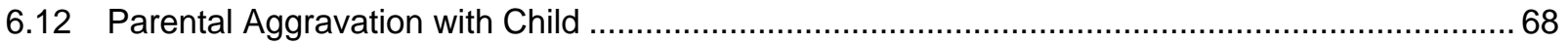

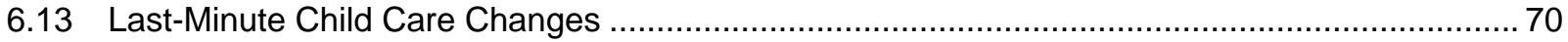

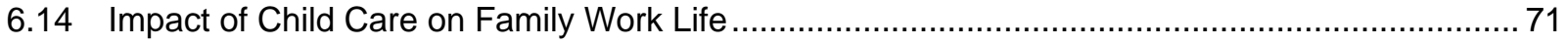

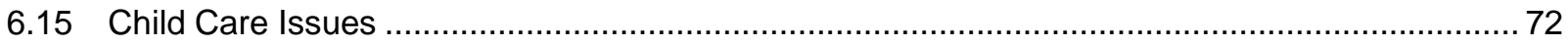

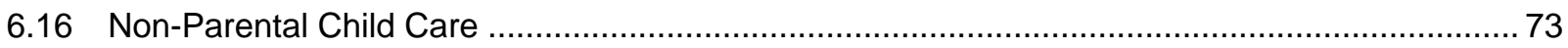




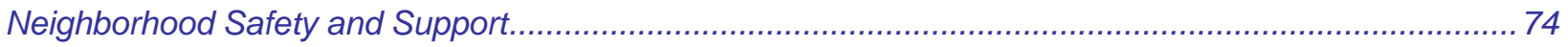

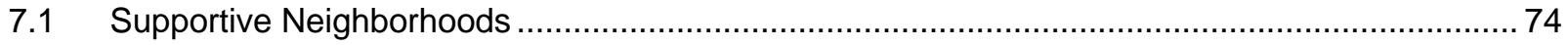

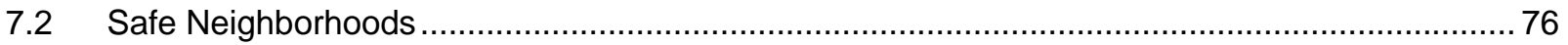

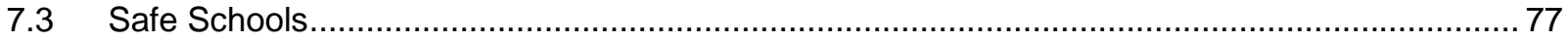

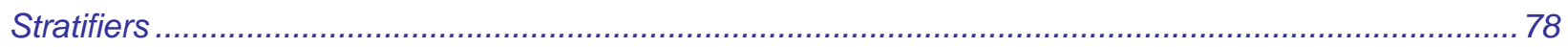

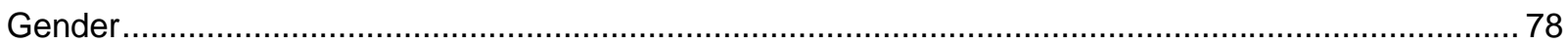

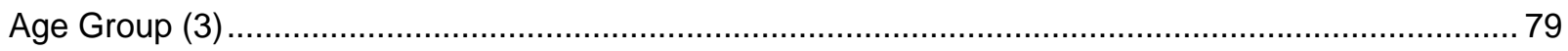

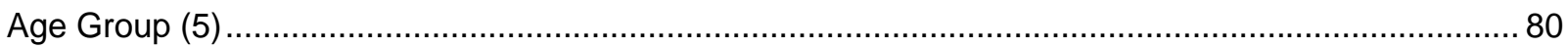

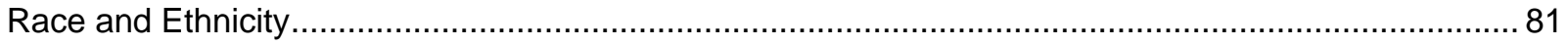

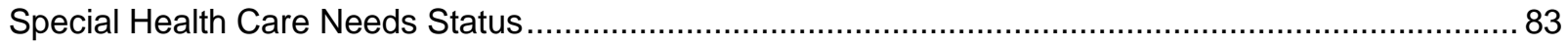

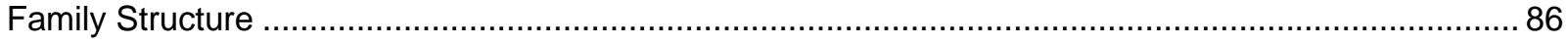

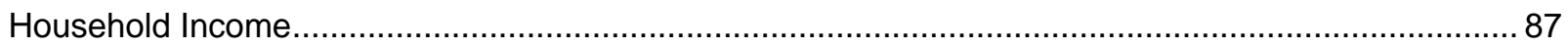

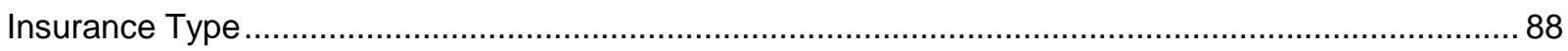

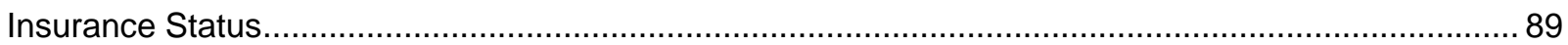

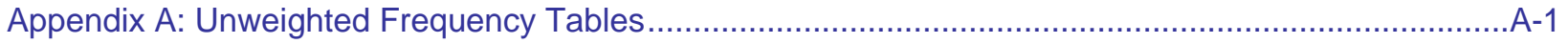

Appendix B: Medical Home Component and Interim Variables............................................................ 


\section{Acknowledgements}

This publication represents the work of the Child and Adolescent Health Measurement Initiative (CAHMI), in collaboration with Health Systems Research, Inc., an Altarum Company (HSR). The CAHMI would like to extend a special thank you to the Federal Maternal and Child Health Bureau for their support of the Data Resource Center for Child and Adolescent Health (DRC) and for the availability of the national and State-level survey data upon which the indicator code set forth in this document is based.

We also recognize the National Center for Health Statistics (NCHS) SLAITS team who led the data collection and development of the initial code for many DRC indicators. The indicators outlined here represent the intellectual leadership of many DRC advisors and experts nationally and in States. In addition, those researchers whom the CAHMI worked with to assist in replicating the code used to develop the DRC indicators in their own research have been very helpful to us in formulating indicator methods and the development of this codebook.

Special thanks to Kathy Newton and Debra Read of the CAHMI and Stacy Gleason and Holly Doggett of HSR for their effort in producing of this codebook, and to Stephen Blumberg, PhD, and his colleagues at the NCHS for their continued collaboration in making sure the code is right! 



\section{Purpose of the 2003 NSCH SAS Codebook}

This codebook contains SAS program statements that can be applied to the public use data file for the 2003 National Survey of Children's Health (NSCH) to create the Key Child Health

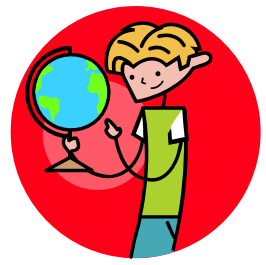
Indicators and variables used to stratify these items in the interactive data query feature of the Data Resource Center for Child and Adolescent Health (DRC), located online at www.childhealthdata.org.

The codebook is designed as a resource for researchers and analysts who are interested in understanding and replicating the Key Child Health Indicator variables developed from the $2003 \mathrm{NSCH}$ for the Data Resource Center for Child and Adolescent Health (DRC). Its purpose is to expedite research by helping to standardize and improve the comparability of information derived from the $2003 \mathrm{NSCH}$.

The DRC maintains a complete $2003 \mathrm{NSCH}$ indicator data set, available in either SAS or SPSS format. The data set includes all 65 Key Child Health Indicators, indicators pertinent to Healthy People 2010 goals, and demographic and individual question variables. To request a copy, contact the Child and Adolescent Health Measurement Initiative (CAHMI) at cahmi@ohsu.edu.

\section{The Data Resource Center for Child and Adolescent Health}

The Data Resource Center for Child and Adolescent Health (DRC) is an easy-to-use public Web site that eliminates barriers and reduces time and resources needed to obtain key findings on the health and health care of children, youth and families in the United States. The DRC is sponsored by the Maternal and Child Health Bureau and is led by The Child and Adolescent Health Measurement Initiative (CAHMI) based at the Oregon Health and Science University in Portland, Oregon. It is located online at www.childhealthdata.org.

The main feature of the DRC is an interactive data search tool that includes over 100 standardized indicators from the two extensive national and State-based surveys on the health and health care of children, youth and families: the 2001 National Survey of Children with Special Health Care Needs (NSCSHCN), and the 2003 National Survey of Children's Health (NSCH). Data search results can be stratified by the age, race/ethnicity, income and other pertinent characteristics of children and youth nationally or for individual States. Links to the survey instruments, sampling diagrams, methods reports and other survey specific resources are included on the DRC Web site.

\section{Overview of the $2003 \mathrm{NSCH}$}

The 2003 National Survey of Children's Health is a national telephone survey conducted during 20032004 by the Centers for Disease Control and Prevention, National Center for Health Statistics, and sponsored by the U.S. Department of Health and Human Services Administration, Maternal and Child Health Bureau. The survey is designed to (1) estimate national and State-level prevalence for a variety of child health indicators, (2) generate information about children, families, and neighborhoods to help guide policymakers, advocates, and researchers, (3) provide baseline estimates for Federal and State 
performance measures, Healthy People 2010 objectives and States' needs assessments, and (4) complement the National Survey of Children with Special Health Care Needs (NS-CSHCN).

The NSCH provides a broad range of information about children's health and well-being collected in a manner that allows comparisons among States as well as nationally. A total of 102,353 surveys completed nationally by parents/caregivers of children and youth ages 0-17 years - between 1,483 and 2,241 surveys per State - were collected. Survey results are weighted to represent the population of non-institutionalized children and youth ages 0-17 years in each State. Topics include demographics, physical and mental health status, health insurance, access and use of health care services, medical home, early childhood-specific information ( $0-5$ years), middle childhood- and adolescent-specific information (6-17 years), family health and activities, parental health status and perceptions of neighborhood characteristics.

The public use data file for the $2003 \mathrm{NSCH}$, along with relevant background information and documentation, can be obtained online at www.cdc.gov/nchs/about/major/slaits/nsch.htm. Additional information and results from the NSCH are available in the DRC at www.childhealthdata.org.

\section{Important Information Regarding the 2003 NSCH Data}

\section{Weighting}

Each record in the $2003 \mathrm{NSCH}$ public release data set is assigned a sampling weight, which is composed of a base sampling weight and a poststratification adjustment to match the child population in each State. The base sampling weight adjusts for number of residential telephone lines and sources of nonresponse. The interview response weights are further adjusted to match the age, race/ethnicity and gender distributions in the child population for each State based on July 2003 Census Bureau estimates. The poststratification procedure also adjusts for the number of households without telephones in each State. The variable WEIGHT_I, included in the public use data file, weights the survey responses to reflect the non-institutionalized population of children and youth ages 0-17 in each State.

\section{Variance Estimation}

Use of statistical software with the capacity to take into account the complex sampling design of the survey is necessary to appropriately calculate the variances and associated standard errors and confidence intervals required for accurate statistical hypothesis testing. Computer programs capable of variance estimation for complex sample designs include SUDAAN, SAS V 9.0+, STATA, WesVar, and SPSS Complex Samples.

The NSCH is designed to provide independent data sets for each of the 50 States and the District of Columbia. Subsetting the survey data to a particular State does not compromise the design structure of the survey. However, subsetting further to a specific population subgroup (age group, ethnicity or race, or poverty level, for example) within or across States will lead to incorrect standard error estimation. Most software packages that analyze complex sample data will incorrectly compute variances for subsetted data when the sample design is compromised because complete design information is not available. To 
avoid this problem, use a subpopulation procedure such as SUDAAN's SUBPOPN option that allows for the targeting of specific subpopulations for analysis while retaining the full sample design information. The only substate geographic information included in the NSCH public use data set is a variable for Metropolitan Statistical Area (MSA) status. The MSA status information is available for the 35 States in which the population is at least 500,000 in both categories (MSA and non-MSA). Zip code data is collected with the $\mathrm{NSCH}$; however, this information is not released in the public use data set due to confidentiality restrictions. The confidential zip code data can be made available to researchers for on site analysis at the Data Research Center of the National Center for Health Statistics (NCHS) in Hyattsville, MD. The process requires submitting a proposal to the NCHS. For more information, see www.cdc.gov/nchs/r\&d/rdc.htm.

\section{Interpretation of Results}

The respondents to the NSCH survey are parents; however, the results are weighted to reflect the population of children and youth ages 0-17, not parents or families. Thus, results are reported in terms of children/youth, even if the question refers to the parents or family. For example, when reporting on maternal health status it is correct to say: "... percentage of children whose mothers are in excellent or very good health," NOT “...percentage of mothers who are in excellent or very good health...."

The condition-specific information in this survey is based on parent report, not clinical diagnosis. Height and weight information used to calculate the Body Mass Index (BMI)-for-age indicator is also derived from parent report, not biometric measurement.

Pay careful attention to the valid denominator of responders to specific questions when interpreting results for this survey. Although most of the questions were asked for all children ages 0-17, two sections of the survey targeted specific age groups: early childhood (ages 0-5) and middle childhood and adolescence (ages 6-17). In several parts of the survey, follow-up questions are asked only if parents gave a specific response to an earlier question.

\section{Treatment of Unknown/Missing Values}

Responses of "Don't know" or "Refused" and system missing data are not included in the denominators used in the construction of the variables in this codebook. In nearly all cases, the unknown values make up less than 1 percent of the responses to any single question. However, a few questions have higher proportions of unknown values, most notably Household Income (about 10 percent unknown values nationally), and BMI-for-age (6.5 percent nationally).

\section{Verification}

Unweighted frequency distributions for all of the variables in this codebook are located in Appendix A as a resource for cross checking results. Results may also be compared with results from the interactive data query in the DRC. 


\section{Additional Information on Survey Design and Methodology}

The NCHS report, Design and Operation of the National Survey of Children's Health, 2003 (2) is an excellent resource. This document includes detailed descriptions of questionnaire development, sample selection and weighting procedures, events that affected data collection, adjustments made to protect data confidentiality, and more. View or download the methods report from the DRC Web site for the $\mathrm{NSCH}$ at www.nschdata.org/content/LearnAboutTheSurvey.aspx or the NSCH Web Site at www.cdc.gov/nchs/data/slaits/NSCH_Methodology_Report.pdf.

\section{Recommended Citation}

Publication or presentation of any analysis involving variables constructed using code from this document in any form should be appropriately cited. The following format is recommended:

Child and Adolescent Health Measurement Initiative (CAHMI), 2003 National Survey of Children's Health SAS Codebook, Data Resource Center for Child and Adolescent Health, www.childhealthdata.org.

\section{References}

1. Child and Adolescent Health Measurement Initiative (CAHMI), "The Children with Special Health Care Needs (CSHCN) Screener." 2002, www.ohsudoernbecher.com/CAHMI/CSHCN-screener.pdf .

2. Blumberg SJ, Olson L, Frankel MR, Osborn L, Srinath KP, Giambo P. "Design and operation of the National Survey of Children's Health, 2003." National Center for Health Statistics. Vital Health Stat 1(43). 2005.

3. U.S. Department of Health and Human Services, Health Resources and Services Administration, Maternal and Child Health Bureau. "The National Survey of Children's Health 2003." Rockville, Maryland: U.S. Department of Health and Human Services, 2005.

4. Department of Health and Human Services, Centers for Disease Control and Prevention, National Center for Health Statistics, Division of Health Interview Statistics, Special Population Surveys Branch. "Progress toward implementing community-based systems of services for children with special health care needs: Summary tables form the National Survey of Children with Special Health Care Needs, 2001." April 2003. 


\begin{tabular}{|c|c|}
\hline INDICATOR 1.1 & In general, how would you describe (child's name)'s health? \\
\hline Survey Items Used & S2Q01 \\
\hline Numerators & $\begin{array}{l}\text { - } \quad \text { Children whose health status is excellent or very good } \\
\text { - } \quad \text { Children whose health status is good } \\
\text { - } \quad \text { Children whose health status is fair or poor }\end{array}$ \\
\hline Denominator & Children ages $0-17$ years \\
\hline Description & $\begin{array}{l}\text { Percent of children ages } 0-17 \text { whose health status is excellent/very good, } \\
\text { good, or fair/poor, as reported by their parents. }\end{array}$ \\
\hline \multicolumn{2}{|l|}{ Notes for Data-Users } \\
\hline \multicolumn{2}{|c|}{ SAS Code and Annotation } \\
\hline \multicolumn{2}{|l|}{ Format } \\
\hline \multicolumn{2}{|c|}{ 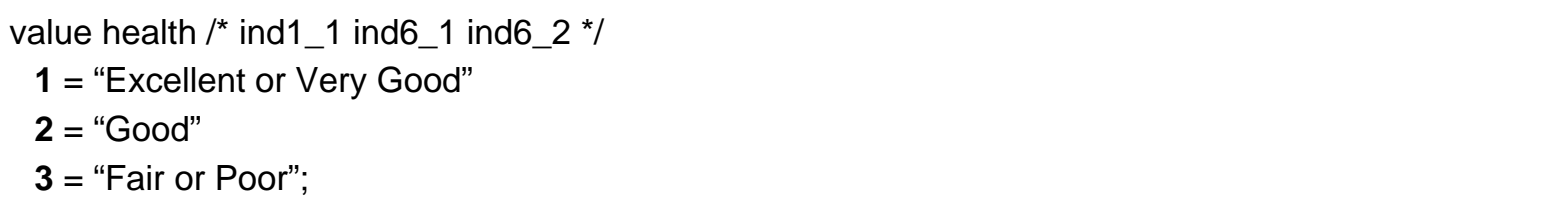 } \\
\hline \multicolumn{2}{|l|}{ Code } \\
\hline \multicolumn{2}{|c|}{ 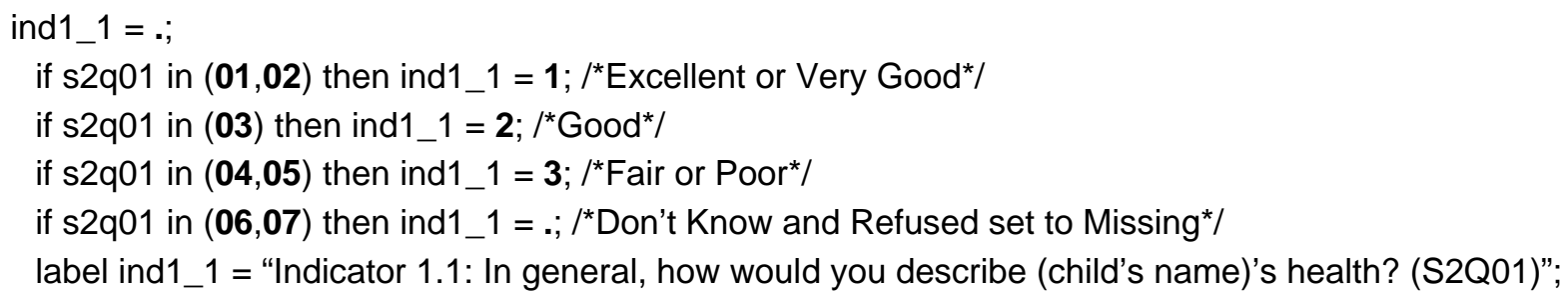 } \\
\hline
\end{tabular}




\begin{tabular}{|c|c|}
\hline INDICATOR 1.2 & How would you describe the condition of (child's name)'s teeth? \\
\hline Survey Items Used & S2Q54 \\
\hline Numerators & $\begin{array}{l}\text { - Children/youth whose teeth are in excellent or very good condition } \\
\text { - } \quad \text { Children/youth whose health status are in good condition } \\
\text { - } \quad \text { Children/youth whose health status are in fair or poor condition }\end{array}$ \\
\hline Denominator & Children/youth ages 1-17 years who have one or more natural teeth \\
\hline Description & $\begin{array}{l}\text { Percent of children/youth ages 1-17 whose teeth are in excellent/very good } \\
\text { condition, good condition, or fair/poor condition, as reported by their parents. }\end{array}$ \\
\hline \multicolumn{2}{|l|}{ Notes for Data-Users } \\
\hline \multicolumn{2}{|c|}{$\begin{array}{l}\text { Children under } 1 \text { and children/youth } v \\
\text { has no natural teeth) were not include }\end{array}$} \\
\hline \multicolumn{2}{|c|}{ SAS Code and Annotation } \\
\hline \multicolumn{2}{|l|}{ Format } \\
\hline \multicolumn{2}{|c|}{$\begin{array}{l}\text { value teeth_di /*ind1_2 }{ }^{*} \\
\mathbf{1}=\text { "Excellent or very good condition" } \\
\mathbf{2}=\text { "Good condition" } \\
\mathbf{3}=\text { "Fair or poor condition"; }\end{array}$} \\
\hline \multicolumn{2}{|l|}{ Code } \\
\hline \multicolumn{2}{|c|}{ 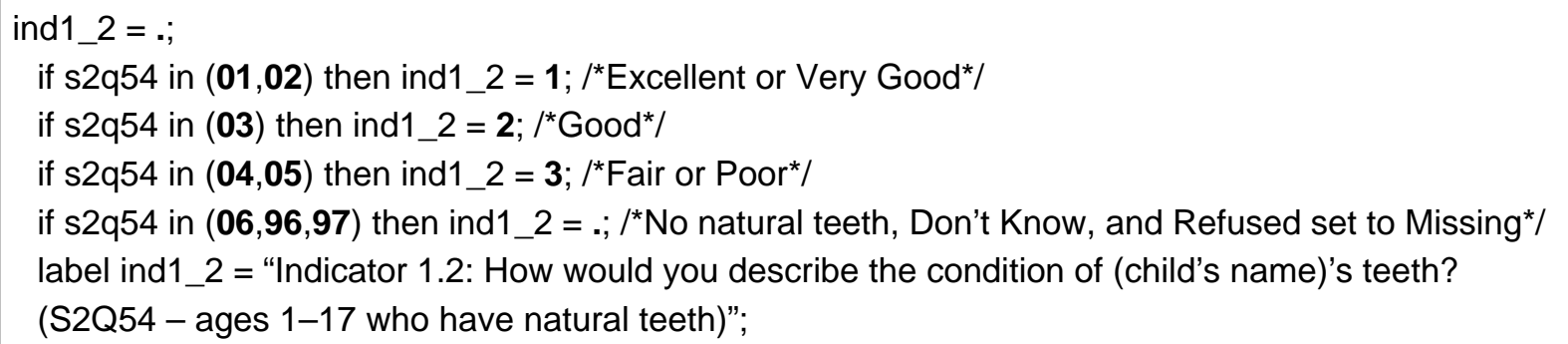 } \\
\hline
\end{tabular}




\begin{tabular}{|c|c|}
\hline INDICATOR 1.3 & Was (child's name) ever breastfed or fed breast milk? \\
\hline Survey Items Used & S6Q59 \\
\hline Numerator & Young children who were breastfed or fed breast milk for any length of time \\
\hline Denominator & Children ages $0-5$ years \\
\hline Description & $\begin{array}{l}\text { Percent of children ages } 0-5 \text { who were ever breastfed or fed breast milk } \\
\text { (S6Q59 = Yes). }\end{array}$ \\
\hline \multicolumn{2}{|l|}{ Notes for Data-Users } \\
\hline \multicolumn{2}{|c|}{ SAS Code and Annotation } \\
\hline \multicolumn{2}{|l|}{ Format } \\
\hline \multicolumn{2}{|c|}{$\begin{array}{l}\text { value yn /*ind1_3 ind1_7 ind1_8 ind2_8 ind3_1 ind4_6 ind4_9 ind5_2 ind6_14*/ } \\
\mathbf{1}=\text { = "Yes" } \\
\mathbf{2}=\text { "No"; }\end{array}$} \\
\hline \multicolumn{2}{|l|}{ Code } \\
\hline \multicolumn{2}{|c|}{ 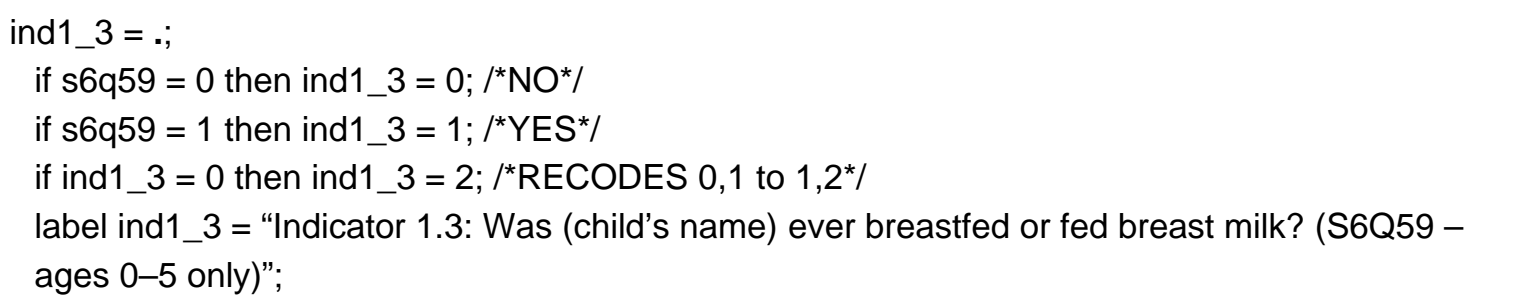 } \\
\hline
\end{tabular}




\begin{tabular}{|c|c|}
\hline INDICATOR 1.4 & $\begin{array}{l}\text { What is the weight status of children/youth based on Body Mass Index- } \\
\text { for-Age (BMl-for-age)? }\end{array}$ \\
\hline Survey Items Used & S2Q02, S2Q03, AGEYR_CHILD \\
\hline Numerators & $\begin{array}{l}\text { - } \quad \text { Children/youth who are overweight } \\
\text { - } \quad \text { Children/youth who are at risk of being overweight } \\
\text { - } \quad \text { Children/youth who are normal weight } \\
\text { - } \quad \text { Children/youth who are underweight }\end{array}$ \\
\hline Denominator & Children/youth ages $10-17$ years \\
\hline Description & $\begin{array}{l}\text { Percent of children/youth ages } 10-17 \text { who are overweight, at risk of overweight, } \\
\text { normal weight, or underweight according to BMI-for-age calculated from parent } \\
\text { report of child's height and weight. }\end{array}$ \\
\hline \multicolumn{2}{|l|}{ Notes for Data-Users } \\
\hline \multicolumn{2}{|c|}{$\begin{array}{l}\text { BMI-for-age calculated from the NSCH } 2003 \text { is based on parent-reported height and weight of children. } \\
\text { Calculation of BMI-for-age is usually based on the age of the child in months. Because age was only } \\
\text { reported in years for this survey, children were assumed to be at the midpoint of the age-year for } \\
\text { purposes of calculating BMI-for-age. It should be recognized that height and weight were based on the } \\
\text { parents' reports and were not independently measured. Height and weight estimates based on parent } \\
\text { reporting from the NSCH } 2003 \text { were compared to national estimates based on independent } \\
\text { measurement from the National Health and Nutrition Examination Survey. This comparison revealed } \\
\text { that, for children under } 10 \text { years of age, height was generally underreported and weight was generally } \\
\text { overreported. Therefore, BMI for children under } 10 \text { years of age has not been included as part of this } \\
\text { indicator. For more information about BMI-for-age, go to: www.cdc.gov/nccdphp/dnpa/bmi/bmi-for- } \\
\text { age.htm }\end{array}$} \\
\hline \multicolumn{2}{|c|}{$\begin{array}{l}\text { The number of unknown values for this variable is unusually high }(6.5 \%) \text {. Unknown values are not } \\
\text { included in the denominator; weighted population counts may be underestimated. }\end{array}$} \\
\hline \multicolumn{2}{|c|}{ SAS Code and Annotation } \\
\hline \multicolumn{2}{|l|}{ Format } \\
\hline \multicolumn{2}{|c|}{$\begin{array}{l}\text { value bmi /*ind1_4*/ } \\
\mathbf{1}=\text { = "Underweight" } \\
\mathbf{2}=\text { "Normal Weight" } \\
\mathbf{3}=\text { ="At Risk for Overweight" } \\
\mathbf{4}=\text { = OVverweight"; }\end{array}$} \\
\hline \multicolumn{2}{|l|}{ Code } \\
\hline \multicolumn{2}{|c|}{$\begin{array}{l}\text { ind1_4 = bmiclass; } \\
\text { if ageyr_child <10 then ind1_4 =; } \\
\text { label ind1_4 = "Indicator 1.4: What is the weight status of children/youth ages } 10-17 \text { based on BMI- } \\
\text { for-age? (derived)"; }\end{array}$} \\
\hline
\end{tabular}




\begin{tabular}{|c|c|}
\hline INDICATOR 1.5 & $\begin{array}{l}\text { During the past week, on how many days did (child's name) exercise or } \\
\text { participate in physical activity for at least } 20 \text { minutes that made him/her } \\
\text { sweat and breathe hard? }\end{array}$ \\
\hline Survey Items Used & S7Q21 \\
\hline Numerators & $\begin{array}{l}\text { - Children/youth who exercised on no days during the past week } \\
\text { - Children/youth who exercised on 1-3 days during the past week } \\
\text { - Children/youth who exercised on 4-6 days during the past week } \\
\text { - Children/youth who exercised every day during the past week }\end{array}$ \\
\hline Denominator & Children/youth ages 6-17 years \\
\hline Description & $\begin{array}{l}\text { Percent of children/youth ages } 6-17 \text { who did not engage in vigorous physical } \\
\text { activity or who engaged in vigorous physical activity for } 20 \text { minutes or more on } \\
1-3 \text { days, } 4-6 \text { days, or every day of the past week }\end{array}$ \\
\hline \multicolumn{2}{|l|}{ Notes for Data-Users } \\
\hline \multicolumn{2}{|c|}{ SAS Code and Annotation } \\
\hline \multicolumn{2}{|l|}{ Format } \\
\hline $\begin{array}{l}\text { value exercise /*ind1 } \\
\mathbf{1}=\text { = } 0 \text { days" } \\
\mathbf{2}=\text { = } 1-3 \text { days" } \\
\mathbf{3}=\text { "4-6 days" } \\
\mathbf{4}=\text { "Every day"; }\end{array}$ & \\
\hline \multicolumn{2}{|l|}{ Code } \\
\hline $\begin{array}{l}\text { ind1_5 }=\text {; } \\
\text { if s7q21 eq } 0 \text { then i } \\
\text { if } 1<=\text { s7q21<= } \\
\text { if } 4<=s 7 q 21<=6 \\
\text { if } s 7 q 21 \text { eq } 7 \text { then i } \\
\text { ind1_5 } 5 \text { ind1_5 } 5+1 \\
\text { if s7q21 in }(96,97) t \\
\text { label ind1_5 = "Indi } \\
\text { participate in physic } \\
\text { (S7Q21 - ages } 6-1\end{array}$ & $\begin{array}{l}1 \_5=0 \\
\text { en ind1_5 = 1; } \\
\text { en ind1_5 = } 2 \\
1 \_5=3 \\
\text { n ind1_5 = ; } \\
\text { or 1.5: During the past week, on how many days did (child's name) exercise or } \\
\text { activity for at least } 20 \text { minutes that made him/her sweat and breathe hard? } \\
\text { only)"; }\end{array}$ \\
\hline
\end{tabular}




\begin{tabular}{|c|c|}
\hline INDICATOR 1.6 & $\begin{array}{l}\text { During the past } 12 \text { months, about how many days did (child's name) miss } \\
\text { school because of illness or injury? }\end{array}$ \\
\hline Survey Items Used & S7Q02 \\
\hline Numerators & $\begin{array}{l}\text { - } \quad \text { Children/youth who missed no school days due to illness/injury } \\
\text { - } \quad \text { Children/youth who missed 1-5 school days due to illness/injury } \\
\text { - } \quad \text { Children/youth who missed 6-10 school days due to illness/injury } \\
\text { - } \quad \text { Children/youth who missed } 11 \text { or more school days due to illness/injury }\end{array}$ \\
\hline Denominator & Children/youth ages 6-17 years \\
\hline Description & $\begin{array}{l}\text { Percent of children/youth ages } 6-17 \text { who did not miss school, or who missed } \\
\text { school } 1-5 \text { days, } 6-10 \text { days, or } 11 \text { or more days because of illness or injury. }\end{array}$ \\
\hline \multicolumn{2}{|l|}{ Notes for Data-Users } \\
\hline \multicolumn{2}{|c|}{ SAS Code and Annotation } \\
\hline \multicolumn{2}{|l|}{ Format } \\
\hline $\begin{array}{l}\text { value miss_sch } \\
\begin{array}{l}\mathbf{1}=\text { "0 days" } \\
\mathbf{2}=\text { "1-5 days" } \\
\mathbf{3}=\text { "6-10 days" } \\
\mathbf{4}=\text { "11 + days"; }\end{array}\end{array}$ & \\
\hline \multicolumn{2}{|l|}{ Code } \\
\hline $\begin{array}{l}\text { ind1_6 = ; } \\
\text { if } s 7 q 02 r \text { in }(994,99 \\
\text { if } s 7 q 02 r=000 \text { ther } \\
\text { if } 001<=s 7 q 02 r< \\
\text { if } 006<=s 7 q 02 r< \\
\text { if } 011<=s 7 q 02 r< \\
\text { label ind1_6 = "Indi } \\
\text { miss school becaus }\end{array}$ & 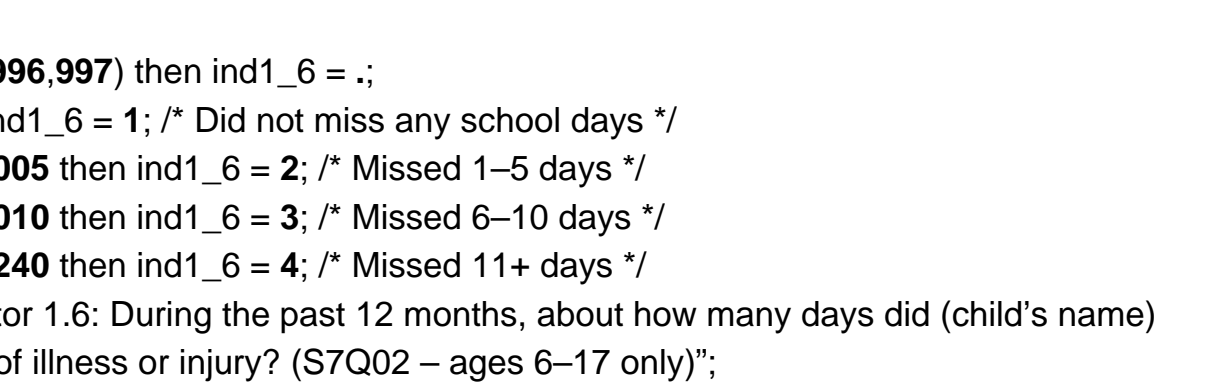 \\
\hline
\end{tabular}




\begin{tabular}{|c|c|}
\hline INDICATOR 1.7 & $\begin{array}{l}\text { During the past } 12 \text { months, has (child's name) been injured and required } \\
\text { medical attention? }\end{array}$ \\
\hline Survey Items Used & S6Q55 \\
\hline Numerator & $\begin{array}{l}\text { Young children who experienced injuries requiring medical attention during the } \\
\text { past } 12 \text { months }\end{array}$ \\
\hline Denominator & Children ages $0-5$ years \\
\hline Description & $\begin{array}{l}\text { Percent of children ages } 0-5 \text { who experienced injuries requiring medical } \\
\text { attention during the past } 12 \text { months (S6Q55 = Yes). }\end{array}$ \\
\hline \multicolumn{2}{|l|}{ Notes for Data-Users } \\
\hline \multicolumn{2}{|c|}{ SAS Code and Annotation } \\
\hline \multicolumn{2}{|l|}{ Format } \\
\hline \multicolumn{2}{|c|}{$\begin{array}{l}\text { value yn /*ind1_3 ind1_7 ind1_8 ind2_8 ind3_1 ind4_6 ind4_9 ind5_2 ind6_14*/ } \\
\mathbf{1}=\text { ="Yes" } \\
\mathbf{2}=\text { "No"; }\end{array}$} \\
\hline \multicolumn{2}{|l|}{ Code } \\
\hline \multicolumn{2}{|c|}{$\begin{array}{l}\text { ind1_7 = s6q55; } \\
\text { if s6q55 in }(\mathbf{6}, 7) \text { then ind1_7 = ; } \\
\text { if ind1_7 = } 0 \text { then ind1_7 = 2; /* recodes from } 0,1 \text { to } 1,2 * / \\
\text { label ind1_7 = "Indicator } 1.7 \text { : During the past } 12 \text { months, has (child's name) been injured and required } \\
\text { medical attention? (S6Q55 - ages } 0-5 \text { only)"; }\end{array}$} \\
\hline
\end{tabular}




\begin{tabular}{|c|c|}
\hline INDICATOR 1.8 & $\begin{array}{l}\text { During the past } 12 \text { months, has (child's name) been poisoned by accident } \\
\text { and required medical attention? }\end{array}$ \\
\hline Survey Items Used & S6Q57 \\
\hline Numerator & $\begin{array}{l}\text { Young children who required medical attention for accidental poisoning during } \\
\text { past } 12 \text { months }\end{array}$ \\
\hline Denominator & Children ages $0-5$ years \\
\hline Description & $\begin{array}{l}\text { Percent of children ages 0-5 who were accidentally poisoned and required } \\
\text { medical attention during past } 12 \text { months (S6Q57 = Yes). }\end{array}$ \\
\hline \multicolumn{2}{|l|}{ Notes for Data-Users } \\
\hline \multicolumn{2}{|c|}{ SAS Code and Annotation } \\
\hline \multicolumn{2}{|l|}{ Format } \\
\hline \multicolumn{2}{|c|}{$\begin{array}{l}\text { value yn /*ind1_3 ind1_7 ind1_8 ind2_8 ind3_1 ind4_6 ind4_9 ind5_2 ind6_14*/ } \\
\mathbf{1}=\text { "Yes" } \\
\mathbf{2}=\text { "No"; }\end{array}$} \\
\hline \multicolumn{2}{|l|}{ Code } \\
\hline \multicolumn{2}{|c|}{$\begin{array}{l}\text { ind1_8 = s6q57; } \\
\text { if s6q57 in }(\mathbf{6 , 7}) \text { then ind1_8 }=. ; \\
\text { if ind1_8 = } \mathbf{0} \text { then ind1_8= } 2 ; \text { /*recodes from } 0,1 \text { to } 1,2^{*} / \\
\text { label ind1_8 = "Indicator } 1.8 \text { : During the past } 12 \text { months, has (child's name) been poisoned by } \\
\text { accident and required medical attention? (S6Q57 - ages } 0-5 \text {; national-level data only)"; }\end{array}$} \\
\hline
\end{tabular}




\begin{tabular}{|c|c|}
\hline INDICATOR 1.9 & $\begin{array}{l}\text { How many children/youth currently have health conditions described as } \\
\text { moderate or severe by their parents? }\end{array}$ \\
\hline Survey Items Used & S2Q04 - S2Q17; S2Q20 - S2Q42; S2Q47, S2Q49 - S2Q50 \\
\hline Numerator & $\begin{array}{l}\text { Children/youth with current health conditions rated by their parents as being } \\
\text { moderate or severe }\end{array}$ \\
\hline Denominator & Children/youth ages $0-17$ years \\
\hline Description & $\begin{array}{l}\text { Percent of children/youth ages } 0-17 \text { whose parents report that the child has } \\
\text { specified ongoing health condition(s) (CYSHCN and "yes" on one or more of } \\
\text { S2Q20 - S2Q42 or S2Q49) that the parents rate as moderate or severe } \\
\text { (S2Q47). }\end{array}$ \\
\hline \multicolumn{2}{|l|}{ Notes for Data-Users } \\
\hline \multicolumn{2}{|c|}{$\begin{array}{l}\text { For a child to qualify as having a "current health condition," the child's parent must report having ever } \\
\text { been told by a doctor or health professional that the child has one or more conditions listed in questions } \\
\text { S2Q20 - S2Q42 or S2Q49, AND the child must have a positive screen on the CSHCN Screener which } \\
\text { indicates that he/she currently experiences on-going health consequences from a medical, emotional, } \\
\text { developmental, or behavioral condition lasting } 12 \text { months or longer. }\end{array}$} \\
\hline \multicolumn{2}{|c|}{$\begin{array}{l}\text { Only children whose parents report having ever been told that the child has one or more of the } \\
\text { conditions asked about in questions S2Q20 - S2Q42 or S2Q49 are asked to rate the severity of the } \\
\text { health conditions on these lists that the child may either have now or have had in the past. Parents of } \\
\text { children who currently have health conditions that are not listed in questions S2Q20 - S2Q42 or S2Q49 } \\
\text { were not given the opportunity to rate the severity of their children's health problems. }\end{array}$} \\
\hline \multicolumn{2}{|c|}{ SAS Code and Annotation } \\
\hline \multicolumn{2}{|l|}{ Format } \\
\hline \multicolumn{2}{|c|}{$\begin{array}{l}\text { value cond } / * \text { ind } 1 \_9 * / \\
\mathbf{1}=\text { "No conditions reported" } \\
\mathbf{2}=\text { "Reported health conditions are minor or do not currently affect child" } \\
\mathbf{3}=\text { "Current health conditions rated moderate or sever by parents"; }\end{array}$} \\
\hline \multicolumn{2}{|l|}{ Code } \\
\hline & PENDING as of $1 / 31 / 07$ \\
\hline
\end{tabular}




\begin{tabular}{|c|c|}
\hline INDICATOR 1.10 & $\begin{array}{l}\text { How many children/youth experienced one or more asthma-related health } \\
\text { issues during the past } 12 \text { months? }\end{array}$ \\
\hline Survey Items Used & S2Q49, S2Q50, S2Q52 - S2Q53 \\
\hline Numerator & $\begin{array}{l}\text { Children/youth who still have asthma and also experienced one or more } \\
\text { asthma-related health issues during the past } 12 \text { months }\end{array}$ \\
\hline Denominator & Children/youth ages 0-17 years \\
\hline Description & $\begin{array}{l}\text { Percent of children/youth ages } 0-17 \text { whose parents report that the child still has } \\
\text { asthma and has been affected by asthma-related issues during the past } \\
12 \text { months. }\end{array}$ \\
\hline \multicolumn{2}{|l|}{ Notes for Data-Users } \\
\hline \multicolumn{2}{|c|}{$\begin{array}{l}\text { "Children with asthma-related health issues" are those whose parents were told at some point in time by } \\
\text { a doctor that the child had asthma (S2Q19 = Yes). In order to qualify as being affected by asthma } \\
\text { during the past year, children needed to still have asthma at the time of the survey (S2Q49 = Yes) AND } \\
\text { experience one or more of the following asthma-related health issues during the past } 12 \text { months: use of } \\
\text { asthma medication (S2Q52, response indicates use of medication within the past year), significant } \\
\text { health difficulties due to asthma (S2Q50 = Moderate or Severe), asthma attacks (S2Q52A = Yes), or } \\
\text { hospitalization for asthma (S2Q53 = Yes). }\end{array}$} \\
\hline \multicolumn{2}{|c|}{ SAS Code and Annotation } \\
\hline \multicolumn{2}{|l|}{ Format } \\
\hline \multicolumn{2}{|c|}{$\begin{array}{l}\text { value asth_iss /*ind1_10*/ } \\
\begin{aligned} \mathbf{1} & =\text { "No asthma reported" } \\
\mathbf{2} & =\text { "Asthma reported, but did not affect health during past } 12 \text { months" } \\
\mathbf{3} & =\text { "Affected by asthma-related health issues during the past } 12 \text { months"; }\end{aligned}\end{array}$} \\
\hline \multicolumn{2}{|l|}{ Code } \\
\hline & PENDING as of $1 / 31 / 07$ \\
\hline
\end{tabular}




\begin{tabular}{|c|c|}
\hline INDICATOR 1.10a & $\begin{array}{l}\text { How many children/youth currently have asthma and did not experience } \\
\text { asthma-related health issues during the past } 12 \text { months? }\end{array}$ \\
\hline Survey Items Used & S2Q49, S2Q50, S2Q52 - S2Q53 \\
\hline Numerator & $\begin{array}{l}\text { Children/youth who still have asthma have not experienced any significant } \\
\text { asthma-related health issues during the past } 12 \text { months }\end{array}$ \\
\hline Denominator & Children/youth ages 0-17 years \\
\hline Description & $\begin{array}{l}\text { Percent of children/youth ages } 0-17 \text { whose parents report that the child still has } \\
\text { asthma but have not been affected by asthma-related issues during the past } \\
12 \text { months. }\end{array}$ \\
\hline \multicolumn{2}{|c|}{ Notes for Data-Users } \\
\hline \multicolumn{2}{|c|}{$\begin{array}{l}\text { "Children with asthma-related health issues" are those whose parents were told at some point in time by } \\
\text { a doctor that the child had asthma (S2Q19 = Yes). In order to qualify as being affected by asthma } \\
\text { during the past year, children needed to still have asthma at the time of the survey (S2Q49 = Yes) AND } \\
\text { experience one or more of the following asthma-related health issues during the past } 12 \text { months: use of } \\
\text { asthma medication (S2Q52, response indicates use of medication within the past year), significant } \\
\text { health difficulties due to asthma (S2Q50 = Moderate or Severe), asthma attacks (S2Q52A = Yes), or } \\
\text { hospitalization for asthma (S2Q53 = Yes). }\end{array}$} \\
\hline \multicolumn{2}{|c|}{ SAS Code and Annotation } \\
\hline \multicolumn{2}{|l|}{ Format } \\
\hline \multicolumn{2}{|c|}{$\begin{array}{l}\text { value asth_cur /*ind1_10a*/ } \\
\mathbf{1}=\text { "No asthma reported" } \\
\mathbf{2}=\text { "Has asthma in the past, but do not have it any longer" } \\
\mathbf{3}=\text { "Current asthma did not affect health during the past } 12 \text { months" } \\
\mathbf{4}=\text { "Current asthma affected health in the past } 12 \text { months"; }\end{array}$} \\
\hline \multicolumn{2}{|l|}{ Code } \\
\hline & PENDING as of $1 / 31 / 07$ \\
\hline
\end{tabular}




\begin{tabular}{|c|c|}
\hline INDICATOR 1.11 & $\begin{array}{l}\text { Overall, would you say (child's name)'s asthma puts a burden on your } \\
\text { family a great deal, a medium amount, a little, or not at all? }\end{array}$ \\
\hline Survey Items Used & S2Q51 \\
\hline Numerators & $\begin{array}{l}\text { - Children with asthma whose families are affected by it a great deal or } \\
\text { medium amount } \\
\text { - Children with asthma whose families are affected by it a little or not at all }\end{array}$ \\
\hline Denominator & Children/youth ages 0-17 years whose parents report child still has asthma \\
\hline Description & $\begin{array}{l}\text { Among children/youth ages } 0-17 \text { with asthma, percent whose parents report } \\
\text { that the child's asthma affects the family a great deal or medium amount, or } \\
\text { that it affects the family a little or not at all. }\end{array}$ \\
\hline \multicolumn{2}{|l|}{ Notes for Data-Users } \\
\hline \multicolumn{2}{|c|}{$\begin{array}{l}\text { The question "Does child still have asthma?" (S2Q49) was only asked for children whose parents } \\
\text { reported ever being told that the child had asthma (S2Q19 = Yes). Only those parents who reported the } \\
\text { child still has asthma (S2Q49 = Yes) were asked questions about its effects on the child and family. }\end{array}$} \\
\hline \multicolumn{2}{|c|}{ SAS Code and Annotation } \\
\hline \multicolumn{2}{|l|}{ Format } \\
\hline \multicolumn{2}{|c|}{$\begin{array}{l}\text { value affects } / * i n d 1 \_11^{*} / \\
\mathbf{1}=\text { "Affects family a great deal or medium amount" } \\
\mathbf{2}=\text { "Affects family a little or not at all"; }\end{array}$} \\
\hline \multicolumn{2}{|l|}{ Code } \\
\hline \multicolumn{2}{|c|}{$\begin{array}{l}\text { ind1_11 = ; } \\
\text { if s2q51 in }(\mathbf{1}, \mathbf{2}) \text { then ind1_11 = } \mathbf{1} ; \\
\text { if s2q51 in }(\mathbf{3}, \mathbf{4}) \text { then ind1_11 = } \mathbf{2} ; \\
\text { if s2q51 in }(\mathbf{6}, \mathbf{7}) \text { then ind1_11 = ; } \\
\text { label ind1_11 = "Indicator } 1.11 \text { : Overall, would you say (child's name)'s asthma puts a burden on your } \\
\text { family a great deal, a medium amount, a little, or not at all? (S2Q51 - only asked for children who } \\
\text { currently have asthma)"; }\end{array}$} \\
\hline
\end{tabular}




\begin{tabular}{|c|c|}
\hline INDICATOR 1.12 & $\begin{array}{l}\text { During the past } 12 \text { months, has (child's name) stayed overnight in a } \\
\text { hospital because of his/her asthma? }\end{array}$ \\
\hline Survey Items Used & S2Q19, S2Q53 \\
\hline Numerator & $\begin{array}{l}\text { Children/youth who ever had asthma and were hospitalized overnight for } \\
\text { asthma during past } 12 \text { months }\end{array}$ \\
\hline Denominator & Children/youth ages 0-17 years \\
\hline Description & $\begin{array}{l}\text { Percent of children/youth ages } 0-17 \text { whose parents report that the child has } \\
\text { ever had asthma and stayed overnight in a hospital for asthma during the past } \\
12 \text { months. }\end{array}$ \\
\hline \multicolumn{2}{|l|}{ Notes for Data-Users } \\
\hline \multicolumn{2}{|c|}{$\begin{array}{l}\text { S2Q53 was only asked for children whose parents reported ever being told that the child had asthma } \\
\text { (S2Q19 = Yes). }\end{array}$} \\
\hline \multicolumn{2}{|c|}{ SAS Code and Annotation } \\
\hline \multicolumn{2}{|l|}{ Format } \\
\hline \multicolumn{2}{|c|}{$\begin{array}{l}\text { value asthma /*ind1_12*/ } \\
\mathbf{1}=\text { "Have asthma ever, no hospitalizations" } \\
\mathbf{2}=\text { "Have asthma ever, yes hospitalized for asthma" } \\
\mathbf{3}=\text { "Do not have asthma"; }\end{array}$} \\
\hline \multicolumn{2}{|l|}{ Code } \\
\hline \multicolumn{2}{|c|}{$\begin{array}{l}\text { ind1_12 = ; } \\
\text { if s2q19 eq } \mathbf{1} \text { then do ind1_12 = s2q53; end; } \\
\text { if s2q19 eq } \mathbf{0} \text { and s2q55 eq . then ind1_12 = 3; } \\
\text { if ind1_12 in }(\mathbf{0}, \mathbf{1}) \text { then do ind1_12 = ind1_12 + } \mathbf{1} \text {; end; } \\
\text { if ind1_12 in }(\mathbf{6}, \mathbf{7}) \text { then ind1_12 = ; } \\
\text { label ind1_12 = "Indicator } 1.12: \text { During the past } 12 \text { months, has (child's name) stayed overnight in a } \\
\text { hospital because of his/her asthma? (derived_national-level data only)"; }\end{array}$} \\
\hline
\end{tabular}




\begin{tabular}{|c|c|}
\hline INDICATOR 2.1 & $\begin{array}{l}\text { How many children have parents with one or more concerns about child's } \\
\text { learning, development, or behavior? }\end{array}$ \\
\hline Survey Items Used & S6Q08; S6Q09 - S6Q27 \\
\hline Numerator & $\begin{array}{l}\text { Children whose parents report one or more concerns about child's learning, } \\
\text { development, or behavior (S6Q08 = Yes and/or any of S6Q09 - S6Q27 = "a } \\
\text { little" or "a lot") }\end{array}$ \\
\hline Denominator & Children ages $0-5$ years \\
\hline Description & $\begin{array}{l}\text { Percent of children ages 0-5 with parents who reported one or more concerns } \\
\text { about their learning, development, or behavior (S6Q08 = Yes and/or any of } \\
\text { S6Q09-S6Q27 = "a little" or "a lot"). }\end{array}$ \\
\hline \multicolumn{2}{|l|}{ Notes for Data-Users } \\
\hline \multicolumn{2}{|c|}{$\begin{array}{l}\text { Question S6Q08 is asked for all children ages } 0-5 \text { years. Age appropriate subsets of questions S6Q09 to } \\
\text { S6Q27 are asked for children ages } 4 \text { months through } 5 \text { years. Items S6Q08 - S6Q27 are from the } \\
\text { Parents' Evaluation of Developmental Status (PEDS), a parent-reported screening instrument for } \\
\text { identifying young children at risk for developmental delay. The PEDS is a standardized tool designed to } \\
\text { identify children at risk for developmental, behavioral, or social delays. For information about the } \\
\text { development and scoring of the PEDS, visit http://www.pedstest.com @ } 2005 \text {, Frances Page Glascoe, } \\
\text { Parents' Evaluation of Developmental Status - Survey Version. Forepath.org, PO Box 23186, } \\
\text { Washington, DC } 20026 \text {. }\end{array}$} \\
\hline \multicolumn{2}{|c|}{$\begin{array}{l}\text { The NSCH uses the non-clinical version of the PEDS. Researchers interested in analyzing NSCH data } \\
\text { collected with the PEDS instrument will need to consult PEDS documentation for scoring instructions at } \\
\text { www.pedstest.com. Health care providers interested in using the PEDS to assess or make decisions } \\
\text { about the developmental status for individual children, need to use the clinical version of the PEDS } \\
\text { instrument, which can be obtained from Ellsworth \& Vandermeer Press, LLC. }\end{array}$} \\
\hline \multicolumn{2}{|c|}{$\begin{array}{l}\text { National data for the PEDS are also available from the } 2000 \text { National Survey of Early Childhood Health. } \\
\text { See www.cdc.gov/nchs/data/slaits/NSCH_Methodology_Report.pdf for more information. }\end{array}$} \\
\hline \multicolumn{2}{|c|}{ SAS Code and Annotation } \\
\hline \multicolumn{2}{|l|}{ Format } \\
\hline \multicolumn{2}{|c|}{$\begin{array}{l}\text { value concern } / * \text { ind2_1*/ } \\
\qquad \begin{array}{l}\mathbf{1}=\text { "1 or more parent-reported concerns" } \\
\mathbf{2}=\text { "No parent-reported concerns"; }\end{array}\end{array}$} \\
\hline
\end{tabular}




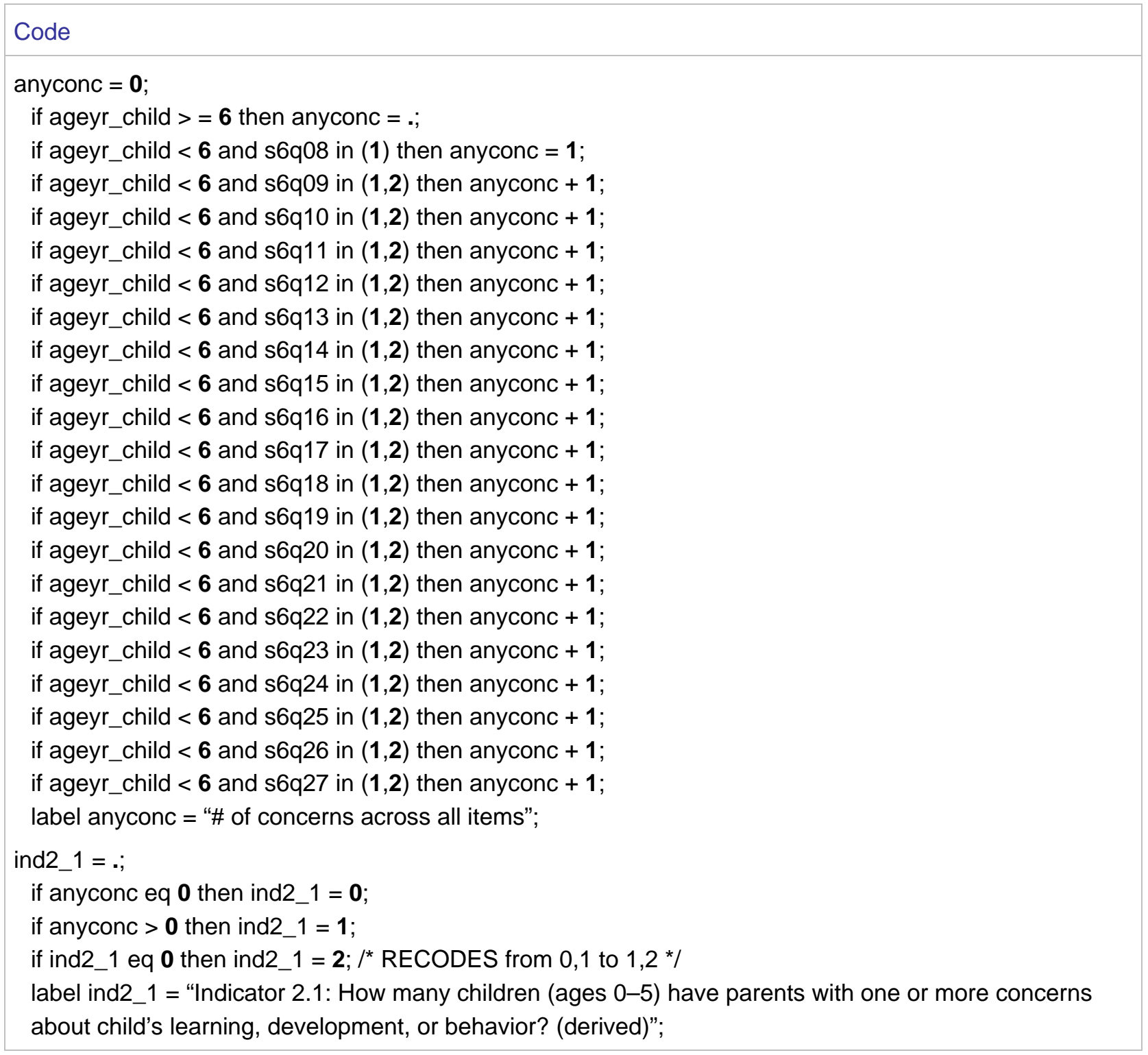




\begin{tabular}{|c|c|}
\hline INDICATOR 2.2 & How many children are at risk for developmental delay? \\
\hline Survey Items Used & S6Q08; S6Q13 - S6Q27 \\
\hline Numerator & $\begin{array}{l}\text { Young children with one or more parent-reported concerns indicative of } \\
\text { developmental risk }\end{array}$ \\
\hline Denominator & Children ages $1-5$ years \\
\hline Description & $\begin{array}{l}\text { Percent of children ages } 1-5 \text { with one or more parent-reported concerns } \\
\text { indicative of developmental risk (S6Q13 - S6Q27, response of "a little" or "a lot" } \\
\text { to one or more). }\end{array}$ \\
\hline \multicolumn{2}{|l|}{ Notes for Data-Users } \\
\hline \multicolumn{2}{|c|}{$\begin{array}{l}\text { Items S6Q08 - S6Q27 are from the Parents' Evaluation of Developmental Status (PEDS), a parent- } \\
\text { reported screening instrument for identifying young children at risk for developmental delay. The PEDS is } \\
\text { a standardized tool designed to identify children at risk for developmental, behavioral, or social delays. For } \\
\text { information about the development and scoring of the PEDS, visit http://www.pedstest.com, (C2005, } \\
\text { Frances Page Glascoe, Parents' Evaluation of Developmental Status-Survey Version. Forepath.org, PO } \\
\text { Box 23186, Washington, DC } 20026 \text {. }\end{array}$} \\
\hline \multicolumn{2}{|c|}{$\begin{array}{l}\text { The NSCH uses the non-clinical version of the PEDS. Researchers interested in analyzing NSCH data } \\
\text { collected with the PEDS instrument will need to consult PEDS documentation for scoring instructions at } \\
\text { www.pedstest.com. Health care providers interested in using the PEDS to assess or make decisions } \\
\text { about the developmental status for individual children, need to use the clinical version of the PEDS } \\
\text { instrument, which can be obtained from Ellsworth \& Vandermeer Press, LLC. }\end{array}$} \\
\hline \multicolumn{2}{|c|}{$\begin{array}{l}\text { National data for the PEDS are also available from the } 2000 \text { National Survey of Early Childhood Health. } \\
\text { See www.cdc.gov/nchs/data/slaits/NSCH_Methodology_Report.pdf for more information. }\end{array}$} \\
\hline \multicolumn{2}{|c|}{ SAS Code and Annotation } \\
\hline \multicolumn{2}{|l|}{ Format } \\
\hline
\end{tabular}




\begin{tabular}{|c|c|}
\hline INDICATOR 2.3 & $\begin{array}{l}\text { How many children/youth have moderate or severe difficulties in the areas } \\
\text { of emotions, concentration, behavior, or being able to get along with other } \\
\text { people? }\end{array}$ \\
\hline Survey Items Used & S2Q59 and S2Q60 \\
\hline Numerator & $\begin{array}{l}\text { Children/youth with socio-emotional difficulties described as moderate or severe } \\
\text { by their parents }\end{array}$ \\
\hline Denominator & Children/youth ages 3-17 years \\
\hline Description & $\begin{array}{l}\text { Among all children/youth ages 3-17, percent with moderate to severe socio- } \\
\text { emotional difficulties, as reported by their parents (S2Q60 = "Moderate" or } \\
\text { "Severe"). }\end{array}$ \\
\hline \multicolumn{2}{|l|}{ Notes for Data-Users } \\
\hline \multicolumn{2}{|c|}{$\begin{array}{l}\text { Questions S2Q59 and S2Q60 were drawn from the impact supplement to the Strengths and Difficulties } \\
\text { Questionnaire (SDQ). Due to an inadvertent error in the questionnaire development process, the answer } \\
\text { choices for the follow-up question do not match the answer choices for the copyrighted SDQ. Analysts } \\
\text { should use caution when comparing estimates derived from the NSCH follow-up question to estimates } \\
\text { derived from the proper SDQ impact question used in other surveys (e.g., the NHIS). See } \\
\text { www.cdc.gov/nchs/data/slaits/NSCH_Methodology_Report.pdf for more information. }\end{array}$} \\
\hline \multicolumn{2}{|c|}{ SAS Code and Annotation } \\
\hline \multicolumn{2}{|l|}{ Format } \\
\hline \multicolumn{2}{|c|}{$\begin{array}{l}\text { value diff } / * \text { ind2_ } 3 * / \\
\mathbf{1}=\text { "Moderate or severe difficulties in } 1 \text { or more areas" } \\
\mathbf{2}=\text { "No difficulties or minor difficulties"; }\end{array}$} \\
\hline \multicolumn{2}{|l|}{ Code } \\
\hline \multicolumn{2}{|c|}{ 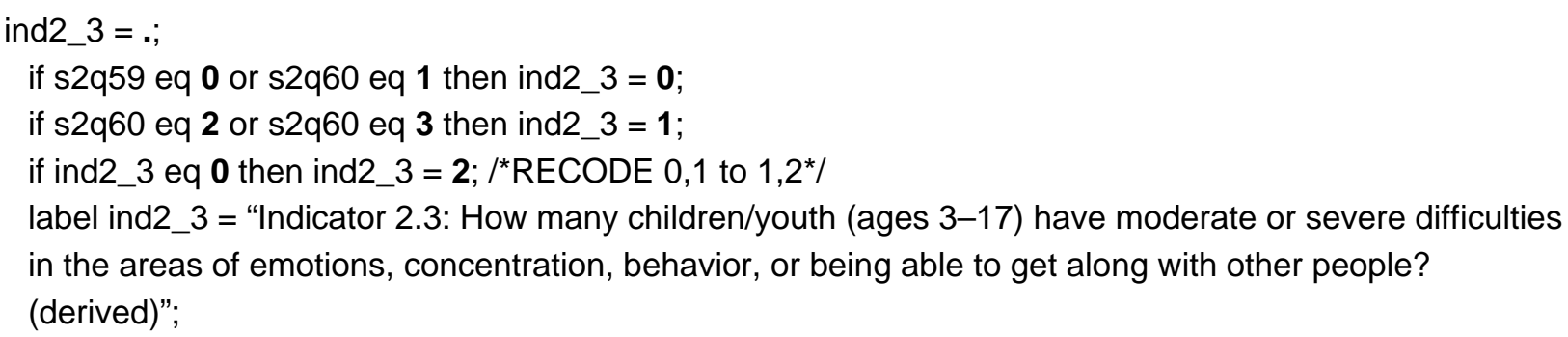 } \\
\hline
\end{tabular}




\begin{tabular}{|c|c|}
\hline INDICATOR 2.4 & $\begin{array}{l}\text { Overall, would you say (child's name)'s mental and emotional health puts a } \\
\text { burden on your family a great deal, a medium amount, a little, or not at all? }\end{array}$ \\
\hline Survey Items Used & S2Q61 \\
\hline Numerator & $\begin{array}{l}\text { Children/youth with socio-emotional difficulties whose families report being } \\
\text { affected a great deal/medium amount }\end{array}$ \\
\hline Denominator & $\begin{array}{l}\text { Children/youth ages 3-17 years who have one or more parent-reported } \\
\text { difficulties in the socio-emotional area }\end{array}$ \\
\hline Description & $\begin{array}{l}\text { Among children/youth ages 3-17 who have one or more parent-reported socio- } \\
\text { emotional difficulties (S2Q59 = "Yes"), percent whose families are affected more } \\
\text { than a little (S2Q61 = "medium" or "a great deal"). }\end{array}$ \\
\hline \multicolumn{2}{|l|}{ Notes for Data-Users } \\
\hline \multicolumn{2}{|c|}{$\begin{array}{l}\text { Only parents report who reported that their children has one or more socio-emotional difficulties (S } \\
\text { Yes) were asked questions about how these difficulties affect the child and the child's family. }\end{array}$} \\
\hline \multicolumn{2}{|c|}{ SAS Code and Annotation } \\
\hline \multicolumn{2}{|l|}{ Format } \\
\hline \multicolumn{2}{|c|}{$\begin{array}{l}\text { value affects } / * \text { ind1_11 and ind2_4*/ } \\
\mathbf{1}=\text { = "Affects family a great deal or medium amount" } \\
\mathbf{2}=\text { "Affects family a little or not at all"; }\end{array}$} \\
\hline \multicolumn{2}{|l|}{ Code } \\
\hline \multicolumn{2}{|c|}{ 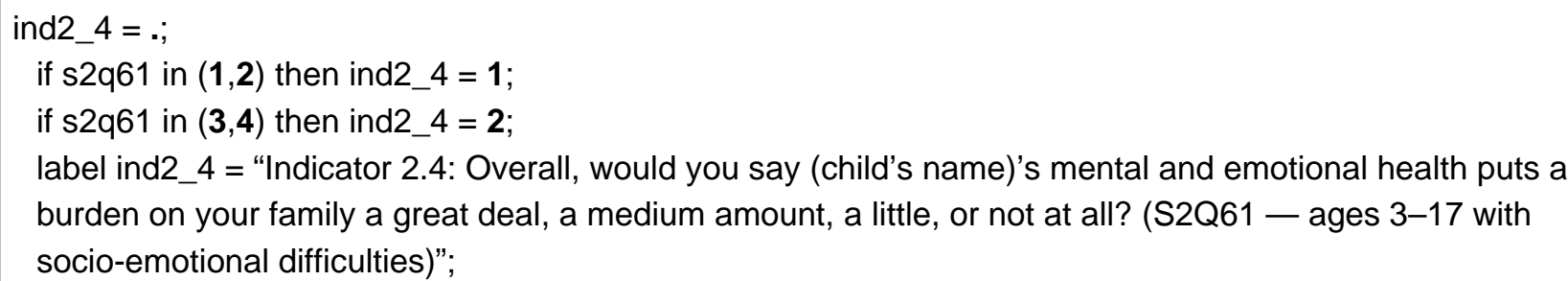 } \\
\hline
\end{tabular}




\begin{tabular}{|c|c|}
\hline INDICATOR 2.5 & $\begin{array}{l}\text { How many children/youth often exhibit caring, respectful behaviors when } \\
\text { interacting other children and adults? }\end{array}$ \\
\hline Survey Items Used & S7Q52, S7Q53, S7Q54, S7Q59 \\
\hline Numerator & In development \\
\hline Denominator & In development \\
\hline \multicolumn{2}{|l|}{ Description } \\
\hline \multicolumn{2}{|l|}{ Notes for Data-Users } \\
\hline \multicolumn{2}{|c|}{$\begin{array}{l}\text { This item is currently in development. For results on the individual items included in this indicator, please } \\
\text { check individual questions in Section } 7 \text { of the survey: S7Q52, S7Q53, S7Q54, S7Q59. }\end{array}$} \\
\hline \multicolumn{2}{|c|}{$\begin{array}{l}\text { S7Q52 was drawn from the Positive Behaviors Scale. For more information, email SLAITS staff at } \\
\text { slaits@cdc.gov or visit www.cdc.gov/nchs/data/slaits/NSCH_Methodology_Report.pdf }\end{array}$} \\
\hline \multicolumn{2}{|c|}{ SAS Code and Annotation } \\
\hline \multicolumn{2}{|l|}{ Format } \\
\hline \multicolumn{2}{|l|}{ Code } \\
\hline & In development as of $1 / 31 / 07$ \\
\hline
\end{tabular}




\section{INDICATOR 2.6}

Survey Items Used

Numerator

Denominator

Description
S7Q41, S7Q44, S7Q45, S7Q56

Children/youth who often exhibit two or more problematic behaviors

Children/youth ages 6-17 years

Percent of children/youth ages 6-17 who often exhibit two or more problematic behaviors (S7Q41, S7Q44, S7Q45, S7Q56; response of "Usually/always" to "two or more").

\section{Notes for Data-Users}

Problematic behaviors are defined as arguing too much, bullying or being cruel to others, disobeying, and acting stubborn, sullen or irritable.

Questions S7Q41, S7Q44, S7Q45, S7Q48, S7Q56, S7Q62, and S7Q63 were drawn from the Behavior Problems Index. For more information, email SLAITS staff at slaits@cdc.gov or visit www.cdc.gov/nchs/data/slaits/NSCH_Methodology_Report.pdf.

\section{SAS Code and Annotation}

\section{Format}

value probbeh $/ *$ ind2_6*/

$\mathbf{1}=$ "Often exhibits 2 or more problematic behaviors"

2 = "Seldom exhibits problematic behaviors";

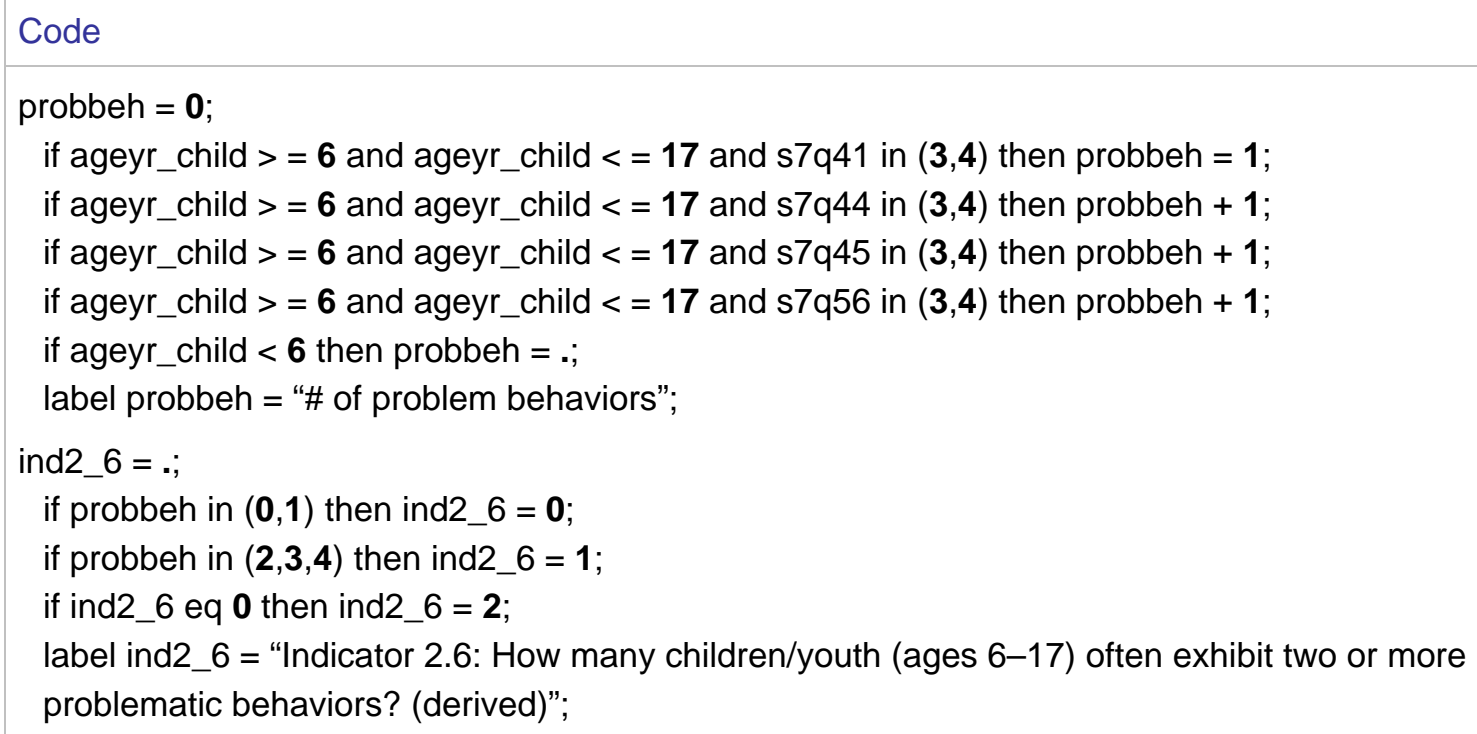




\begin{tabular}{|c|c|}
\hline INDICATOR 2.7 & $\begin{array}{l}\text { How many children/youth whose parents have ever been told child has } \\
\text { ADD/ADHD currently take medication for this condition? }\end{array}$ \\
\hline Survey Items Used & S2Q21 and S2Q62 \\
\hline Numerator & Children/youth who are currently taking medication for ADD/ADHD \\
\hline Denominator & Children/youth ages $2-17$ years \\
\hline Description & $\begin{array}{l}\text { Among children/youth ages } 2-17 \text {, percent whose parents reported having ever } \\
\text { been told by a doctor that child has ADD/ADHD and who are currently taking } \\
\text { medication for this condition (S2Q21 and S2Q62 = "Yes"). }\end{array}$ \\
\hline \multicolumn{2}{|l|}{ Notes for Data-Users } \\
\hline \multicolumn{2}{|c|}{$\begin{array}{l}\text { "ADD/ADHD Ever" is defined as children whose parents report ever being told by a doctor or health } \\
\text { professional that child has Attention Deficit Disorder (ADD) or Attention Deficit Hyperactivity Disorder } \\
(A D H D)(S 2 Q 21=\text { Yes). }\end{array}$} \\
\hline \multicolumn{2}{|c|}{$\begin{array}{l}\text { Use caution when interpreting responses to the questions about health conditions in the NSCH. A } \\
\text { response of YES to having "ever been told by a doctor or other health professional" that a child has a } \\
\text { specific health condition DOES NOT necessarily indicate that the child still has or ever had that particular } \\
\text { condition. For this reason, affirmative responses to the specific health condition questions should not be } \\
\text { interpreted as current prevalence. }\end{array}$} \\
\hline \multicolumn{2}{|c|}{ SAS Code and Annotation } \\
\hline \multicolumn{2}{|l|}{ Format } \\
\hline \multicolumn{2}{|c|}{$\begin{array}{l}\mathbf{1}=\text { "ADD/ADHD ever; child currently takes medication" } \\
\mathbf{2}=\text { "ADD/ADHD ever; child not taking medication now" } \\
\mathbf{3}=\text { "Never told child has ADD/ADHD"; }\end{array}$} \\
\hline \multicolumn{2}{|l|}{ Code } \\
\hline \multicolumn{2}{|c|}{$\begin{array}{l}\text { ind2_7 = s2q62; } \\
\text { if s2q62 in }(\mathbf{6}, 7) \text { then ind2_7 = ; } \\
\text { if s2q21 eq } 0 \text { then ind2_7 = 3; } \\
\text { if ind2_7 eq } 0 \text { then ind2_7 = 2; /*RECODE } 0,1,3 \text { into } 1,2,3^{\star} / \\
\text { label ind2_7 = "Indicator } 2.7 \text { : How many children/youth (ages } 2-17) \text { whose parents have ever been told } \\
\text { child has ADD/ADHD currently take medication for this condition?"; }\end{array}$} \\
\hline
\end{tabular}




\begin{tabular}{|c|c|}
\hline INDICATOR 2.8 & $\begin{array}{l}\text { Has a doctor or health professional ever told you that (child's name) has } \\
\text { autism? }\end{array}$ \\
\hline Survey Items Used & S2Q35 \\
\hline Numerator & $\begin{array}{l}\text { Children/youth whose parents report ever being told by a doctor/health } \\
\text { professional that child has autism. }\end{array}$ \\
\hline Denominator & Children/youth ages 0-17 years \\
\hline Description & $\begin{array}{l}\text { Percent of children/youth ages } 0-17 \text { whose parents reported ever being told by a } \\
\text { doctor/health professional that child had autism }(\mathrm{S} 2 \mathrm{Q} 35=\mathrm{Yes}) .\end{array}$ \\
\hline \multicolumn{2}{|l|}{ Notes for Data-Users } \\
\hline \multicolumn{2}{|c|}{$\begin{array}{l}\text { Use caution when interpreting responses to the questions about specific health conditions in the NSCH. A } \\
\text { response of YES to "having ever been told by a doctor or other health professional" that a child has a } \\
\text { specific condition DOES NOT necessarily indicate that the child still has or ever had that particular } \\
\text { condition. For this reason, affirmative responses to the specific health condition questions should not be } \\
\text { interpreted as current prevalence. }\end{array}$} \\
\hline \multicolumn{2}{|c|}{ SAS Code and Annotation } \\
\hline \multicolumn{2}{|l|}{ Format } \\
\hline \multicolumn{2}{|c|}{$\begin{array}{l}\text { value yn /*ind1_3 ind1_7 ind1_8 ind2_8 ind3_1 ind4_6 ind4_9 ind5_2 ind6_14*/ } \\
\mathbf{1}=\text { "Yes" } \\
\mathbf{2}=\text { "No"; }\end{array}$} \\
\hline \multicolumn{2}{|l|}{ Code } \\
\hline \multicolumn{2}{|c|}{ 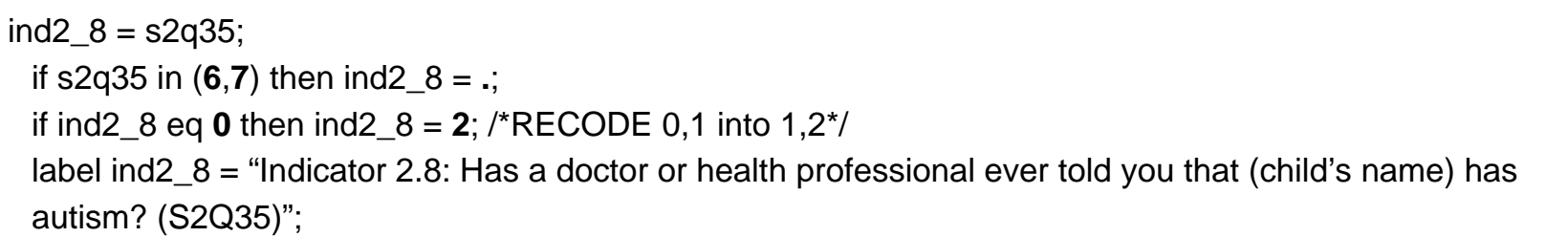 } \\
\hline
\end{tabular}




\begin{tabular}{|c|c|}
\hline INDICATOR 3.1 & $\begin{array}{l}\text { Does (child's name) have any kind of health care coverage, including } \\
\text { health insurance, prepaid plans such as HMOs, or government plans such } \\
\text { as Medicaid? }\end{array}$ \\
\hline Survey Items Used & S3Q01 \\
\hline Numerator & Children/youth who currently have health care coverage \\
\hline Denominator & Children/youth ages 0-17 years \\
\hline Description & $\begin{array}{l}\text { Percent of children/youth ages } 0-17 \text { with any kind of health care coverage, } \\
\text { including health insurance, prepaid plans such as HMOs, or government plans } \\
\text { such as Medicaid (S3Q01 = Yes). }\end{array}$ \\
\hline \multicolumn{2}{|l|}{ Notes for Data-Users } \\
\hline \multicolumn{2}{|c|}{ SAS Code and Annotation } \\
\hline \multicolumn{2}{|l|}{ Format } \\
\hline \multicolumn{2}{|c|}{$\begin{array}{l}\text { value yn /*ind1_3 ind1_7 ind1_8 ind2_8 ind3_1 ind4_6 ind4_9 ind5_2 ind6_14*/ } \\
\mathbf{1}=\text { = "Yes" } \\
\mathbf{2}=\text { "No"; }\end{array}$} \\
\hline \multicolumn{2}{|l|}{ Code } \\
\hline \multicolumn{2}{|c|}{$\begin{array}{l}\text { ind3_1 = ; } \\
\text { if s3q01 eq } 1 \text { then ind3_1 = 1; /*Insured*/ } \\
\text { if s3q01 = } 0 \text { then ind3_1 = 2; /*Not Insured*/ } \\
\text { label ind3_1 = "Indicator 3.1: Does (child's name) have any kind of health care coverage, including } \\
\text { health insurance, prepaid plans such as HMOs, or government plans such as Medicaid? (S3Q01)"; }\end{array}$} \\
\hline
\end{tabular}




\begin{tabular}{|c|c|}
\hline INDICATOR 3.2 & $\begin{array}{l}\text { How many children/youth had consistent health insurance coverage } \\
\text { during the past } 12 \text { months? }\end{array}$ \\
\hline Survey Items Used & S3Q01 and S3Q04 \\
\hline Numerator & Children/youth who were consistently insured during the past year \\
\hline Denominator & Children/youth ages 0-17 years \\
\hline Description & $\begin{array}{l}\text { Children/youth who were insured at the time of the survey }(\mathrm{S} 3 \mathrm{Q} 01=\mathrm{Yes}) \text { and } \\
\text { who did not experience periods of no insurance during the past } 12 \text { months } \\
(\mathrm{S} 3 \mathrm{Q} 04=\mathrm{No}) \text {. }\end{array}$ \\
\hline \multicolumn{2}{|l|}{ Notes for Data-Users } \\
\hline \multicolumn{2}{|c|}{ SAS Code and Annotation } \\
\hline \multicolumn{2}{|l|}{ Format } \\
\hline \multicolumn{2}{|c|}{$\begin{array}{l}\text { value cons_ins /*ind3_2*/ } \\
\mathbf{1}=\text { = "Currently uninsured or periods of no coverage during year" } \\
\mathbf{2}=\text { "Consistently insured during past year"; }\end{array}$} \\
\hline \multicolumn{2}{|l|}{ Code } \\
\hline \multicolumn{2}{|c|}{$\begin{array}{l}\text { ind3_2 =; } \\
\text { if S3Q01 = } 0 \text { or S3Q04 = } \mathbf{1} \text { then ind3_2 = 1; } \\
\text { if S3Q04 = } 0 \text { then ind3_2 = 2; } \\
\text { label ind3_2 = "Indicator 3.2: How many children/youth (ages 0-17) had consistent health insurance } \\
\text { coverage during the past } 12 \text { months? (derived)"; }\end{array}$} \\
\hline
\end{tabular}




\begin{tabular}{|c|c|}
\hline INDICATOR 3.3 & $\begin{array}{l}\text { What type of health insurance coverage, if any, did children/youth have at } \\
\text { the time of the survey? }\end{array}$ \\
\hline Survey Items Used & S3Q01, S3Q02 \\
\hline Numerators & $\begin{array}{l}\text { - } \quad \text { Children/youth who are currently uninsured } \\
\text { - } \quad \text { Children/youth who currently have private insurance coverage } \\
\text { - } \quad \text { Children/youth who currently have public insurance coverage }\end{array}$ \\
\hline Denominator & Children/youth ages 0-17 years \\
\hline Description & $\begin{array}{l}\text { Children/youth ages } 0-17 \text { whose health insurance coverage at the time of the } \\
\text { survey was either private }(\mathrm{S} 3 \mathrm{Q} 01=\text { Yes and S3Q02 = No) or public }(\mathrm{S} 3 \mathrm{Q} 01 \\
\text { and S3Q02 = Yes) or none }(\mathrm{S} 3 \mathrm{Q} 01=\mathrm{No}) .\end{array}$ \\
\hline \multicolumn{2}{|l|}{ Notes for Data-Users } \\
\hline \multicolumn{2}{|c|}{$\begin{array}{l}\text { Private health insurance is defined as any type of health insurance } \\
\text { programs. Public health insurance is defined as Medicaid or the St } \\
\text { Program (SCHIP). }\end{array}$} \\
\hline \multicolumn{2}{|c|}{ SAS Code and Annotation } \\
\hline \multicolumn{2}{|l|}{ Format } \\
\hline \multicolumn{2}{|c|}{$\begin{array}{l}\text { value insure } / * i n d 3 \_1 * / \\
\mathbf{1}=\text { "Public insurance" } \\
\mathbf{2}=\text { "Private insurance" } \\
\mathbf{3}=\text { "Uninsured"; }\end{array}$} \\
\hline \multicolumn{2}{|l|}{ Code } \\
\hline \multicolumn{2}{|c|}{ 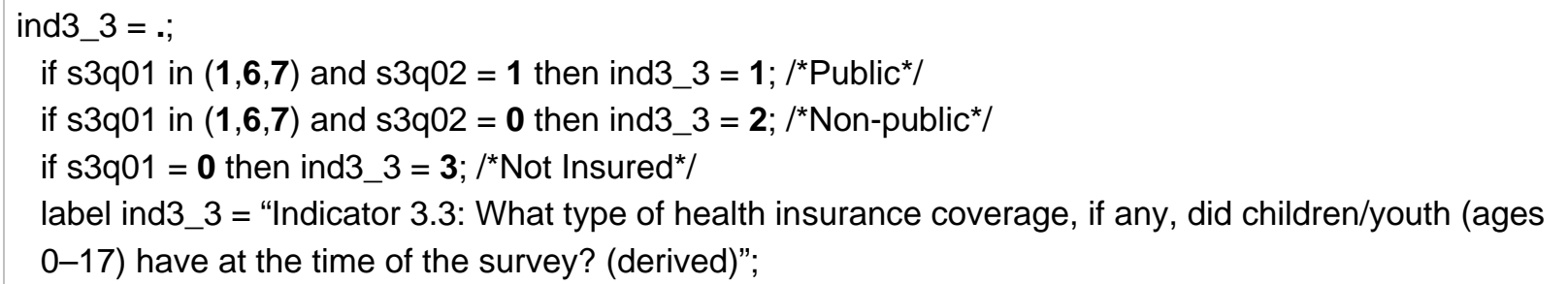 } \\
\hline
\end{tabular}




\begin{tabular}{|c|c|}
\hline INDICATOR 4.1 & $\begin{array}{l}\text { How many children/youth had one or more preventive medical care } \\
\text { visits during the past } 12 \text { months? }\end{array}$ \\
\hline Survey Items Used & S4Q01 and S4Q03 \\
\hline Numerator & Children/youth who had one or more preventive medical care visits \\
\hline Denominator & Children/youth ages 0-17 years \\
\hline Description & $\begin{array}{l}\text { Percent of children/youth ages } 0-17 \text { with one or more preventive medical } \\
\text { care visits in the past } 12 \text { months. }\end{array}$ \\
\hline \multicolumn{2}{|l|}{ Notes for Data-Users } \\
\hline \multicolumn{2}{|c|}{$\begin{array}{l}\text { Denominator includes children/youth who did not see a health care provider for any type of medical care } \\
\text { during } 12 \text { past months }(\mathrm{S} 4 \mathrm{Q} 01=\mathrm{No}) \text {. }\end{array}$} \\
\hline \multicolumn{2}{|c|}{ SAS Code and Annotation } \\
\hline \multicolumn{2}{|l|}{ Format } \\
\hline \multicolumn{2}{|c|}{$\begin{array}{l}\text { value prev_med /*ind4_1*/ } \\
\mathbf{1}=\text { "One or more preventive medical care visits" } \\
\mathbf{2}=\text { "NO preventive medical care visits"; }\end{array}$} \\
\hline \multicolumn{2}{|l|}{ Code } \\
\hline \multicolumn{2}{|c|}{ 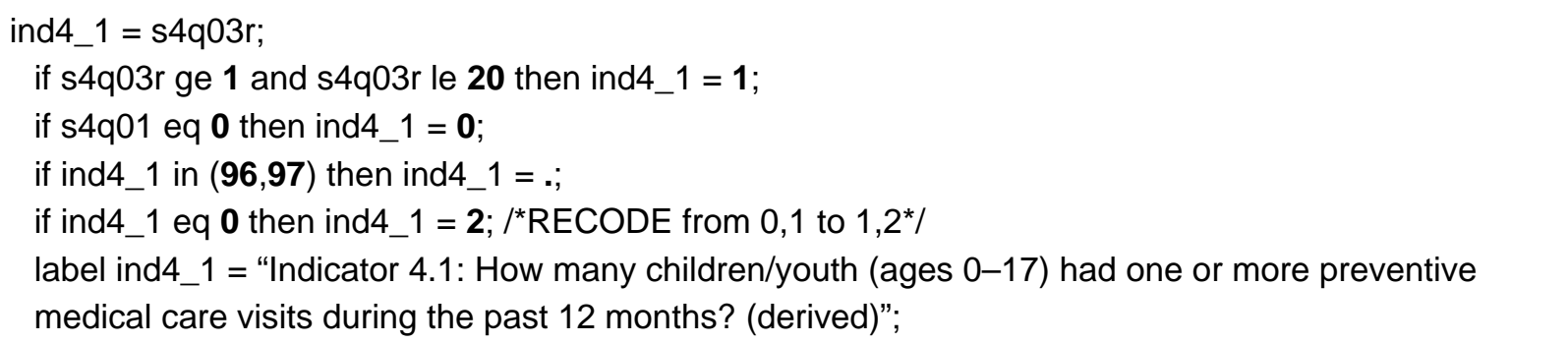 } \\
\hline
\end{tabular}




\begin{tabular}{|c|c|}
\hline INDICATOR 4.2 & $\begin{array}{l}\text { How many children/youth with a need for routine preventive dental care, } \\
\text { such as check-ups, screenings, and sealants, got all the care that was } \\
\text { needed? }\end{array}$ \\
\hline Survey Items Used & S4Q10 and S4Q13 \\
\hline Numerator & Children/youth who got all needed preventive dental care \\
\hline Denominator & $\begin{array}{l}\text { Children/youth ages } 1-17 \text { years who needed preventive dental care during } \\
\text { past } 12 \text { months }\end{array}$ \\
\hline Description & $\begin{array}{l}\text { Percent of children/youth ages } 1-17 \text { who received needed preventive dental } \\
\text { care during the past } 12 \text { months. }\end{array}$ \\
\hline \multicolumn{2}{|l|}{ Notes for Data-Users } \\
\hline \multicolumn{2}{|c|}{$\begin{array}{l}\text { Children under } 1 \text { and children/youth who did not have natural teeth at the time of the survey (S2Q54 = } \\
\text { has no natural teeth) were not included in any of the indicators addressing dental care. Children/youth } \\
\text { who "needed preventive dental care" are defined as children/youth who saw a dentist during the past } 12 \\
\text { months but did not get preventive dental care (S4Q09 = Yes) OR children/youth who needed preventive } \\
\text { dental care during the past } 12 \text { months even though they did not see a dentist (S4Q10 = Yes). }\end{array}$} \\
\hline \multicolumn{2}{|c|}{ SAS Code and Annotation } \\
\hline \multicolumn{2}{|l|}{ Format } \\
\hline \multicolumn{2}{|c|}{$\begin{array}{l}\text { value prev_den } / * i n d 4 \_2^{\star} / \\
\mathbf{1}=\text { "Did not get all needed care" } \\
\mathbf{2}=\text { "Got all needed care"; }\end{array}$} \\
\hline \multicolumn{2}{|l|}{ Code } \\
\hline \multicolumn{2}{|c|}{$\begin{array}{l}\text { ind4_2 = ; } \\
\text { if } \mathrm{s} 4 \mathrm{Q} 13=\mathbf{0} \text { or } \mathrm{s} 4 \mathrm{Q} 10=\mathbf{1} \text { then ind4_2 = } \mathbf{1} \text {; } \\
\text { if } \mathrm{s} 4 \mathrm{Q} 13=\mathbf{1} \text { then ind4_2 = } \mathbf{2} \\
\text { label ind4_2 = "Indicator 4.2: How many children/youth (ages } 1-17 \text { ) with a need for routine preventive } \\
\text { dental care, such as check-ups, screenings, and sealants, got all the care that was needed? } \\
\text { (derived)"; }\end{array}$} \\
\hline
\end{tabular}




\begin{tabular}{|c|c|}
\hline INDICATOR 4.3 & $\begin{array}{l}\text { How many children/youth had both medical AND dental preventive care } \\
\text { visits in the past } 12 \text { months? }\end{array}$ \\
\hline Survey Items Used & S4Q03 and S4Q09 \\
\hline Numerator & Children/youth who got both medical and dental preventive care \\
\hline Denominator & Children/youth ages 0-17 years \\
\hline Description & $\begin{array}{l}\text { Percent of children/youth ages } 0-17 \text { with both a medical AND dental } \\
\text { preventive care visit in the past } 12 \text { months. }\end{array}$ \\
\hline \multicolumn{2}{|l|}{ Notes for Data-Users } \\
\hline \multicolumn{2}{|c|}{$\begin{array}{l}\text { Children under } 12 \text { months of age needed only a preventive } m \\
\text { on this measure. }\end{array}$} \\
\hline \multicolumn{2}{|c|}{ SAS Code and Annotation } \\
\hline \multicolumn{2}{|l|}{ Format } \\
\hline \multicolumn{2}{|c|}{$\begin{array}{l}\text { value prevent } / * i n d 4 \_3^{*} / \\
\mathbf{1}=\text { "Got both medical and dental preventive care visits" } \\
\mathbf{2}=\text { "Did NOT get both medical and dental preventive care visits"; }\end{array}$} \\
\hline \multicolumn{2}{|l|}{ Code } \\
\hline \multicolumn{2}{|c|}{ 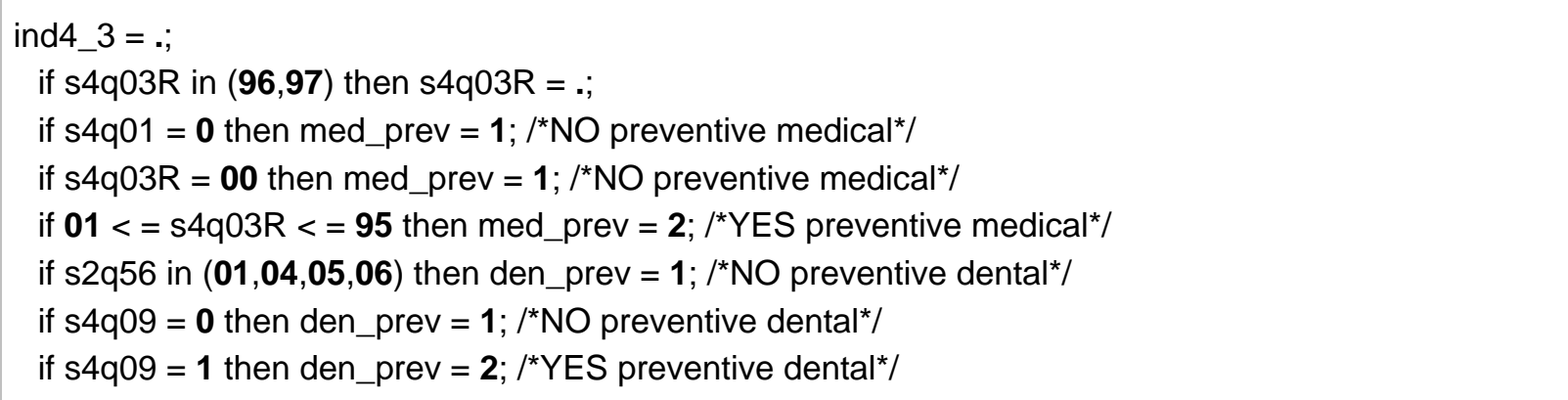 } \\
\hline \multicolumn{2}{|c|}{ 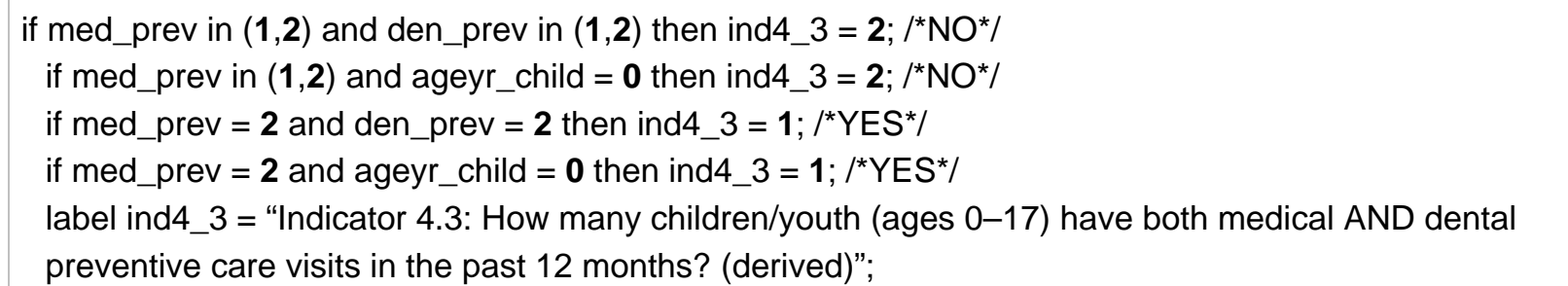 } \\
\hline
\end{tabular}




\begin{tabular}{|c|c|}
\hline INDICATOR 4.4 & $\begin{array}{l}\text { During the past } 12 \text { months, how many times did (child's name) go to a } \\
\text { hospital emergency room (ER) about his/her health? This includes ER } \\
\text { visits that resulted in a hospital admission. }\end{array}$ \\
\hline Survey Items Used & S4Q01 and S4Q04 \\
\hline Numerators & $\begin{array}{l}\text { - } \quad \text { Children/youth who did not go to a hospital ER } \\
\text { - } \quad \text { Children/youth who had one ER visit } \\
\text { - } \quad \text { Children/youth who had two or more ER visits }\end{array}$ \\
\hline Denominator & Children/youth ages 0-17 years \\
\hline Description & $\begin{array}{l}\text { Percent of children/youth ages } 0-17 \text { who went to a hospital ER about his/her } \\
\text { health no times, one time, or two or more times in the past } 12 \text { months. }\end{array}$ \\
\hline \multicolumn{2}{|l|}{ Notes for Data-Users } \\
\hline \multicolumn{2}{|c|}{$\begin{array}{l}\text { Denominator includes children who did not see a health care provider for any type of medical care } \\
\text { during } 12 \text { past months }(\mathrm{S} 4 \mathrm{Q} 01=\mathrm{No}) .\end{array}$} \\
\hline \multicolumn{2}{|c|}{ SAS Code and Annotation } \\
\hline \multicolumn{2}{|l|}{ Format } \\
\hline \multicolumn{2}{|c|}{$\begin{array}{l}\text { value emerg } / * \text { ind } 4 \_4 * / \\
\begin{array}{l}\mathbf{1}=\text { "0 times" } \\
\mathbf{2}=\text { "1 } 1 \text { time" } \\
\mathbf{3}=\text { ="2 or more times"; }\end{array}\end{array}$} \\
\hline \multicolumn{2}{|l|}{ Code } \\
\hline \multicolumn{2}{|c|}{ 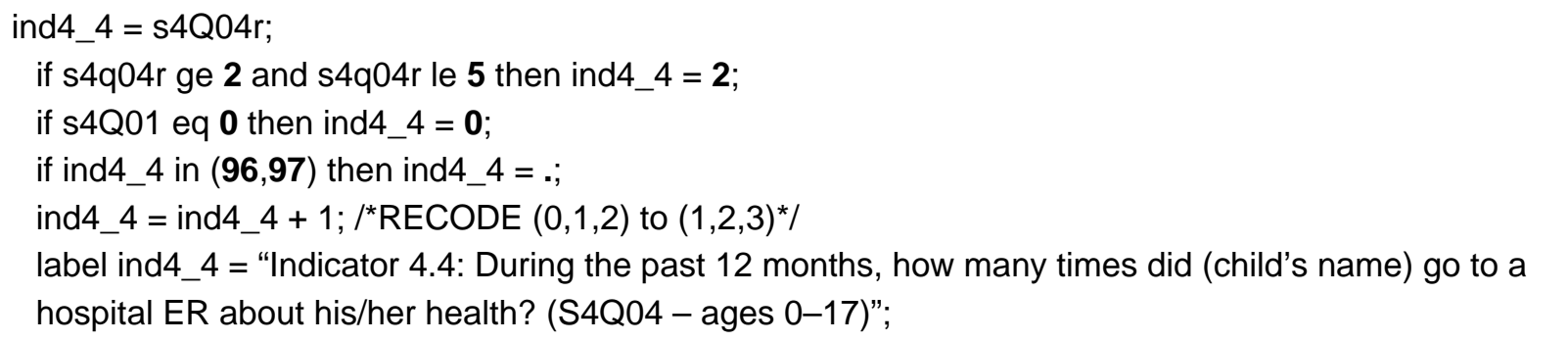 } \\
\hline
\end{tabular}




\begin{tabular}{|c|c|}
\hline INDICATOR 4.5 & $\begin{array}{l}\text { How many children/youth with current emotional, developmental, or } \\
\text { behavioral problems that require treatment or counseling received } \\
\text { some type of mental health care during the past } 12 \text { months? }\end{array}$ \\
\hline Survey Items Used & S2Q16 and S4Q23 \\
\hline Numerator & $\begin{array}{l}\text { Children/youth who received treatment or counseling for mental health } \\
\text { condition }\end{array}$ \\
\hline Denominator & $\begin{array}{l}\text { Children/youth ages 1-17 years with a current emotional, developmental, or } \\
\text { behavioral problem requiring treatment or counseling }(\mathrm{S} 2 \mathrm{Q} 16=\mathrm{Yes})\end{array}$ \\
\hline Description & $\begin{array}{l}\text { Among children/youth ages } 1-17 \text { with current emotional, developmental, or } \\
\text { behavioral problems, percent who received treatment or counseling in the } \\
\text { past } 12 \text { months. }\end{array}$ \\
\hline \multicolumn{2}{|l|}{ Notes for Data-Users } \\
\hline \multicolumn{2}{|c|}{$\begin{array}{l}\text { The denominator for this indicator only includes children/youth ages } 1-17 \text { years identified as having } \\
\text { emotional, developmental, or behavioral problems requiring treatment or counseling at the time of the } \\
\text { survey (S2Q16 = Yes). }\end{array}$} \\
\hline \multicolumn{2}{|c|}{ SAS Code and Annotation } \\
\hline \multicolumn{2}{|l|}{ Format } \\
\hline \multicolumn{2}{|c|}{$\begin{array}{l}\text { value mentcare } / * \text { ind } 4 \_5 \star / \\
\begin{array}{l}\mathbf{1}=\text { "Did NOT get mental health care/counseling" } \\
\mathbf{2}=\text { "Received mental health care/counseling of some type"; }\end{array}\end{array}$} \\
\hline \multicolumn{2}{|l|}{ Code } \\
\hline \multicolumn{2}{|c|}{$\begin{array}{l}\text { ind4_5 = .; } \\
\text { if s2Q16 eq } \mathbf{1} \text { and s4Q23 eq } \mathbf{1} \text { then ind4_5 = } \mathbf{2} \text {; } \\
\text { if s2Q16 eq } \mathbf{1} \text { and s4Q23 eq } \mathbf{0} \text { then ind4_5 = 1; } \\
\text { label ind4_5 = "Indicator } 4.5 \text { : How many children/youth (ages } 1-17 \text { ) with current emotional, } \\
\text { developmental, or behavioral problems requiring treatment or counseling received some type of mental } \\
\text { health care in the past } 12 \text { months? (derived)"; }\end{array}$} \\
\hline
\end{tabular}




\begin{tabular}{|c|c|}
\hline INDICATOR 4.6 & $\begin{array}{l}\text { During the past } 12 \text { months, did (child's name) receive all the medical } \\
\text { care he/she needed? }\end{array}$ \\
\hline Survey Items Used & S4Q07 \\
\hline Numerator & Children/youth who got all needed medical care \\
\hline Denominator & $\begin{array}{l}\text { Children/youth ages } 0-17 \text { years who needed and/or used medical care during } \\
\text { the past } 12 \text { months }\end{array}$ \\
\hline Description & $\begin{array}{l}\text { Percent of children/youth ages } 0-17 \text { received all the medical care they } \\
\text { needed in the past } 12 \text { months ( } 44007=\text { Yes). }\end{array}$ \\
\hline \multicolumn{2}{|l|}{ Notes for Data-Users } \\
\hline \multicolumn{2}{|c|}{$\begin{array}{l}\text { Children/youth who "needed and/or used medical care" are defined as those who either saw a health } \\
\text { care professional for any kind of medical care during the previous } 12 \text { months (S4Q01 = Yes) OR who } \\
\text { needed some type of medical care during the past } 12 \text { months even though they did not see any health } \\
\text { care professional (S4Q02 = Yes). }\end{array}$} \\
\hline \multicolumn{2}{|c|}{ SAS Code and Annotation } \\
\hline \multicolumn{2}{|l|}{ Format } \\
\hline \multicolumn{2}{|c|}{ 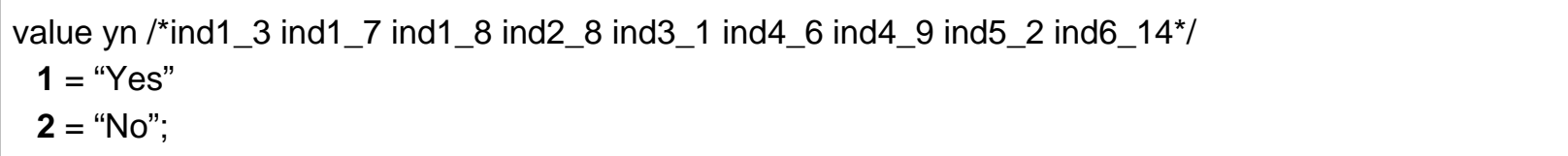 } \\
\hline \multicolumn{2}{|l|}{ Code } \\
\hline \multicolumn{2}{|c|}{$\begin{array}{l}\text { ind4_6 = .; } \\
\text { if s4Q07 eq } 0 \text { or s4Q02 eq } \mathbf{1} \text { then ind4_6 = } \mathbf{2} \text {; } \\
\text { if s4Q07 eq } \mathbf{1} \text { then ind4_6 = 1; } \\
\text { label ind4_6 = "Indicator 4.6: During the past } 12 \text { months, did (child's name) receive all the medical care } \\
\text { he/she needed? (S4Q07 - ages } 0-17 \text { who needed or used medical care)"; }\end{array}$} \\
\hline
\end{tabular}




\begin{tabular}{|c|c|}
\hline INDICATOR 4.7 & $\begin{array}{l}\text { During the past } 12 \text { months, did (child's name) receive all the } \\
\text { prescription medication }(\mathrm{Rx}) \text { helshe needed? }\end{array}$ \\
\hline Survey Items Used & S4Q17 \\
\hline Numerator & Children/youth who received all needed $\mathrm{Rx}$ \\
\hline Denominator & Children/youth ages 0-17 years who needed Rx during past 12 months \\
\hline Description & $\begin{array}{l}\text { Percent of children/youth ages } 0-17 \text { received all the Rx they needed during } \\
\text { the past } 12 \text { months (S4Q17 = Yes). }\end{array}$ \\
\hline \multicolumn{2}{|l|}{ Notes for Data-Users } \\
\hline \multicolumn{2}{|c|}{$\begin{array}{l}\text { Children/youth who "need prescription medication" is defined as children/youth who currently use or } \\
\text { have used Rx in the past } 12 \text { months }(\mathrm{S} 2 \mathrm{Q} 04 \text { or S4Q15 = Yes) OR children/youth who did not use any } \\
\mathrm{Rx} \text { in the past } 12 \text { months (S4Q16 = Yes), even though they needed it during that time (S4Q17 = No). } \\
\text { The number of children/youth reported to have needed Rx during the past } 12 \text { months, even though they } \\
\text { did not use any was } 1,785(\mathrm{~S} 4 \mathrm{Q} 16=\text { Yes). Of this group, } 154 \text { were reported as not having all of the Rx } \\
\text { they needed (S4Q17 = No). }\end{array}$} \\
\hline \multicolumn{2}{|c|}{ SAS Code and Annotation } \\
\hline \multicolumn{2}{|l|}{ Format } \\
\hline \multicolumn{2}{|c|}{$\begin{array}{l}\text { value prescrip } / * \text { ind4__7*/ } \\
\mathbf{1}=\text { ="Did not get all needed medication" } \\
\mathbf{2}=\text { "Got all needed medication"; }\end{array}$} \\
\hline \multicolumn{2}{|l|}{ Code } \\
\hline \multicolumn{2}{|c|}{$\begin{array}{l}\text { ind } 4 \_7=. ; \\
\text { if s4q17 eq } 0 \text { then ind4_7 = } 1 \\
\text { if s4q17 eq } 1 \text { then ind4_7 = } 2 \\
\text { if s4q17 gt } 1 \text { then ind4_7 = .; } \\
\text { label ind4_7 = "Indicator 4.7: During the past } 12 \text { months, did (child's name) receive all the Rx he/she } \\
\text { needed? (S4Q17 - ages } 0-17 \text { who needed Rx)"; }\end{array}$} \\
\hline
\end{tabular}




\begin{tabular}{|c|c|}
\hline INDICATOR 4.8 & $\begin{array}{l}\text { How many children/youth receive health care that meets the American } \\
\text { Academy of Pediatrics (AAP) definition of medical home? }\end{array}$ \\
\hline Survey Items Used & S4Q01 and S4Q03; SA5Q01 - S5Q13a \\
\hline Numerator & Children/youth whose health care meets AAP medical home criteria \\
\hline Denominator & Children/youth ages 0-17 years \\
\hline Description & $\begin{array}{l}\text { Percent of children/youth ages } 0-17 \text { who have a primary care provider (e.g., } \\
\text { personal doctor or nurse) AND one or more preventive care visits during past } \\
12 \text { months AND consistently receive all needed care from primary care } \\
\text { provider. }\end{array}$ \\
\hline Notes for Data-User & \\
\hline
\end{tabular}

The medical home composite measure and its sub-components are designed to operationalize the American Academy of Pediatrics (AAP) definition of "medical home." According to this definition, all children ideally should have access to "accessible, continuous, comprehensive, family centered, coordinated, compassionate, and culturally effective care within a medical home."

For additional information about the questions in the medical home section (Section 5) of the 2003 National Survey of Children's Health, go to:

www.cdc.gov/nchs/data/slaits/NSCH_Methodology_Report.pdf

We are indebted to Deborah Rosenberg, PhD, Research Associate Professor of Epidemiology, School of Public Health, University of Illinois at Chicago, for translating the SPSS Medical Home scoring program developed by CAHMI into SAS format.

\section{SAS Code and Annotation}

\section{Format}

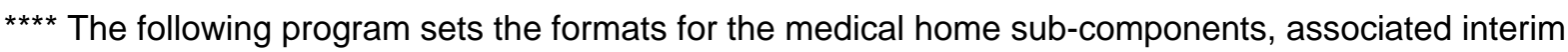
variables, and the overall medical home composite measure created by the SAS scoring program;

proc format library = library name;

value s5q0one /*format for PDN sub-component - S5Q01*/

$\mathbf{0}=$ 'No'

$1=$ 'Yes'

$6=$ 'DK'

$7=$ 'Refused';

value pc_tw /*format for: PREVENTIVE CARE VISIT sub-component - pc_2*/

$\mathbf{0}=$ 'Unknown'

$\mathbf{1}=$ 'No preventive care visit'

2 = 'One or more prev care visits';

value subcomp /*format for HOW OFTEN sub-components*/

$\mathbf{0}=$ 'Less than usually/always'

1 = 'Usually/always';

value acc_tw /*format for ACC_2 sub-component*/

$\mathbf{0}=$ 'Problems'

$\mathbf{1}=$ 'No problems'; 
value mh_yn /*format for MEDICAL HOME composite measure WITHOUT prev care visit added*/ $\mathbf{0}=$ 'No PDN or has PDN \& <75pts on 1 or more needed sub-comps'

1 = 'Has PDN \& 75pts or above on every needed sub-comp';

value ind4_8/*format for MEDICAL HOME composite measure*/

$\mathbf{1}=$ 'Care MEETS MEDICAL HOME definition'

2 = 'Care DOES NOT meet MEDICAL HOME definition';

run;

\section{Scoring program}

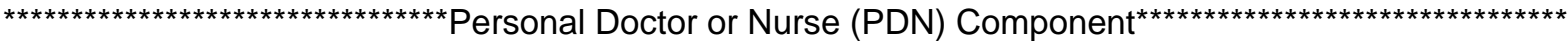

/**PERSONAL DOCTOR or NURSE sub-component of MEDICAL HOME composite measure ${ }^{\star \star / ~}$

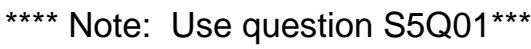

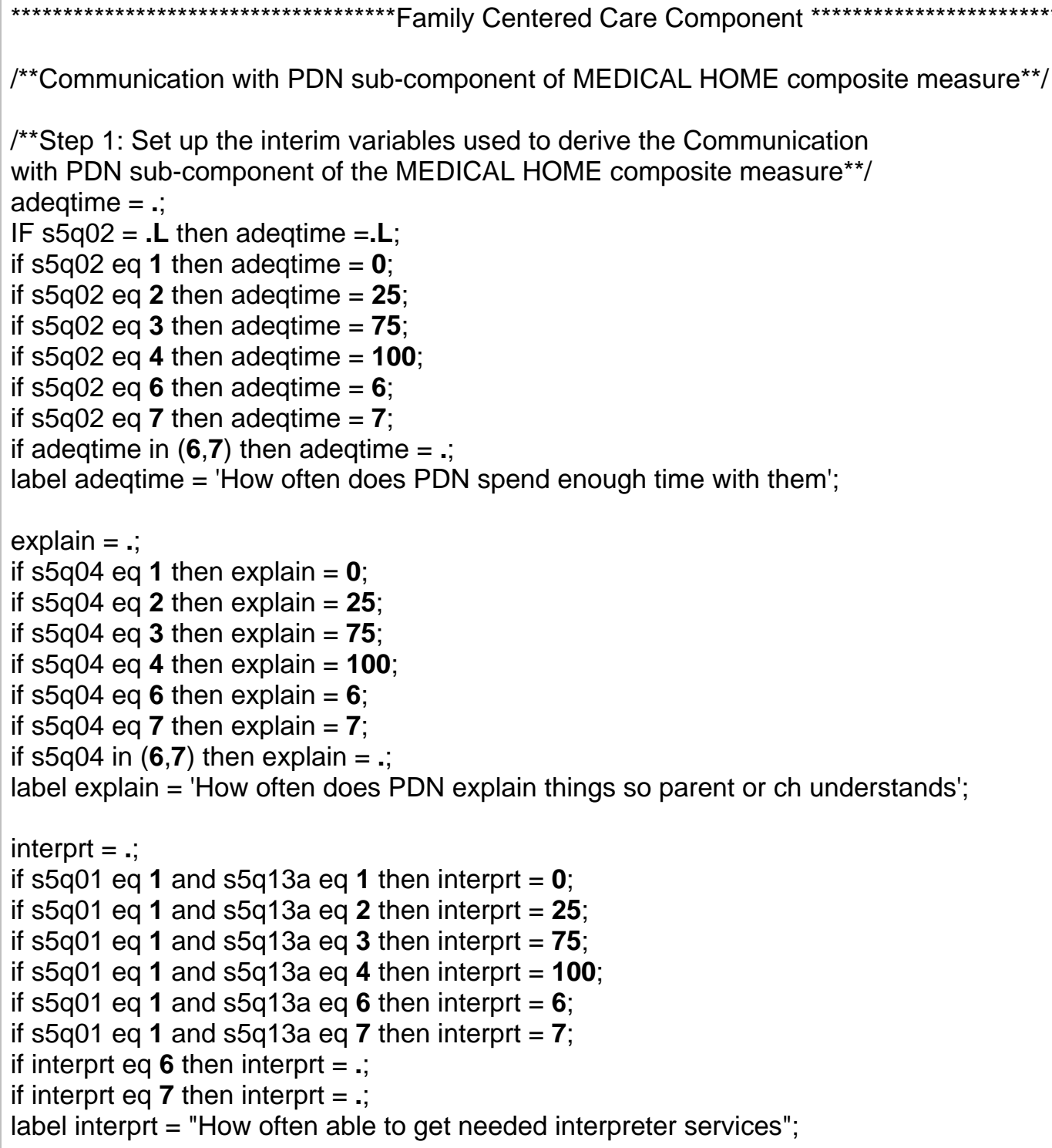


/**Step 2: Construct the "Communication with PDN" sub-component of MEDICAL HOME composite measure ${ }^{* \star} /$

pdn_cnt $=\mathbf{0}$;

if adeqtime in $(0,25,75,100)$ then pdn_cnt $=$ pdn_cnt $+\mathbf{1}$;

if explain in $(0,25,75,100)$ then pdn_cnt $=$ pdn_cnt $+\mathbf{1}$;

if interprt in $(\mathbf{0}, 25,75,100)$ then pdn_cnt $=$ pdn_cnt $+\mathbf{1}$;

label pdn_cnt = "Number of PDN communication elements needed ";

pdn_sum = sum(adeqtime, explain,interprt);

label pdn_sum = "Total points across all needed elements of comm w/ PDN";

if pdn_cnt ne 0 then do; $p d n 100=$ pdn_sum / pdn_cnt; end;

if pdn_cnt eq 0 then do; pdn100 = .; end;

label pdn100 = "Average score for communication with PDN sub-component of MEDICAL HOME";

pdncom_2 = ;

if pdn $100>=0$ and pdn100 $<=67$ then pdncom_2 $=0$;

if pdn100 > = 75 then pdncom_2 = 1;

label pdncom_2 = "PDN communication sub-component for MEDICAL HOME composite measure";

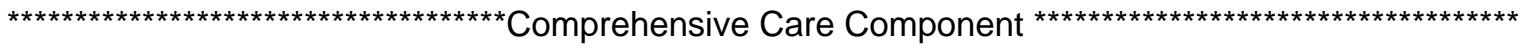

I**PREVENTIVE CARE VISIT sub-component of MEDICAL HOME composite measure**/

pc_2 =0.;

if s4q01 eq 0 or s4q03r eq 0 then pc_2 = 1;

if $s 4 q 03 r>=1$ then pc_2 = 2;

if s4q03rin $(96,97)$ then pc_2 $=0$;

label pc_2 = 'Preventive care visit sub-component';

/**GETTING NEEDED CARE/ADVICE sub-component of MEDICAL HOME composite measure**/

$1 * \star$ Step 1: The following syntax sets up the interim variables used to derive the "Getting needed care from PDN" sub-component of MEDICAL HOME composite measure ${ }^{\star \star /}$

phonehlp = :;

if s5q06a eq 1 then phonehlp $=\mathbf{0}$;

if $s 5 q 06 a$ eq 2 then phonehlp $=\mathbf{2 5}$;

if $s 5 q 06 a$ eq 3 then phonehlp $=\mathbf{7 5}$;

if s5q06a eq 4 then phonehlp $=\mathbf{1 0 0}$;

if s5q06a eq $\mathbf{6}$ then phonehlp $=\mathbf{6}$;

if s5q06a eq 7 then phonehlp = 7;

if s5q06a in $(6,7)$ then phonehlp = .;

label phonehlp = "How often get advice over phone from ch's PDN when needed";

getcare $=$;

if $\mathrm{s} 5 \mathrm{q} 07 \mathrm{a}$ eq $\mathbf{1}$ then getcare $=\mathbf{0}$;

if s5q07a eq 2 then getcare $=\mathbf{2 5}$;

if $55 q 07 a$ eq 3 then getcare $=\mathbf{7 5}$;

if $55 q 07$ a eq 4 then getcare $=100$;

if s5q07a eq $\mathbf{6}$ then getcare $=\mathbf{6}$;

if $s 5 q 07 a$ eq 7 then getcare $=7$;

if s5q07a in $(6,7)$ then getcare = ;

label getcare = "How often get needed care right away from ch's PDN"; 
/**Step 2: Construct the "GETTING NEEDED CARE/ADVICE" sub-component of MEDICAL HOME composite measure ${ }^{\star \star /}$

care_cnt $=\mathbf{0}$;

if phonehlp in $(0,25,75,100)$ then care_cnt $=$ care_cnt +1 ;

if getcare in $(0,25,75,100)$ then care $c n t=$ care $c n t+1$;

label care_cnt $=$ "Number of getting care elements needed";

care_sum $=\operatorname{sum}($ phonehlp,getcare $)$;

label care_sum = "Total points across needed elements of getting phone or urgent care from PDN";

if care_cnt ne 0 then do; care $100=$ care_sum / care_cnt; end;

if care ${ }^{-}$cnt eq 0 then do; care100 = .; end;

label care100 = "Average score for Getting Needed Care sub-component of MEDICAL HOME";

care_2 = ;

if care $100>=0$ and care $100<=67$ then care_2 $=0$;

if care100 $>=75$ then care_2 $=\mathbf{1}$;

label care_2 = "Getting Needed Care sub-component for MEDICAL HOME composite measure";

${ }^{* * A C C E S S}$ to SPECIALIST CARE \& SERVICES sub-component of MEDICAL HOME composite measure $^{\star \star 1}$

I**Step 1: The following syntax sets up the interim variables used to derive the "ACCESS to SPECIALIST CARE \& SERVICES" sub-component of MEDICAL HOME composite measure ${ }^{\star \star /}$ special = ;

if $s 5 q 09 a$ eq 1 then special $=\mathbf{0}$;

if s5q09a eq 2 then special $=\mathbf{2 5}$;

if s5q09a eq 3 then special $=\mathbf{7 5}$;

if s5q09a eq 4 then special $=\mathbf{1 0 0}$;

if s5q09a eq $\mathbf{6}$ then special $=\mathbf{6}$;

if $55 q 09$ a eq 7 then special $=7$;

if s5q09a in $(6,7)$ then special = .;

label special = "Problems getting needed care from specialist dr.";

services $=$;;

if $\mathrm{s} 5 \mathrm{q} 10 \mathrm{a}$ eq $\mathbf{1}$ then services $=\mathbf{0}$;

if $s 5 q 10$ a eq 2 then services $=\mathbf{2 5}$;

if $\mathrm{s} 5 \mathrm{q} 10 \mathrm{a}$ eq 3 then services $=\mathbf{7 5}$;

if $s 5 q 10 a$ eq 4 then services $=100$;

if $s 5 q 10 a$ eq 6 then services $=\mathbf{6}$;

if $s 5 q 10$ a eq 7 then services $=7$;

if s5q10a in $(6,7)$ then services = .;

label services = "Problems getting needed services or equip";

I**Step 2: Construct the "ACCESS to SPECIALIST CARE \& SERVICES" sub-component of MEDICAL HOME composite measure ${ }^{\star *}$

acc_cnt $=\mathbf{0}$;

if special in $(\mathbf{0}, \mathbf{2 5}, \mathbf{7 5}, \mathbf{1 0 0})$ then acc_cnt $=$ acc_cnt $+\mathbf{1}$;

if services in $(\mathbf{0}, 25,75,100)$ then acc $c n t=a c c \_c n t+1$;

label acc_cnt = "Number of access to care elements needed ";

acc_sum = sum(special, services);

label acc_sum = "Total points across accessing needed specialist care/services elements of MEDICAL HOME"; 


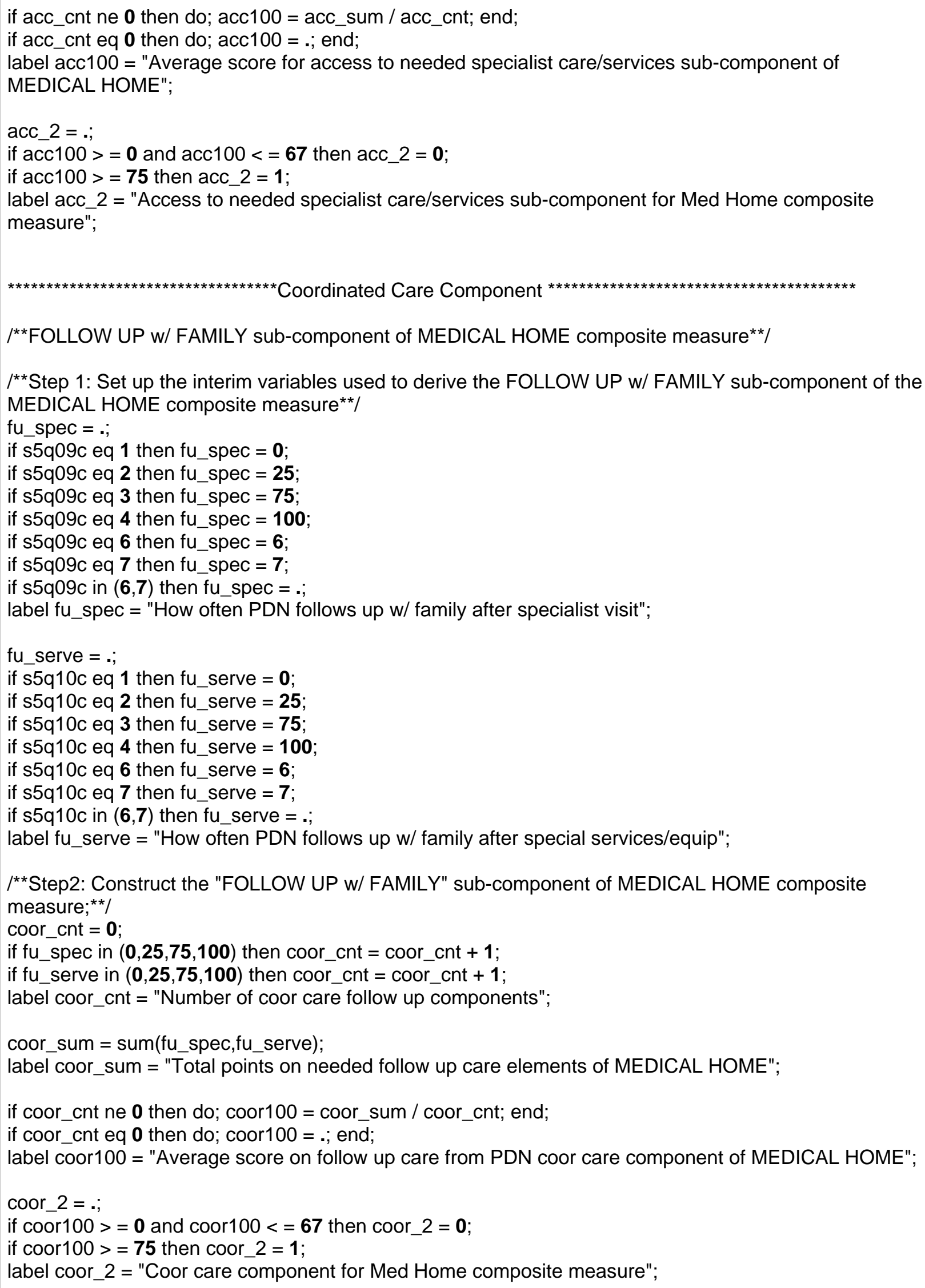




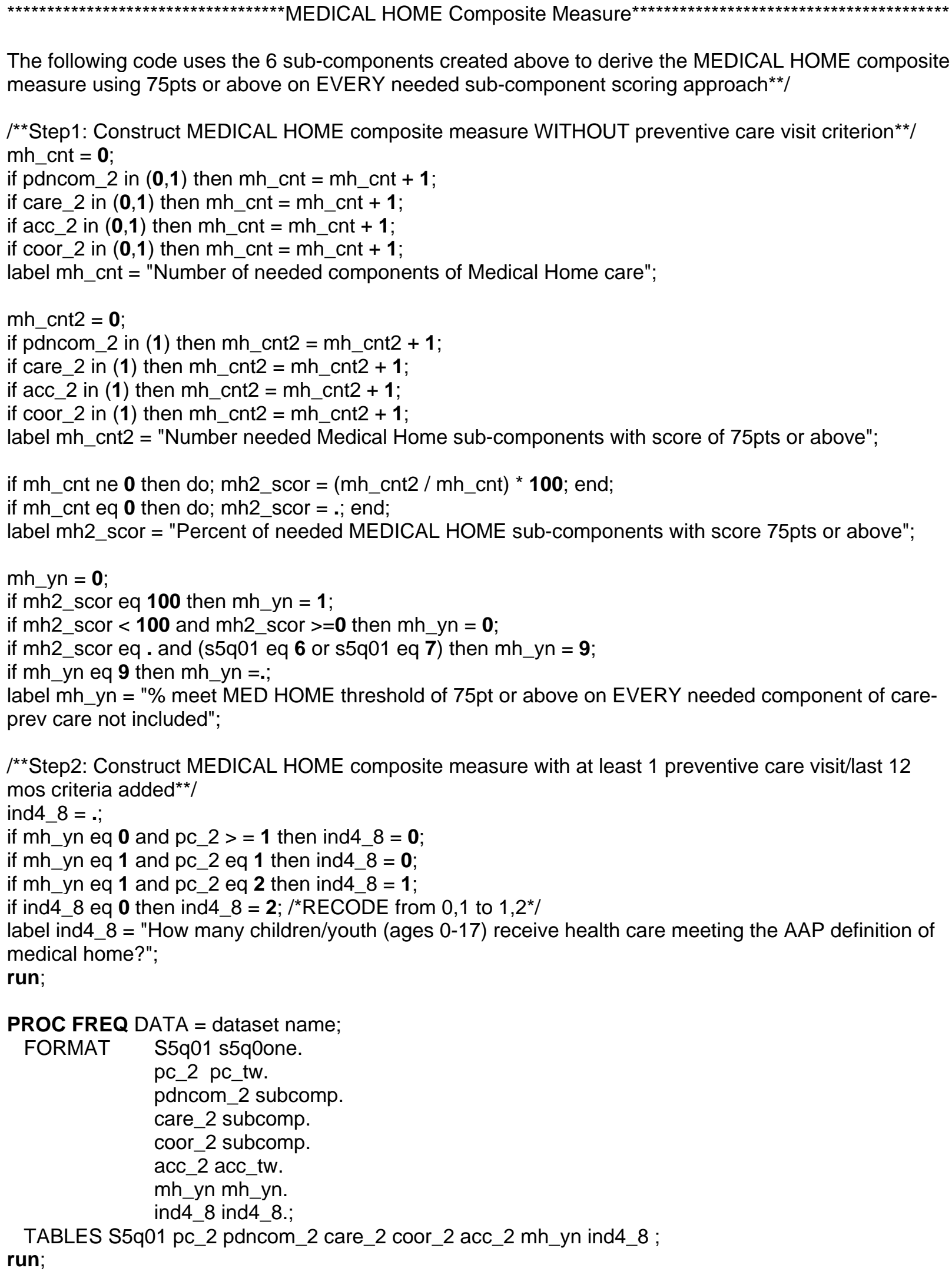

TABLES S5q01 pc_2 pdncom_2 care_2 coor_2 acc_2 mh_yn ind4_8 ; run; 


\begin{tabular}{|c|c|}
\hline INDICATOR 4.9 & $\begin{array}{l}\text { A personal doctor or nurse (PDN) is a health professional who knows } \\
\text { your child well and is familiar with your child's health history. Do you } \\
\text { have one or more persons you think of as (child's name)'s PDN? }\end{array}$ \\
\hline Survey Items Used & S5Q01 \\
\hline Numerator & Children/youth who have a PDN \\
\hline Denominator & Children/youth ages 0-17 years \\
\hline Description & $\begin{array}{l}\text { Percent of children/youth ages } 0-17 \text { with parents who have one or more } \\
\text { persons they think of as their child's PDN (S5Q01 = Yes). }\end{array}$ \\
\hline \multicolumn{2}{|l|}{ Notes for Data-Users } \\
\hline \multicolumn{2}{|c|}{$\begin{array}{l}\text { For more information about the scoring of this and other components of medical home derived from this } \\
\text { survey, email cahmi@ohsu.edu. }\end{array}$} \\
\hline \multicolumn{2}{|c|}{ SAS Code and Annotation } \\
\hline \multicolumn{2}{|l|}{ Format } \\
\hline \multicolumn{2}{|c|}{$\begin{array}{l}\text { value yn /*ind1_3 ind1_7 ind1_8 ind2_8 ind3_1 ind4_6 ind4_9 ind5_2 ind6_14*/ } \\
\begin{array}{l}1 \\
= \\
2\end{array}=\text { "Yes" }\end{array}$} \\
\hline \multicolumn{2}{|l|}{ Code } \\
\hline \multicolumn{2}{|c|}{$\begin{array}{l}\text { ind } 4 \_9=55 q 01 \text {; } \\
\text { if ind4_9 eq } 0 \text { then ind4_9=2; /*RECODE from } 0,1 \text { to } 1,2^{\star} / \\
\text { if ind4_9 in }(6,7) \text { then ind4_9=.; } \\
\text { label ind4_9 = "Indicator 4.9: A PDN is a health professional who knows your child well and is familiar } \\
\text { with your child's health history. Do you have one or more person(s) you think of as (child's) PDN? } \\
\text { (S5Q01 - ages 0-17)"; }\end{array}$} \\
\hline
\end{tabular}




\section{INDICATOR 4.10}

How many children/youth have a personal doctor or nurse (PDN) who

consistently spends enough time with them and explains things in ways that children and parents can understand?

Survey Items Used

Numerator

Denominator

Description
S5Q01; S5Q02 - S5Q04

Children/youth whose PDN consistently spends enough time and explains things

Children/youth ages 0-17 years

Percent of children/youth ages 0-17 whose PDN consistently spends enough time with them and explains things in a way that children and parents can understand. (S5Q01 = Yes; S5Q02 and S5Q04 = "Always" or "Usually").

\section{Notes for Data-Users}

Questions S5Q02 and S5Q04 are only asked for children who have one or more persons their parents consider to be the child's PDN (S5Q01 = Yes). PDN is a health professional who knows the child well and is familiar with the child's health history. This can be a general doctor, a pediatrician, a specialist doctor, a nurse practitioner, or a physician's assistant.

For more information about the scoring of the components of medical home derived from this survey, email cahmi@ohsu.edu.

\section{SAS Code and Annotation}

\section{Format}

value enotime $/ *$ ind4_10*/

$1=$ "Child does not have a personal dr/nurse"

$\mathbf{2}=$ "Personal $\mathrm{dr} /$ nurse does not consistently spend enough time or explain well"

3 = "Personal dr/nurse consistently spends enough time and explains well";

\section{Code}

I*IMPORTANT NOTE: Run the scoring program for Indicator 4.8 (Medical Home index) FIRST or the following code will not work because it uses the "Communication with PDN" scoring component of MEDICAL HOME Index to construct Indicator 4.10*/

if $s 5 q 01=0$ then ind4 $\_10=1$;

if pdncom_2 = 0 then ind4_10 = 2;

if pdncom_2 = 1 then ind4_10 = 3;

label ind4_10 = "Indicator 4.10: How many children/youth (ages 0-17) have a PDN who consistently spends enough time with them and explains things in ways that children and parents can understand?"; 


\begin{tabular}{|c|c|}
\hline INDICATOR 4.11 & $\begin{array}{l}\text { How many children/youth have a personal doctor or nurse (PDN) who is } \\
\text { consistently available when phone advice or urgent care is needed for } \\
\text { child? }\end{array}$ \\
\hline Survey Items Used & S5Q01; S5Q06 - S5Q07A \\
\hline Numerator & $\begin{array}{l}\text { Children/youth whose PDN is consistently available for phone advice and/or } \\
\text { urgent care }\end{array}$ \\
\hline Denominator & $\begin{array}{l}\text { Children/youth ages } 0-17 \text { years who needed phone advice and/or urgent } \\
\text { from their PDN during the past } 12 \text { months }\end{array}$ \\
\hline Description & $\begin{array}{l}\text { Among children/youth ages 0-17 with a PDN and who needed phone advice } \\
\text { and/or urgent care, percent whose PDN is consistently available when phone } \\
\text { advice or urgent care is needed (S5Q06A and/or S5Q07A = "Always" or } \\
\text { "Usually"). }\end{array}$ \\
\hline \multicolumn{2}{|l|}{ Notes for Data-Users } \\
\hline \multicolumn{2}{|c|}{$\begin{array}{l}\text { Only children who have a PDN }(\mathrm{S} 5 \mathrm{Q} 01=\text { Yes) and who needed phone advice and/or urgent care for an } \\
\text { injury or illness during the past } 12 \text { months (S5Q06 and S6Q07 = Yes) were asked the questions about } \\
\text { how often their PDN was available to give urgently needed care or phone advice. A PDN is a health } \\
\text { professional who knows the child well and is familiar with the child's health history. This can be a } \\
\text { general doctor, a pediatrician, a specialist doctor, a nurse practitioner, or a physician's assistant. } \\
\text { For more information about the scoring of the components of medical home derived from this survey, } \\
\text { email cahmi@ohsu.edu. }\end{array}$} \\
\hline \multicolumn{2}{|c|}{ SAS Code and Annotation } \\
\hline \multicolumn{2}{|l|}{ Format } \\
\hline \multicolumn{2}{|c|}{$\begin{array}{l}\text { value consist } / * \text { ind } 4 \_11^{*} / \\
\mathbf{1}=\text { "PDN consistently available" } \\
\mathbf{2}=\text { "PDN not consistently available"; }\end{array}$} \\
\hline \multicolumn{2}{|l|}{ Code } \\
\hline \multicolumn{2}{|c|}{ 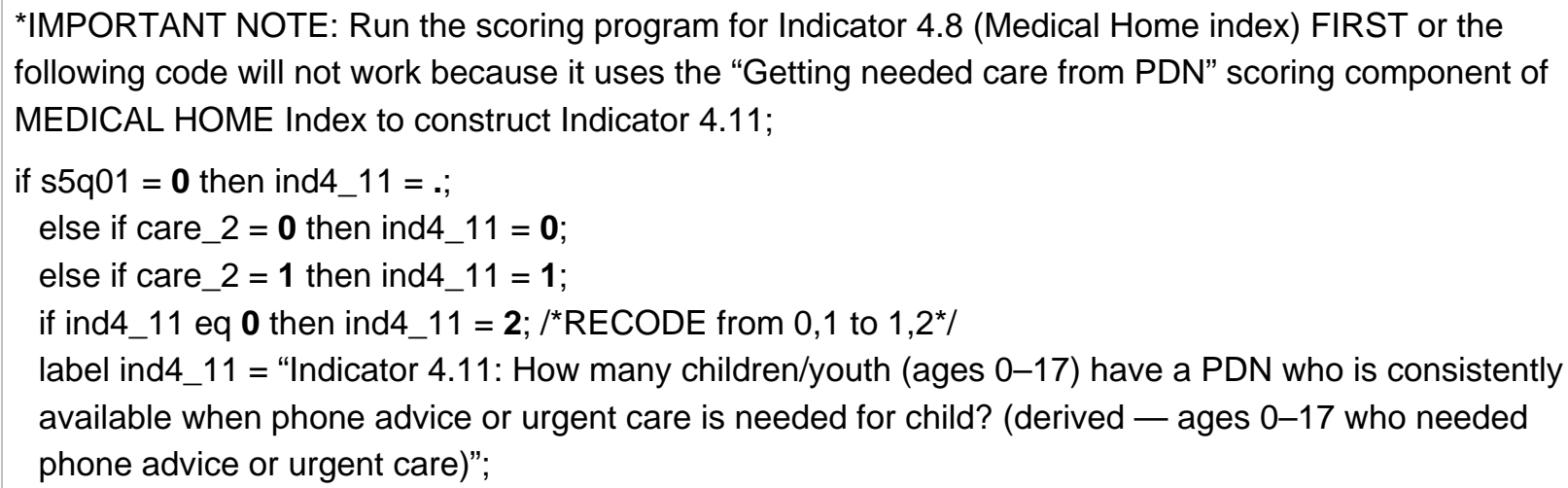 } \\
\hline
\end{tabular}




\begin{tabular}{|c|c|}
\hline INDICATOR 4.12 & $\begin{array}{l}\text { How many children/youth had problems getting the specialty care, } \\
\text { services, or equipment recommended by their personal doctor or nurse } \\
\text { (PDN)? }\end{array}$ \\
\hline Survey Items Used & S5Q01; S5Q09 - S5Q09A; and/or S5Q10 - S5Q10A \\
\hline Numerator & $\begin{array}{l}\text { Children/youth who experienced significant problems getting recommended } \\
\text { care }\end{array}$ \\
\hline Denominator & $\begin{array}{l}\text { Children/youth ages } 0-17 \text { years who needed specialist care and/or } \\
\text { specialized services or equipment recommended by their PDN during the } \\
\text { past } 12 \text { months }\end{array}$ \\
\hline Description & $\begin{array}{l}\text { Among children/youth ages } 0-17 \text { who have a PDN and needed specialist } \\
\text { care and/or specialized services or equipment recommended by their PDN, } \\
\text { percent who experienced problems getting them (S5Q09A and/or S5Q10A = } \\
\text { "a big problem" or "a moderate problem"). }\end{array}$ \\
\hline \multicolumn{2}{|l|}{ Notes for Data-Users } \\
\hline \multirow{2}{*}{\multicolumn{2}{|c|}{$\begin{array}{l}\text { Only children who have a PDN (S5Q01 = Yes) and who needed specialist care and/or specialized health } \\
\text { services or equipment during the past } 12 \text { months (S5Q09 and S5Q10 = Yes) were asked the questions } \\
\text { about level of problems experienced getting needed care. A PDN is a health professional who knows the } \\
\text { child well and is familiar with the child's health history. This can be a general doctor, a pediatrician, a } \\
\text { specialist doctor, a nurse practitioner, or a physician's assistant. } \\
\text { For more information about the scoring of the components of medical home derived from this survey, } \\
\text { email cahmi@ohsu.edu. }\end{array}$}} \\
\hline & \\
\hline \multicolumn{2}{|c|}{ SAS Code and Annotation } \\
\hline \multicolumn{2}{|l|}{ Format } \\
\hline \multicolumn{2}{|c|}{$\begin{array}{l}\text { value reccare } / * \text { ind } 4 \_12^{*} / \\
\begin{aligned} \mathbf{1} & =\text { "Few or no problems getting recommended care" } \\
\mathbf{2} & =\text { "Significant problems getting recommended care"; }\end{aligned}\end{array}$} \\
\hline \multicolumn{2}{|l|}{ Code } \\
\hline \multicolumn{2}{|c|}{ 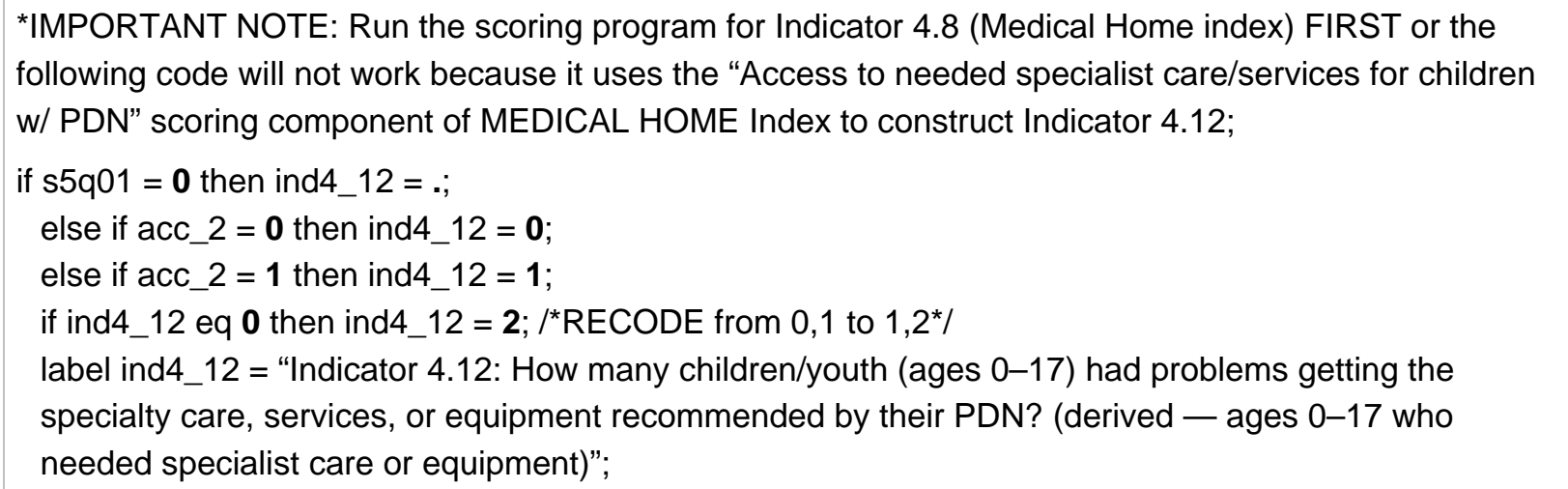 } \\
\hline
\end{tabular}




\begin{tabular}{|c|c|}
\hline INDICATOR 4.13 & $\begin{array}{l}\text { How many children/youth have a personal doctor or nurse (PDN) who } \\
\text { follows up with family after child sees specialist or gets specialized } \\
\text { services/equipment? }\end{array}$ \\
\hline Survey Items Used & S5Q01; S5Q09C and/or S5Q10C \\
\hline Numerator & $\begin{array}{l}\text { Children/youth whose PDN consistently follows up after specialized care or } \\
\text { equipment }\end{array}$ \\
\hline Denominator & $\begin{array}{l}\text { Children/youth ages 0-17 years who have a PDN and needed specialist care } \\
\text { or specialized services/equipment during the past } 12 \text { months }\end{array}$ \\
\hline Description & $\begin{array}{l}\text { Among children/youth ages 0-17 who have a PDN and got specialist care or } \\
\text { specialized services/equipment, percent whose PDN consistently follows up } \\
\text { with family (S5Q09C and/or S5Q10C = "Usually" or "Always"). }\end{array}$ \\
\hline \multicolumn{2}{|l|}{ Notes for Data-Users } \\
\hline \multirow{2}{*}{\multicolumn{2}{|c|}{$\begin{array}{l}\text { Only children who have a PDN (S5Q01 = Yes) and who needed specialist care and/or specialized health } \\
\text { services or equipment during the past } 12 \text { months (S5Q09 and S5Q10 = Yes) were asked the questions } \\
\text { about how often their PDN followed up with the family after child received care. A PDN is a health } \\
\text { professional who knows the child well and is familiar with the child's health history. This can be a } \\
\text { general doctor, a pediatrician, a specialist doctor, a nurse practitioner, or a physician's assistant. } \\
\text { For more information about the scoring of the components of medical home derived from this survey, } \\
\text { email cahmi@ohsu.edu. }\end{array}$}} \\
\hline & \\
\hline \multicolumn{2}{|c|}{ SAS Code and Annotation } \\
\hline \multicolumn{2}{|l|}{ Format } \\
\hline \multicolumn{2}{|c|}{$\begin{array}{l}\text { value followup } / * \text { ind } 4 \_13^{\star} / \\
\mathbf{1}=\text { "PDN consistently follows up" } \\
\mathbf{2}=\text { "PDN does not consistently follow up"; }\end{array}$} \\
\hline \multicolumn{2}{|l|}{ Code } \\
\hline \multicolumn{2}{|c|}{ 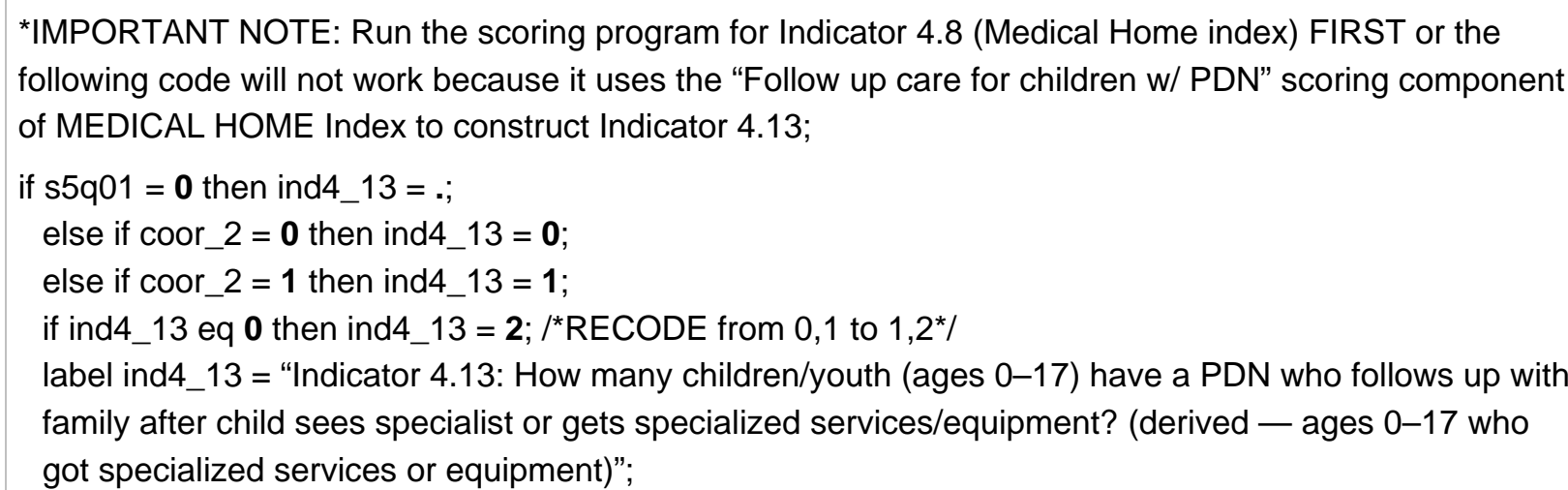 } \\
\hline
\end{tabular}




\begin{tabular}{|c|c|}
\hline INDICATOR 4.14 & $\begin{array}{l}\text { During the past } 12 \text { months, did (child's name)'s doctors or other health } \\
\text { care professionals ask if you have concerns about his/her learning, } \\
\text { development, or behavior? }\end{array}$ \\
\hline Survey Items Used & S6Q28 \\
\hline Numerator & $\begin{array}{l}\text { Young children who had health care visit at which a health care professional } \\
\text { asked parents about concerns regarding child's learning, development or } \\
\text { behavior }\end{array}$ \\
\hline Denominator & Children ages $0-5$ years \\
\hline Description & $\begin{array}{l}\text { Percent of children ages } 0-5 \text { whose doctors asked if parents have concerns } \\
\text { about child's learning, development, or behavior (S6Q28 = Yes). }\end{array}$ \\
\hline \multicolumn{2}{|l|}{ Notes for Data-Users } \\
\hline \multicolumn{2}{|c|}{ SAS Code and Annotation } \\
\hline \multicolumn{2}{|c|}{ Format } \\
\hline \multicolumn{2}{|c|}{$\begin{array}{l}\text { value dr_asks /*ind4_14*/ } \\
\mathbf{1}=\text { = "Visited dr, asked about concerns" } \\
\mathbf{2}=\text { "Visited dr, but not asked" } \\
\mathbf{3}=\text { ="Child did not visit doctor"; }\end{array}$} \\
\hline \multicolumn{2}{|l|}{ Code } \\
\hline \multicolumn{2}{|c|}{$\begin{array}{l}\text { ind4_14 = s6q28; } \\
\text { if ageyr_child }<\mathbf{6} \text { and s4q01 eq } \mathbf{0} \text { then ind4_14 = 3; } \\
\text { if ind4_14 in }(\mathbf{6}, 7) \text { then ind4_14 = .; } \\
\text { if ind4_14 eq } \mathbf{0} \text { then ind4_14 = 2; / RECODE from } 0,1 \text { to } 1,2^{\star} / \\
\text { label ind4_14 = "Indicator } 4.14 \text { : During the past } 12 \text { months, did (child's name)'s doctors or other health } \\
\text { care professionals ask if you have concerns about his/her learning, development, or behavior? (S6Q28 } \\
\text { — ages } 0-5) " ;\end{array}$} \\
\hline
\end{tabular}




\begin{abstract}
INDICATOR 4.15
During the past 12 months, did (child's name)'s doctors give you

specific information to address your concerns about his/her learning, development, or behavior?

\begin{tabular}{|l|l|}
\hline Survey Items Used & S6Q08 - S6Q27; S6Q29 \\
\hline Numerator & $\begin{array}{l}\text { Children whose parents were highly concerned and got needed information } \\
\text { from the child's doctor }\end{array}$ \\
\hline Denominator & $\begin{array}{l}\text { Children ages 0-5 years whose parents were highly concerned about their } \\
\text { child's learning, development, or behavior }\end{array}$ \\
\hline Description & $\begin{array}{l}\text { Percent of children ages 0-5 whose parents were highly concerned about } \\
\text { their learning, development or behavior and got information addressing their } \\
\text { concerns from child's doctor (S6Q29 = Yes). }\end{array}$ \\
\hline
\end{tabular}

Notes for Data-Users

Question S6Q29 was only asked for children whose parents answered "A lot concerned" to any of questions S6Q09 through S6Q27 and/or "Yes" to question S6Q08.

The PEDS (Parents' Evaluation of Developmental Status) is a standardized tool designed to identify children at risk for developmental, behavioral, or social delays. The NSCH uses the non-clinical version of the PEDS. Researchers interested in analyzing NSCH data collected with the PEDS instrument will need to consult PEDS documentation for scoring instructions at www.pedstest.com. Health care providers interested in using the PEDS to assess or make decisions about the developmental status for individual children, need to use the clinical version of the PEDS instrument, which can be obtained from Ellsworth \& Vandermeer Press, LLC. National data for the PEDS are also available from the 2000 National Survey of Early Childhood Health. For more information, see www.cdc.gov/nchs/data/slaits/NSCH_Methodology_Report.pdf.

\section{SAS Code and Annotation}

Format

value needinfo/*ind4_15*/

$1=$ "Highly concerned AND got needed info"

2 = "Highly concerned but did not get info";

\title{
Code
}

ind4_15 = s6q29;

if ind4_15 in $(6,7)$ then ind4_15 = .;

if ind4_15 eq 0 then ind4_15 = 2; /*RECODE from 0,1 to $1,2^{*} /$

label ind4_15 = "Indicator 4.15: During the past 12 months, did (child's name)'s doctors give you specific information to address your concerns about his/her learning, development, or behavior? (S6Q29 - ages 0-5 with highly concerned parents)"; 


\begin{tabular}{|c|c|}
\hline INDICATOR 5.1 & $\begin{array}{l}\text { How many children regularly attended nursery school, preschool, } \\
\text { kindergarten, Head Start, or Early Start during the past month? }\end{array}$ \\
\hline Survey Items Used & S6Q51 and S6Q52 \\
\hline Numerator & Children who regularly attended early childhood education \\
\hline Denominator & Children ages $3-5$ years \\
\hline Description & $\begin{array}{l}\text { Percent of children ages } 3-5 \text { who regularly attended nursery school, preschool, } \\
\text { kindergarten and/or Head Start or Early Start during the past month (S6Q51 } \\
\text { and/or S6Q52 = Yes). }\end{array}$ \\
\hline \multicolumn{2}{|l|}{ Notes for Data-Users } \\
\hline \multicolumn{2}{|c|}{$\begin{array}{l}\text { Question S2Q51 (nursery school, preschool, or kindergarten attendance) is only asked for children ages } \\
\text { 3-5 years. Question S2Q52 (Head Start or Early Start attendance) is asked for children ages } 0-5 \text { years; } \\
\text { however, responses for children ages 3-5 years only are included in the denominator for this indicator. }\end{array}$} \\
\hline \multicolumn{2}{|c|}{ SAS Code and Annotation } \\
\hline \multicolumn{2}{|l|}{ Format } \\
\hline \multicolumn{2}{|c|}{$\begin{array}{l}\text { value early_sc /*ind5_1*/ } \\
\mathbf{1}=\text { = "Regularly attended during past month" } \\
\mathbf{2}=\text { ="Did not attend during past month" } \\
\mathbf{9 9}=\text { = "DK or Refused both items"; }\end{array}$} \\
\hline \multicolumn{2}{|l|}{ Code } \\
\hline \multicolumn{2}{|c|}{$\begin{array}{l}\text { ind5_1 = ; } \\
\text { if S6Q52 eq } 0 \text { and S6Q51 eq } 0 \text { then ind5_1 = 2; /*Did not attend*I } \\
\text { if (S6Q51 eq } 1 \text { or S6Q52 eq } 1 \text { ) and (AGEYR_CHILD> = 3) then ind5_1 = 1; /*Regularly attended*/ } \\
\text { if (S6Q51 eq } 6 \text { or s6q51 eq 7) and (s6q52 eq } 6 \text { or s6q52 eq 7) then ind5_1 = .; } \\
\text { label ind5_1 = "Indicator 5.1: How many children (ages 3-5) regularly attended nursery school, } \\
\text { preschool, kindergarten, Head Start, or Early Start during the past month? (derived)"; }\end{array}$} \\
\hline
\end{tabular}




\begin{tabular}{|c|c|}
\hline INDICATOR 5.2 & Since starting kindergarten, has (child's name) repeated any grades? \\
\hline Survey Items Used & S7Q09 \\
\hline Numerator & $\begin{array}{l}\text { Children/youth who have repeated one or more grades since starting } \\
\text { kindergarten }\end{array}$ \\
\hline Denominator & Children/youth ages $6-17$ years \\
\hline Description & $\begin{array}{l}\text { Percent of children/youth ages } 6-17 \text { who repeated one or more grades since } \\
\text { starting kindergarten (S7Q09 = Yes). }\end{array}$ \\
\hline \multicolumn{2}{|l|}{ Notes for Data-Users } \\
\hline \multicolumn{2}{|c|}{$\begin{array}{l}\text { Question S7Q09 was asked for all children/youth currently enrolled in public or private schools, for those } \\
\text { who were not enrolled in any school at the time of the survey (S7Q01 = Yes OR S7Q01F = Yes), and for } \\
\text { children were home-schooled during the past year. }\end{array}$} \\
\hline \multicolumn{2}{|c|}{ SAS Code and Annotation } \\
\hline \multicolumn{2}{|l|}{ Format } \\
\hline \multicolumn{2}{|c|}{$\begin{array}{l}\text { value yn /*ind1_3 ind1_7 ind1_8 ind2_8 ind3_1 ind4_6 ind4_9 ind5_2 ind6_14*/ } \\
\mathbf{1}=\text { =Yes" } \\
\mathbf{2}=\text { ="No"; }\end{array}$} \\
\hline \multicolumn{2}{|l|}{ Code } \\
\hline \multicolumn{2}{|c|}{$\begin{array}{l}\text { ind5_2 = ; } \\
\text { if s7q09 eq } 1 \text { then ind5_2 = 1; /*Yes*/ } \\
\text { if s7q09 eq } 0 \text { then ind5_2 = 2; /*No*/ } \\
\text { if s7q09 in }(6,7) \text { then ind5_2 }=. ; / /^{*} \text { Don't Know and Refused set to missing*/ } \\
\text { label ind5_2 = "Indicator 5.2: Since starting kindergarten, has (child's name) repeated any grades? } \\
\text { (S7Q09 - ages 6-17)"; }\end{array}$} \\
\hline
\end{tabular}




\begin{tabular}{|c|c|}
\hline INDICATOR 5.3 & $\begin{array}{l}\text { Did (child's name) participate in one or more organized activities outside } \\
\text { of school, such as sports teams or lessons, clubs, or religious groups? }\end{array}$ \\
\hline Survey Items Used & S7Q10, S7Q11 or S7Q11a \\
\hline Numerator & $\begin{array}{l}\text { Children/youth who participated in one or more organized activities outside of } \\
\text { school }\end{array}$ \\
\hline Denominator & Children/youth ages 6-17 years \\
\hline Description & $\begin{array}{l}\text { Percent of children/youth ages } 6-17 \text { who participated in one or more organized } \\
\text { activities outside of school (S7Q10 = Yes or S7Q11 = Yes or (S7Q11a = Yes). }\end{array}$ \\
\hline \multicolumn{2}{|l|}{ Notes for Data-Users } \\
\hline \multicolumn{2}{|c|}{$\begin{array}{l}\text { Activities outside of school are defined as: sports teams or lessons } \\
\text { Scouts, religious groups or any other organized event or activity the } \\
\text { weekends. }\end{array}$} \\
\hline \multicolumn{2}{|c|}{ SAS Code and Annotation } \\
\hline \multicolumn{2}{|l|}{ Format } \\
\hline \multicolumn{2}{|c|}{$\begin{array}{l}\text { value out_sch /*ind5_3*/ } \\
\mathbf{1}=\text { ="Participated in } 1 \text { or more organized activities" } \\
\mathbf{2}=\text { "Did not participate in organized activities" } \\
\mathbf{9 9}=\text { ="DK or Refused on all three items"; }\end{array}$} \\
\hline \multicolumn{2}{|l|}{ Code } \\
\hline \multicolumn{2}{|c|}{ 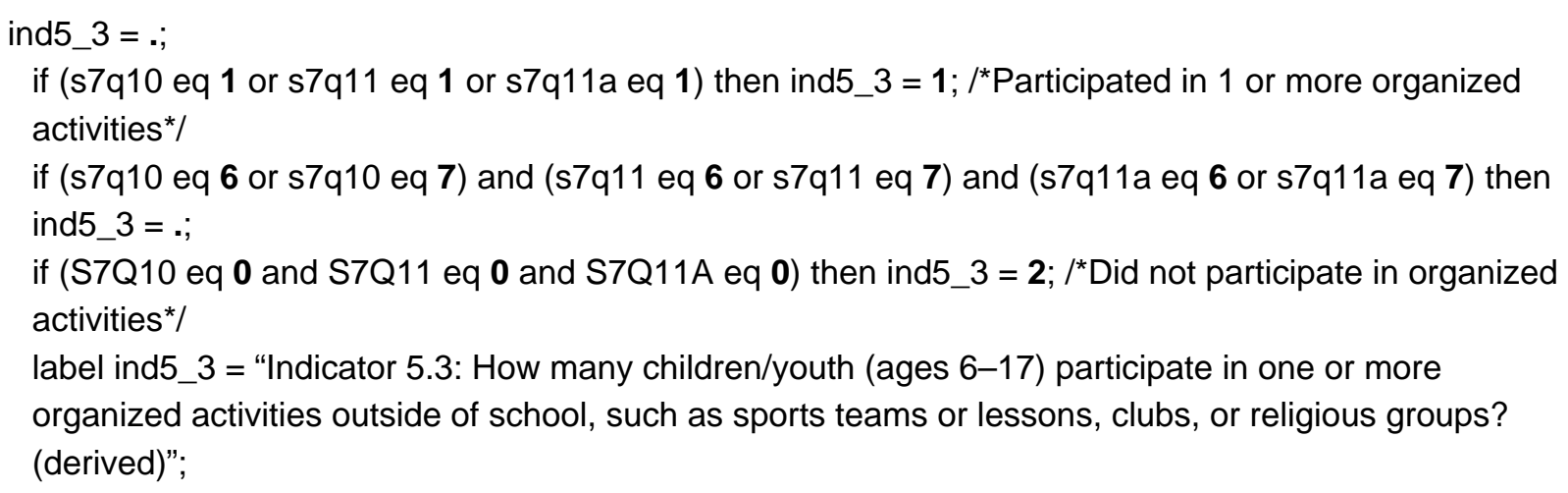 } \\
\hline
\end{tabular}




\begin{tabular}{|c|c|}
\hline INDICATOR 5.4 & $\begin{array}{l}\text { During the past } 12 \text { months, has (child's name) been involved in any type } \\
\text { of community service or volunteer work at school, church, or in the } \\
\text { community? }\end{array}$ \\
\hline Survey Items Used & S7Q17 \\
\hline Numerator & $\begin{array}{l}\text { Children/youth who participated in any type of community service or volunteer } \\
\text { work }\end{array}$ \\
\hline Denominator & Children/youth ages $12-17$ years \\
\hline Description & $\begin{array}{l}\text { Percent of children/youth ages } 12-17 \text { who participated in any type of } \\
\text { community service or volunteer work during the past } 12 \text { months (S7Q17 = Yes). }\end{array}$ \\
\hline \multicolumn{2}{|l|}{ Notes for Data-Users } \\
\hline \multicolumn{2}{|c|}{ SAS Code and Annotation } \\
\hline \multicolumn{2}{|l|}{ Format } \\
\hline \multicolumn{2}{|c|}{$\begin{array}{l}\text { value vol_act } / * \text { ind5_4*/ } \\
\mathbf{1}=\text { ="Participated in volunteer work" } \\
\mathbf{2}=\text { "Did NOT participate"; }\end{array}$} \\
\hline \multicolumn{2}{|l|}{ Code } \\
\hline \multicolumn{2}{|c|}{$\begin{array}{l}\text { ind5_4 = S7Q17; } \\
\text { if S7Q17 eq } 0 \text { then ind5_4 = 2; /*Did NOT participate*/ } \\
\text { if S7Q17 eq } 1 \text { then ind5_4 =1; /*Participated in volunteer work*/ } \\
\text { if S7Q17 in }(6,7) \text { then ind5_4 = .; } \\
\text { label ind5_4 = "Indicator 5.4: During the past } 12 \text { months, has (child's name) been involved in any type } \\
\text { of community service or volunteer work at school, church, or in the community? (S7Q17 - } \\
\text { ages 12-17)"; }\end{array}$} \\
\hline
\end{tabular}




\begin{tabular}{|c|c|}
\hline INDICATOR 5.5 & During the past week, how many hours did (child's name) work for pay? \\
\hline Survey Items Used & S7Q19 \\
\hline Numerators & $\begin{array}{l}\text { - Youth did not work for pay during the past week } \\
\text { - } \quad \text { Youth who worked for pay } 1-9 \text { hours during the past week } \\
\text { - } \quad \text { Youth who worked for pay } 10 \text { or more hours during the past week }\end{array}$ \\
\hline Denominator & Youth ages $12-17$ years \\
\hline Description & $\begin{array}{l}\text { Percent of youth ages } 12-17 \text { who did not work for pay, or who worked for pay } \\
1-9 \text { hours, or } 10 \text { or more hours. }\end{array}$ \\
\hline \multicolumn{2}{|l|}{ Notes for Data-Users } \\
\hline \multicolumn{2}{|c|}{ "Working for pay" only includes work done outside the home. } \\
\hline \multicolumn{2}{|c|}{ SAS Code and Annotation } \\
\hline \multicolumn{2}{|l|}{ Format } \\
\hline \multicolumn{2}{|c|}{$\begin{array}{l}\text { value workpay } / * \text { ind5_5*/ } \\
\begin{aligned} \mathbf{1} & =\text { "Did not work" } \\
\mathbf{2} & =\text { "Less than } 1 \text { hour-9 hours" } \\
\mathbf{3} & =\text { "10 hours or more"; }\end{aligned}\end{array}$} \\
\hline \multicolumn{2}{|l|}{ Code } \\
\hline \multicolumn{2}{|c|}{ 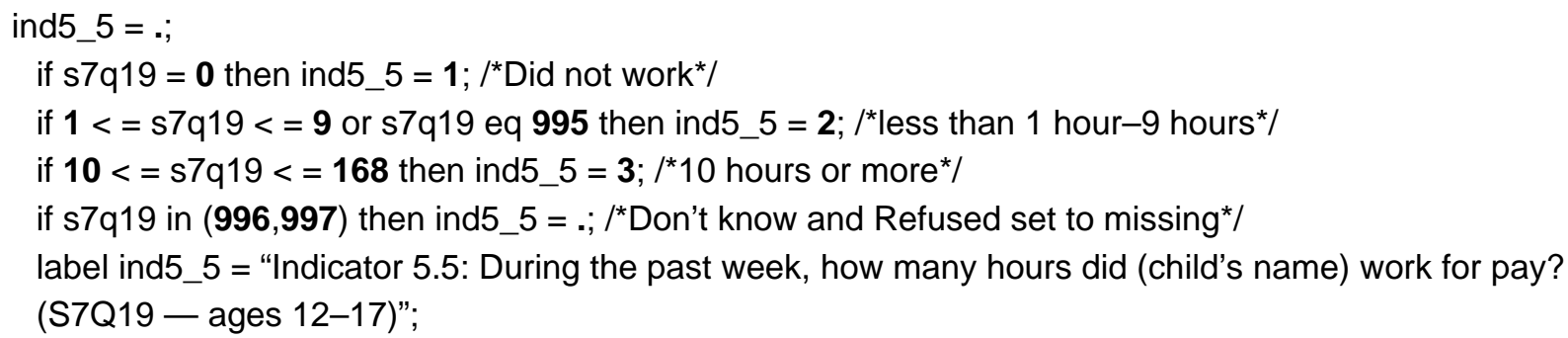 } \\
\hline
\end{tabular}




\begin{tabular}{|l|l|}
\hline INDICATOR 5.6 & $\begin{array}{l}\text { On an average school day, about how much time does (child's name) } \\
\text { usually spend reading for pleasure? }\end{array}$ \\
\hline Survey Items Used & S7Q26 \\
\hline Numerators & $\begin{array}{l}\text { Children/youth who do not read on an average school day } \\
\text { - }\end{array}$ \\
\hline $\begin{array}{l}\text { - Children/youth who read less than } 30 \text { minutes on an average school day } \\
\text { - Children/youth who read 30-60 minutes on an average school day }\end{array}$ \\
\hline Denominator & Children/youth ages 6-17 years \\
\hline Description & $\begin{array}{l}\text { Percent of children/youth ages 6-17 who do not read for pleasure, or who read } \\
\text { for less than } 30 \text { minutes, 30-60 minutes, or more than } 60 \text { minutes on an } \\
\text { average school day. }\end{array}$ \\
\hline
\end{tabular}

Notes for Data-Users

"Reading for pleasure" is defined as time when child reads to him/herself or is read to by another person. It does not include listening to books read by or with the help of an audiotape, record, $C D$, or computer.

\section{SAS Code and Annotation}

\section{Format}

value read_ple/*ind5_6*/

$1=$ "None"

$2=$ "30 minutes or less"

$3=$ "31-60 minutes"

4 = "Over 60 minutes";

\section{Code}

ind5_6 = .;

S7Q26r2 = S7Q26;

if S7Q26 eq 995 then $\mathrm{S} 7 \mathrm{Q} 26 \mathrm{r} 2 \mathbf{= 0 . 5}$;

if S7Q26r2 in $(996,997)$ then S7Q26r2 = .;

S7Q26r3 = S7Q26r2;

if S7Q26r3 in $(\mathbf{9 9 6}, \mathbf{9 9 7})$ then S7Q26r3 = .;

if S7Q26a eq 1 then do S7Q26r3 = S7Q26r2*60; end;

if S7Q26r3 eq 0 then ind5_6 = 1; /*None*/

if S7Q26r3 ge $\mathbf{0 . 5}$ and S7Q26r3 le $\mathbf{3 0}$ then ind5_6 = 2; /*30 minutes or less*/

if S7Q26r3 ge 31 and S7Q26r3 le 60 then ind5_6 = 3; /*31-60 minutes*/

if S7Q26r3 ge 61 and S7Q26r3 le 1800 then ind5_6 = 4; /*Over 60 minutes*/

label ind5_6 = "Indicator 5.6: On an average school day, about how much time does (child's name) usually spend reading for pleasure? (S7Q26 - ages 6-17)"; 


\begin{tabular}{|c|c|}
\hline INDICATOR 5.7 & How often does (child's name) attend religious services? \\
\hline Survey Items Used & S8Q02 and S8Q02a \\
\hline Numerators & $\begin{array}{l}\text { - } \quad \text { Children/youth who do not attend religious services } \\
\text { - } \quad \text { Children/youth who attend religious services once a year } \\
\text { - } \quad \text { Children/youth who attend religious services once a month } \\
\text { - Children/youth who attend religious services once a week or more often }\end{array}$ \\
\hline Denominator & Children/youth ages $0-17$ years \\
\hline Description & $\begin{array}{l}\text { Percent of children/youth ages } 0-17 \text { who do not attend religious services, or } \\
\text { who attend religious services at least once per year (but less than once per } \\
\text { month), at least once per month (but less than once per week), or once per } \\
\text { week or more. }\end{array}$ \\
\hline \multicolumn{2}{|l|}{ Notes for Data-Users } \\
\hline \multicolumn{2}{|c|}{ SAS Code and Annotation } \\
\hline \multicolumn{2}{|l|}{ Format } \\
\hline \multicolumn{2}{|c|}{ 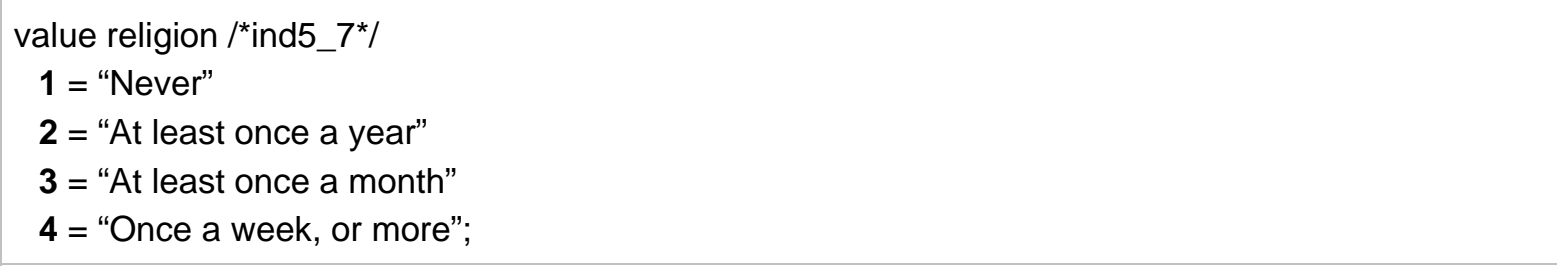 } \\
\hline \multicolumn{2}{|l|}{ Code } \\
\hline \multicolumn{2}{|c|}{ 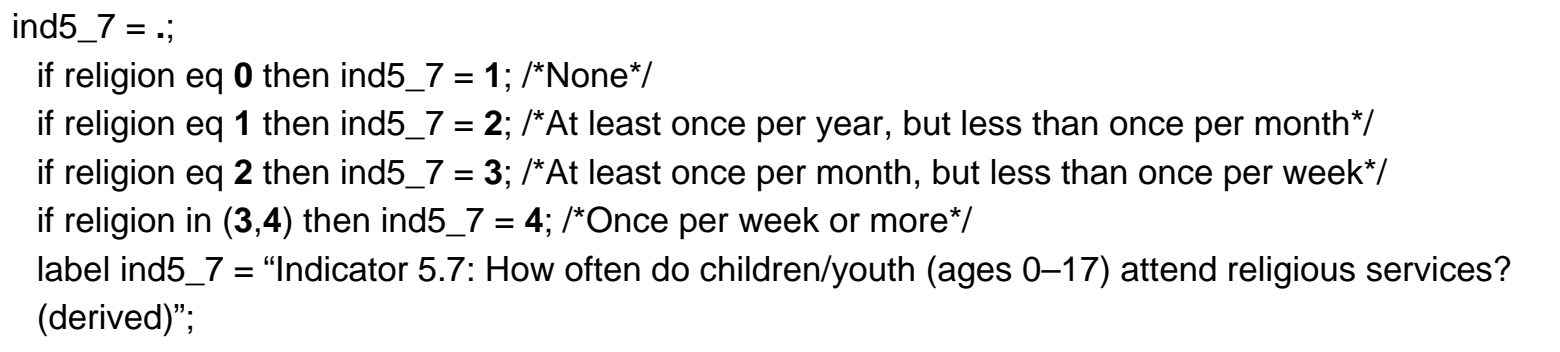 } \\
\hline
\end{tabular}




\begin{tabular}{|c|c|}
\hline INDICATOR 6.1 & $\begin{array}{l}\text { Would you say that in general (child's mother's name)'s health is } \\
\text { excellent, very good, good, fair, or poor? }\end{array}$ \\
\hline Survey Items Used & S9Q08 \\
\hline Numerators & $\begin{array}{l}\text { - Children/youth whose mother's general health is excellent or very good } \\
\text { - Children/youth whose mother's general health is good } \\
\text { - Children/youth whose mother's general health is fair or poor }\end{array}$ \\
\hline Denominator & $\begin{array}{l}\text { Children/youth ages 0-17 years who live with their biological, step, foster or } \\
\text { adoptive mothers }\end{array}$ \\
\hline Description & $\begin{array}{l}\text { Percent of children ages 0-17 whose mother's general health is excellent/very } \\
\text { good, good, or fair/poor. }\end{array}$ \\
\hline \multicolumn{2}{|l|}{ Notes for Data-Users } \\
\hline \multicolumn{2}{|c|}{$\begin{array}{l}\text { When the respondent to S9Q08 was the child's mother (biological, step, foster, adoptive), she rated her } \\
\text { own physical health status. Respondents who were not the child's mother (e.g., father or other relative) } \\
\text { gave a proxy rating of the mother's physical health. Nationally, about } 94 \text { percent of children had a } \\
\text { biological, step, foster, or adoptive mother in the household. }\end{array}$} \\
\hline \multicolumn{2}{|c|}{$\begin{array}{l}\text { Results for this question are weighted to reflect the population of children/youth ages } 0-17 \text { years, not } \\
\text { the population of parents! "Percent of children ages } 0-17 \text { years living with mothers whose health status } \\
\text { is excellent to poor" is the accurate way to report results - DO NOT report as "Percent of mothers...." }\end{array}$} \\
\hline \multicolumn{2}{|c|}{ SAS Code and Annotation } \\
\hline \multicolumn{2}{|l|}{ Format } \\
\hline \multicolumn{2}{|c|}{$\begin{array}{l}\text { value health /*ind1_1 ind6_1 ind6_2*/ } \\
\begin{aligned} \mathbf{1} & =\text { "Excellent/Very Good" } \\
\mathbf{2} & =\text { "Good" } \\
\mathbf{3} & =\text { "Fair/Poor"; }\end{aligned}\end{array}$} \\
\hline \multicolumn{2}{|l|}{ Code } \\
\hline \multicolumn{2}{|c|}{ 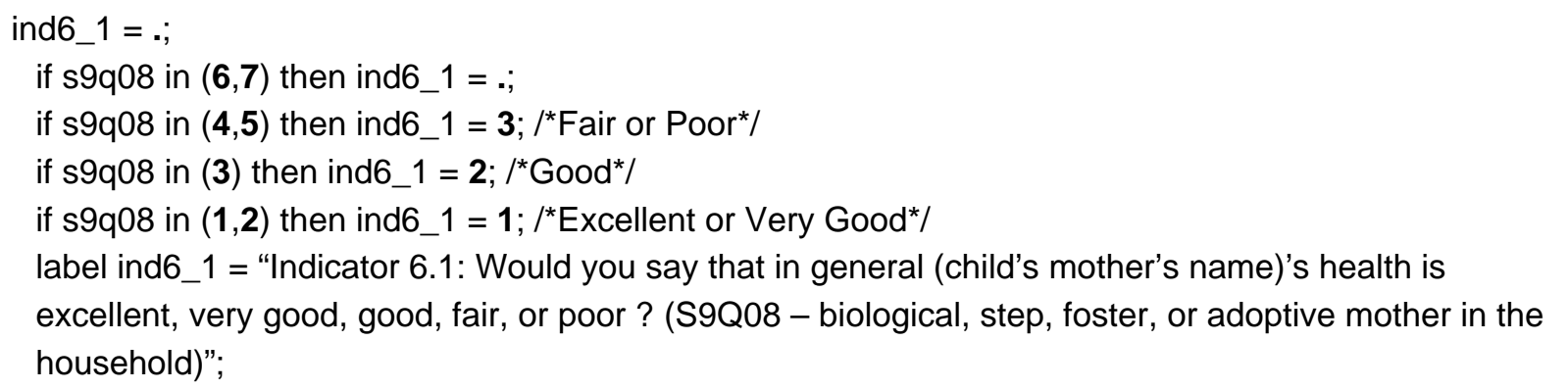 } \\
\hline
\end{tabular}




\begin{tabular}{|l|l|}
\hline INDICATOR 6.2 & $\begin{array}{l}\text { Would you say in general that (child's mother's name)'s mental and } \\
\text { emotional health is excellent, very good, good, fair, or poor? }\end{array}$ \\
\hline Survey Items Used & S9Q18 \\
\hline Numerators & $\begin{array}{l}\text { Children/youth whose mother's mental and emotional health is excellent or } \\
\text { very good }\end{array}$ \\
\hline $\begin{array}{l}\text { Children/youth whose mother's mental and emotional health is good } \\
\text { Children/youth whose mother's mental and emotional health is fair or poor }\end{array}$ \\
\hline Denominator & $\begin{array}{l}\text { Children/youth ages 0-17 years who live with their biological, step, foster or } \\
\text { adoptive mothers }\end{array}$ \\
\hline Description & $\begin{array}{l}\text { Percent of children/youth ages 0-17 whose mother's mental and emotional } \\
\text { health is excellent/very good, good, or fair/poor. }\end{array}$ \\
\hline Notes for Data-Users & \\
\hline
\end{tabular}

When the respondent to S9Q18 was the child's mother (biological, step, foster, adoptive), she rated her own mental/emotional health status. Respondents who were not the child's mother (e.g., father or other relative) gave a proxy rating of the mother's mental and emotional health. Nationally, about 94 percent of children had a biological, step, foster, or adoptive mother in the household.

Results for this question are weighted to reflect the population of children/youth ages 0-17 years, not the population of parents! "Percent of children/youth ages 0-17 years living with mothers whose mental health status is excellent to poor" is the accurate way to report results - DO NOT report as "Percent of mothers...."

\section{SAS Code and Annotation}

\section{Format}

value health /* ind1_1 ind6_1 ind6_2 */

$1=$ "Excellent/Very Good"

2 = "Good"

3 = "Fair/Poor";

\section{Code}

ind6_2 = .;

if s9q18 in $(6,7)$ then ind6_2 = ;

if s9q18 in $(\mathbf{4 , 5 )}$ then ind6_2 = 3; /*Fair or Poor*/

if s9q18 in (3) then ind6_2 $=2 ; /{ }^{*}$ Good $* /$

if s9q18 in $(\mathbf{1}, \mathbf{2})$ then ind6_2 = 1; /*Excellent or Very Good*/

label ind6_2 = "Indicator 6.2: Would you say in general that (child's mother's name)'s mental and emotional health is excellent, very good, good, fair, or poor? (S9Q18 - biological, step, foster, or adoptive mother in the household)"; 


\begin{tabular}{|c|c|}
\hline INDICATOR 6.3 & $\begin{array}{l}\text { Did child/youth have a mother whose physical and mental health were } \\
\text { BOTH excellent or very good? }\end{array}$ \\
\hline Survey Items Used & S9Q08 and S9Q18 \\
\hline Numerator & $\begin{array}{l}\text { Children/youth whose mother's overall physical and mental health status is } \\
\text { excellent or very good }\end{array}$ \\
\hline Denominator & $\begin{array}{l}\text { Children/youth ages 0-17 years who live with their biological, step, foster or } \\
\text { adoptive mothers }\end{array}$ \\
\hline Description & $\begin{array}{l}\text { Percent of children/youth ages 0-17 whose mother's physical and mental } \\
\text { health are BOTH excellent and very good (S9Q08 and S9Q18 both = } \\
\text { "excellent" or "very good"). }\end{array}$ \\
\hline \multicolumn{2}{|l|}{ Notes for Data-Users } \\
\hline \multicolumn{2}{|c|}{$\begin{array}{l}\text { When the respondent to S9Q08 or S9Q18 was the child's mother (biological, step, foster, adoptive) she } \\
\text { rated her own physical and mental health status. Respondents who were not the child's mother (e.g., } \\
\text { father or other relative) gave a proxy rating of the mother's physical or mental health. Nationally, about } \\
94 \text { percent of children had a biological, step, foster, or adoptive mother in the household. }\end{array}$} \\
\hline \multicolumn{2}{|c|}{$\begin{array}{l}\text { Results for this question are weighted to reflect the population of children/youth ages } 0-17 \text { years, not } \\
\text { the population of parents! "Percent of children/youth ages } 0-17 \text { years living with mothers whose overall } \\
\text { physical and mental health status is excellent or very good" is the accurate way to report results - DO } \\
\text { NOT report as "Percent of mothers...." }\end{array}$} \\
\hline \multicolumn{2}{|c|}{ SAS Code and Annotation } \\
\hline \multicolumn{2}{|l|}{ Format } \\
\hline \multicolumn{2}{|c|}{$\begin{array}{l}\text { value overall } / * \text { ind } 6 \_3 \text { */ } \\
\mathbf{1}=\text { = "One or both are NOT excellent/very good" } \\
\mathbf{2}=\text { "Maternal physical \& mental health BOTH excellent/very good"; }\end{array}$} \\
\hline \multicolumn{2}{|l|}{ Code } \\
\hline \multicolumn{2}{|c|}{ 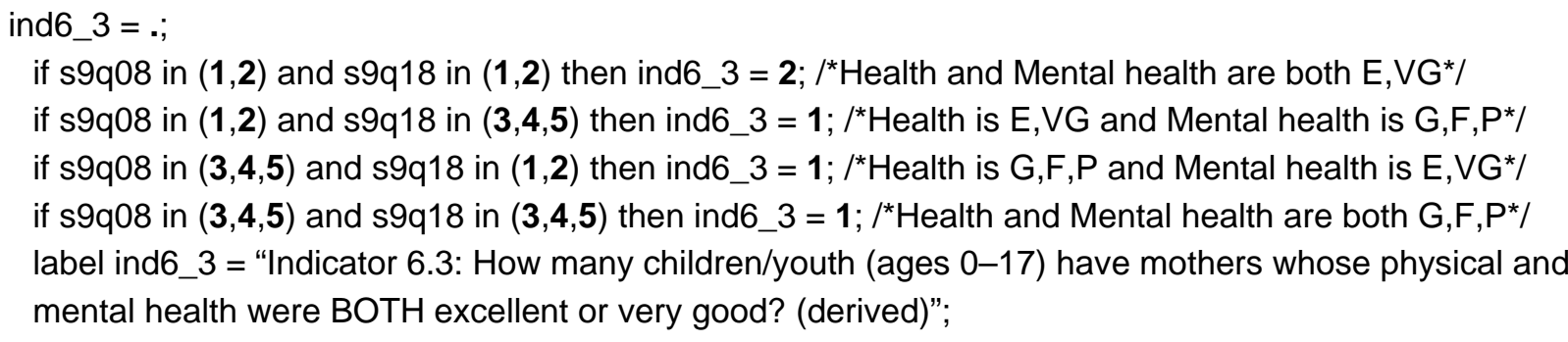 } \\
\hline
\end{tabular}




\begin{tabular}{|c|c|}
\hline INDICATOR 6.4 & Does anyone in the household use cigarettes, cigars, or pipe tobacco? \\
\hline Survey Items Used & S9Q11B \\
\hline Numerator & $\begin{array}{l}\text { Children/youth living in households where someone uses cigarettes, cigars, or } \\
\text { pipe tobacco }\end{array}$ \\
\hline Denominator & Children/youth ages 0-17 years \\
\hline Description & $\begin{array}{l}\text { Percent of children/youth ages 0-17 living in a household where someone uses } \\
\text { cigarettes, cigars, or pipe tobacco (S9Q11B = Yes). }\end{array}$ \\
\hline \multicolumn{2}{|l|}{ Notes for Data-Users } \\
\hline \multicolumn{2}{|c|}{$\begin{array}{l}\text { Missing data for S9Q11B includes } 13 \text { percent of respondents }(n=13,277) \text { who did not answer the } \\
\text { question because it was added to the } \mathrm{NSCH} \text { interview part way through the data collection process. }\end{array}$} \\
\hline \multicolumn{2}{|c|}{ SAS Code and Annotation } \\
\hline \multicolumn{2}{|l|}{ Format } \\
\hline \multicolumn{2}{|c|}{$\begin{array}{l}\text { value smoke /*ind6_4*/ } \\
\mathbf{1}=\text { "Someone in household smokes tobacco" } \\
\mathbf{2}=\text { "No one in household smokes tobacco"; }\end{array}$} \\
\hline \multicolumn{2}{|l|}{ Code } \\
\hline $\begin{array}{l}\text { ind6_4 = s9q11b; } \\
\text { if ind6_4 eq } 0 \text { then } \\
\text { if ind6_4 in }(6,7) \text { the } \\
\text { label ind6_4 = "Indi }\end{array}$ & $\begin{array}{l}6 \_4=2 \\
\text { nd6_4 = .; } \\
\text { or 6.4: Does anyone in the household use cigarettes, cigars, or pipe }\end{array}$ \\
\hline
\end{tabular}




\begin{tabular}{|c|c|}
\hline INDICATOR 6.5 & $\begin{array}{l}\text { How many children/youth live in "working poor" households - parents } \\
\text { employed full-time with incomes less than } 100 \text { percent of the Federal } \\
\text { Poverty Level (FPL)? }\end{array}$ \\
\hline Survey Items Used & S11Q08; C11Q01-W9Q12A \\
\hline Numerator & Children/youth living in "working poor" households \\
\hline Denominator & Children/youth ages 0-17 years \\
\hline Description & $\begin{array}{l}\text { Percent of children/youth ages 0-17 living in "working poor" households } \\
\text { (Parents employed full-time with incomes less than } 100 \text { percent of the FPL) }\end{array}$ \\
\hline \multicolumn{2}{|c|}{ Notes for Data-Users } \\
\hline \multicolumn{2}{|c|}{$\begin{array}{l}\text { "Working poor" is defined as a household in which one or both parents are employed full-time AND } \\
\text { household income is below the FPL. }\end{array}$} \\
\hline \multicolumn{2}{|c|}{ SAS Code and Annotation } \\
\hline \multicolumn{2}{|l|}{ Format } \\
\hline \multicolumn{2}{|c|}{$\begin{array}{l}\text { value workpoor } / * \text { ind6_5*/ } \\
\mathbf{1}=\text { ="Does not live in "working poor" HH" } \\
\mathbf{2}=\text { "Lives in "working poor" HH"; }\end{array}$} \\
\hline \multicolumn{2}{|l|}{ Code } \\
\hline \multicolumn{2}{|c|}{ 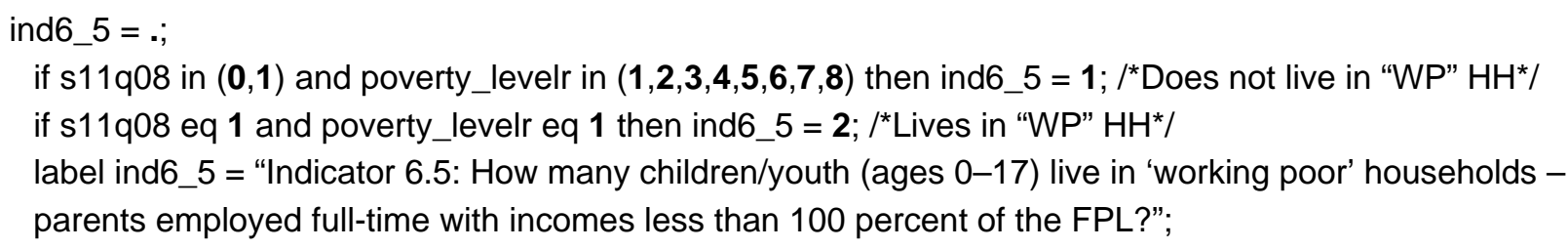 } \\
\hline
\end{tabular}




\begin{tabular}{|c|c|}
\hline INDICATOR 6.6 & $\begin{array}{l}\text { During the past week, how many times did you or any family member take } \\
\text { (child's name) on any kind of outing, such as to the park, library, zoo, } \\
\text { shopping, church, restaurants, or family gatherings? }\end{array}$ \\
\hline Survey Items Used & S8Q01 \\
\hline Numerators & $\begin{array}{l}\text { - Young children who went on no family outings the previous week } \\
\text { - Young children who went on 1-3 family outings the previous week } \\
\text { - Young children who went on 4-6 family outings the previous week } \\
\text { - } \quad \text { Young children who went on } 7 \text { or more family outings the previous week }\end{array}$ \\
\hline Denominator & Children ages $0-5$ years \\
\hline Description & $\begin{array}{l}\text { Percent of children ages } 0-5 \text { who went on outings with a family member no } \\
\text { times, } 1-3 \text { times, } 4-6 \text { times, or } 7 \text { or more times during the past week. }\end{array}$ \\
\hline \multicolumn{2}{|l|}{ Notes for Data-Users } \\
\hline \multicolumn{2}{|c|}{ SAS Code and Annotation } \\
\hline \multicolumn{2}{|l|}{ Format } \\
\hline \multicolumn{2}{|c|}{$\begin{array}{l}\text { value outings } / * \text { ind } 6 \_6 * / \\
\begin{aligned} \mathbf{1} & =\text { "None" } \\
\mathbf{2} & =\text { "1-3 times" } \\
\mathbf{3} & =\text { "4-6 times" } \\
\mathbf{4} & =\text { "7 or more times"; }\end{aligned}\end{array}$} \\
\hline \multicolumn{2}{|l|}{ Code } \\
\hline \multicolumn{2}{|c|}{ 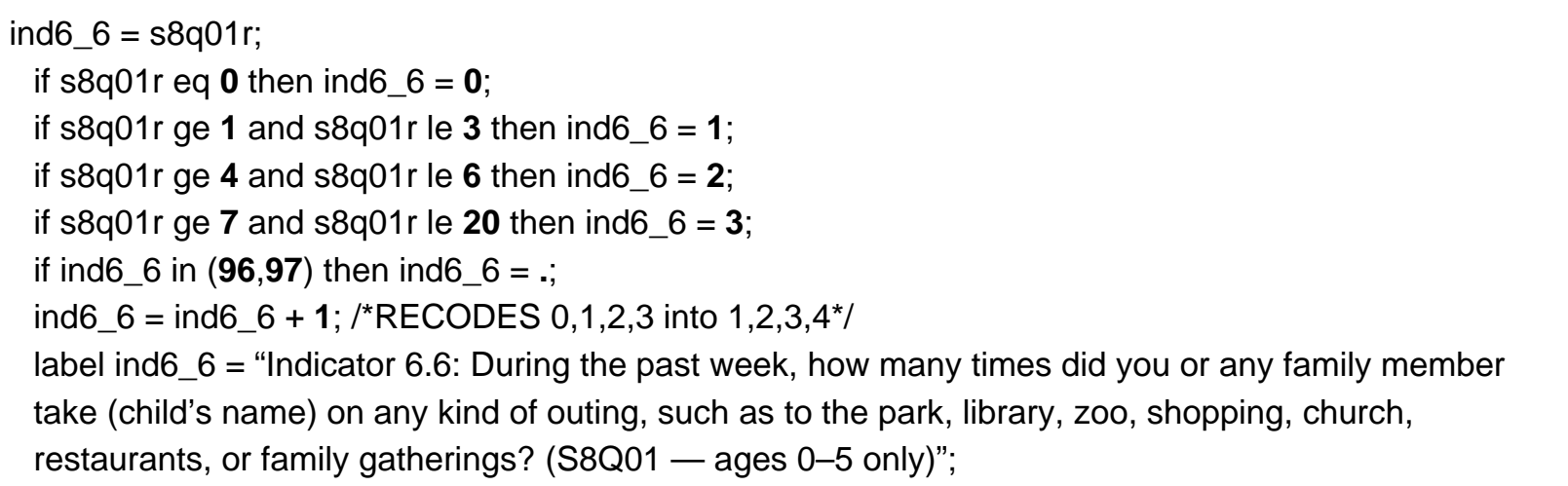 } \\
\hline
\end{tabular}




\begin{tabular}{|c|c|}
\hline INDICATOR 6.7 & $\begin{array}{l}\text { During the past week, how many days did you or other family members } \\
\text { read stories to (child's name)? }\end{array}$ \\
\hline Survey Items Used & S6Q62 \\
\hline Numerators & $\begin{array}{l}\text { - Young children who not read aloud to during the past week } \\
\text { - Young children who were read aloud to on 1-2 days during the past week } \\
\text { - } \quad \text { Young children who were read aloud to on 3-4 days during the past week } \\
\text { - Young children who were read aloud to on 5-6 days during the past week } \\
\text { - Young children who were read aloud to every day during the past week }\end{array}$ \\
\hline Denominator & Children ages $0-5$ years \\
\hline Description & $\begin{array}{l}\text { Percent of children ages } 0-5 \text { who were read aloud to by a family member on no } \\
\text { days, } 1-2 \text { days, 3-4 days, 5-6 days, or on everyday during the past week. }\end{array}$ \\
\hline \multicolumn{2}{|l|}{ Notes for Data-Users } \\
\hline \multicolumn{2}{|c|}{ SAS Code and Annotation } \\
\hline \multicolumn{2}{|l|}{ Format } \\
\hline $\begin{array}{l}\text { value reading /*ind6 } \\
\mathbf{1}=\text { = No days" } \\
\mathbf{2}=\text { = } 1 \text { or } 2 \text { days" } \\
\mathbf{3}=\text { = } 3 \text { or } 4 \text { days" } \\
\mathbf{4}=\text { = } 5 \text { or } 6 \text { days" } \\
\mathbf{5}=\text { "Every day"; }\end{array}$ & \\
\hline \multicolumn{2}{|l|}{ Code } \\
\hline \multicolumn{2}{|c|}{ 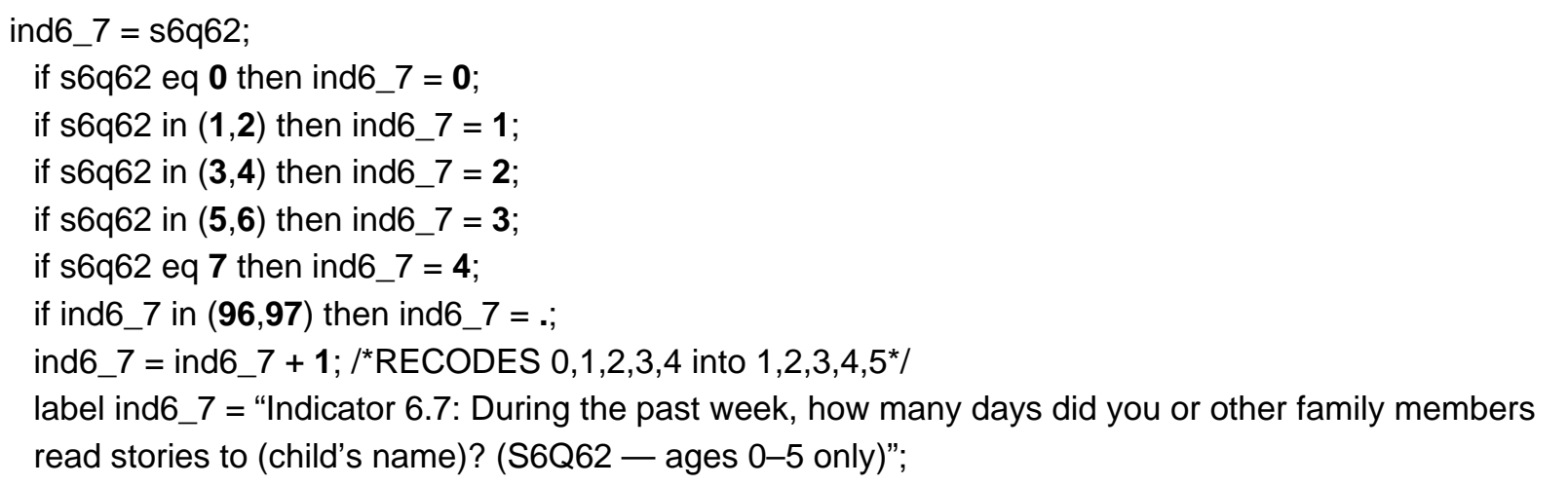 } \\
\hline
\end{tabular}




\begin{tabular}{|c|c|}
\hline INDICATOR 6.8 & $\begin{array}{l}\text { During the past week, on how many days did all the family members who } \\
\text { live in the household eat a meal together? }\end{array}$ \\
\hline Survey Items Used & S8Q03 \\
\hline Numerators & $\begin{array}{l}\text { - } \quad \text { Children/youth whose family did not eat together during the past week } \\
\text { - } \quad \text { Children/youth whose family ate together on 1-3 days during the past week } \\
\text { - } \quad \text { Children/youth whose family ate together on 4-6 days during the past week } \\
\text { - } \quad \text { Children/youth whose family ate together every day during the past week }\end{array}$ \\
\hline Denominator & Children/youth ages 0-17 years \\
\hline Description & $\begin{array}{l}\text { Percent of children/youth ages } 0-17 \text { live in a household where all of the family } \\
\text { members at a meat together on no days, } 1-3 \text { days, } 4-6 \text { days, or everyday } \\
\text { during the past week. }\end{array}$ \\
\hline \multicolumn{2}{|l|}{ Notes for Data-Users } \\
\hline \multicolumn{2}{|c|}{ SAS Code and Annotation } \\
\hline \multicolumn{2}{|l|}{ Format } \\
\hline $\begin{array}{l}\text { value shmeals /*ind6 } \\
\mathbf{1}=\text { "No days" } \\
\mathbf{2}=\text { "1-3 days" } \\
\mathbf{3}=\text { "4-6 days" } \\
\mathbf{4}=\text { "Every day"; }\end{array}$ & \\
\hline \multicolumn{2}{|l|}{ Code } \\
\hline $\begin{array}{l}\text { ind6_8 = s8q03; } \\
\text { if s8q03 eq } 0 \text { then i } \\
\text { if s8q03 ge } 1 \text { and s } \\
\text { if s8q03 ge } 4 \text { and s } \\
\text { if s8q03 eq } 7 \text { then i } \\
\text { if ind6_8 in }(96,97) \\
\text { ind6_8 = ind6_8+1 } \\
\text { label ind6_8 = "Indi } \\
\text { live in the househol }\end{array}$ & 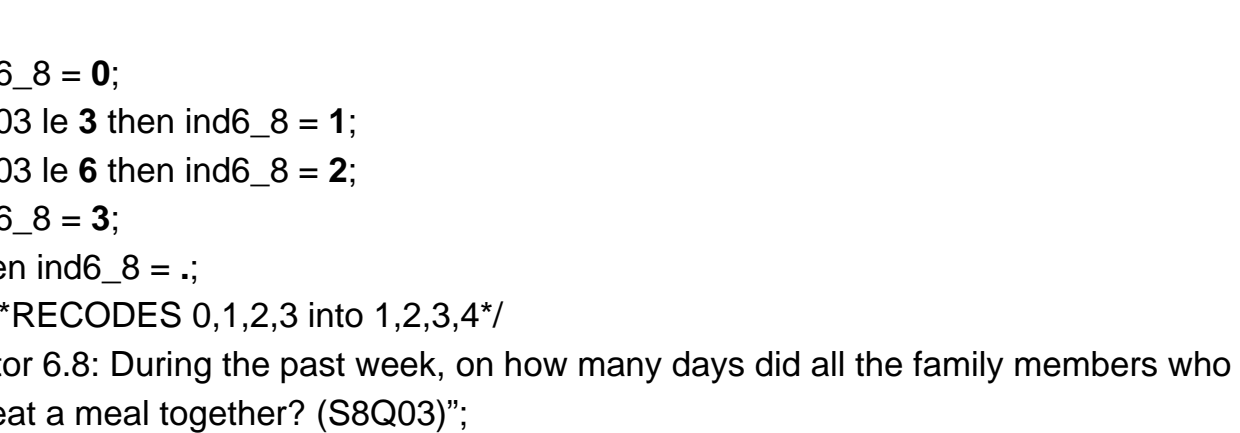 \\
\hline
\end{tabular}




\begin{tabular}{|c|c|}
\hline INDICATOR 6.9 & $\begin{array}{l}\text { During the past week, on how many nights did (child's name) get enough } \\
\text { sleep for a child/youth his/her age? }\end{array}$ \\
\hline Survey Items Used & S7Q20 \\
\hline Numerators & $\begin{array}{l}\text { - Children/youth who did not get enough sleep on any night during the past } \\
\text { - } \text { - Cheek } \\
\text { - Children/youth who got enough sleep on 1-3 nights during the past week } \\
\text { - Children/youth who got enough sleep on } 4-6 \text { nights during the past week } \\
\text { - } \text { got enough sleep on every night during the past week }\end{array}$ \\
\hline Denominator & Children/youth ages 6-17 years \\
\hline Description & $\begin{array}{l}\text { Percent of children/youth ages } 6-17 \text { who got enough sleep for a child of his/her } \\
\text { age on no nights, 1-3 nights, } 4-6 \text { nights, or every night during the past week. }\end{array}$ \\
\hline \multicolumn{2}{|l|}{ Notes for Data-Users } \\
\hline \multicolumn{2}{|c|}{ SAS Code and Annotation } \\
\hline \multicolumn{2}{|l|}{ Format } \\
\hline \multicolumn{2}{|l|}{$\begin{array}{l}\text { value sleep /*ind6_9*/ } \\
\begin{aligned} \mathbf{1} & =\text { "0 nights" } \\
\mathbf{2} & =\text { "1-3 nights" } \\
\mathbf{3} & =\text { "4-6 nights" } \\
\mathbf{4} & =\text { "Every night"; }\end{aligned}\end{array}$} \\
\hline \multicolumn{2}{|l|}{ Code } \\
\hline \multicolumn{2}{|c|}{ 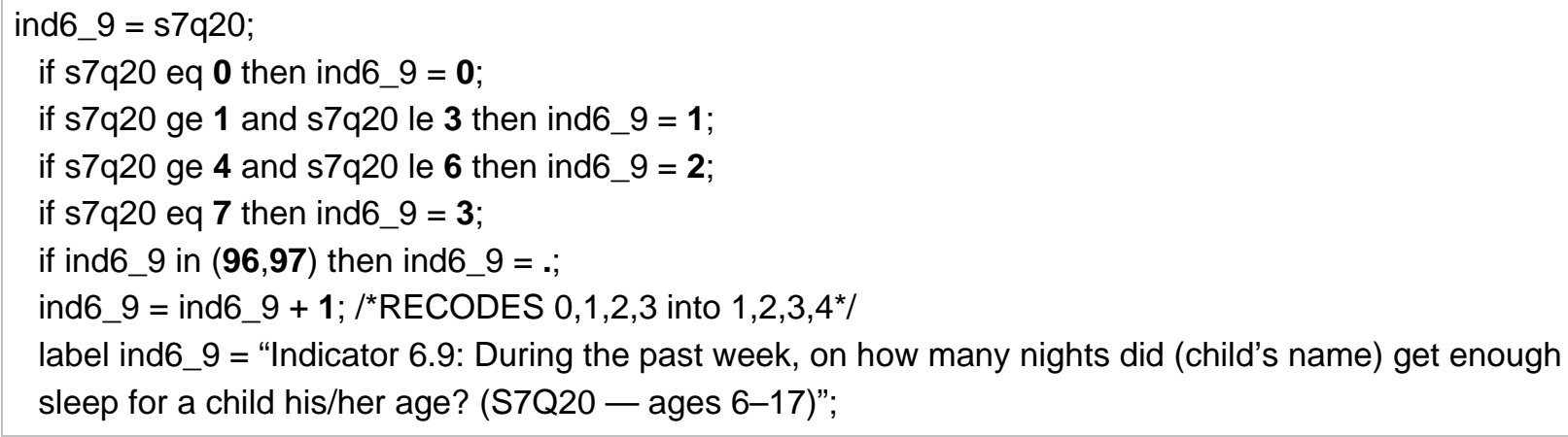 } \\
\hline
\end{tabular}




\begin{tabular}{|c|c|}
\hline INDICATOR 6.10 & $\begin{array}{l}\text { On an average school day, about how many hours does (child's name) } \\
\text { usually watch TV, watch videos, or play video games? }\end{array}$ \\
\hline Survey Items Used & S7Q28 \\
\hline Numerators & $\begin{array}{l}\text { - } \quad \text { Children/youth who do not spend time using TV/video on school days } \\
\text { - } \quad \text { Children/youth who spend an hour or less using TV/video on school days } \\
\text { - } \quad \text { Children/youth who spend } 2-3 \text { hours using TV/video on school days } \\
\text { - } \quad \text { Children/youth who spend } 4 \text { or more using TV/video on school days }\end{array}$ \\
\hline Denominator & Children/youth ages 6-17 years \\
\hline Description & $\begin{array}{l}\text { Percent of children/youth ages } 6-17 \text { who usually watch TV, watch videos, or } \\
\text { play video games for } 0 \text { hours, } 1 \text { hour or less, } 2-3 \text { hours, or } 4 \text { hours or more on } \\
\text { an average school day. }\end{array}$ \\
\hline \multicolumn{2}{|l|}{ Notes for Data-Users } \\
\hline \multicolumn{2}{|c|}{ Nationally, less than 0.5 percent of children/youth a } \\
\hline \multicolumn{2}{|c|}{ SAS Code and Annotation } \\
\hline \multicolumn{2}{|c|}{ Format } \\
\hline \multicolumn{2}{|c|}{$\begin{array}{l}\text { value tvwatch } / * \text { ind6_10*/ } \\
\begin{aligned} \mathbf{1} & =\text { "None" } \\
\mathbf{2} & =\text { "1 hour or less" } \\
\mathbf{3} & =\text { "2-3 hours" } \\
\mathbf{4} & =\text { "4 hours or more"; }\end{aligned}\end{array}$} \\
\hline \multicolumn{2}{|l|}{ Code } \\
\hline \multicolumn{2}{|c|}{ 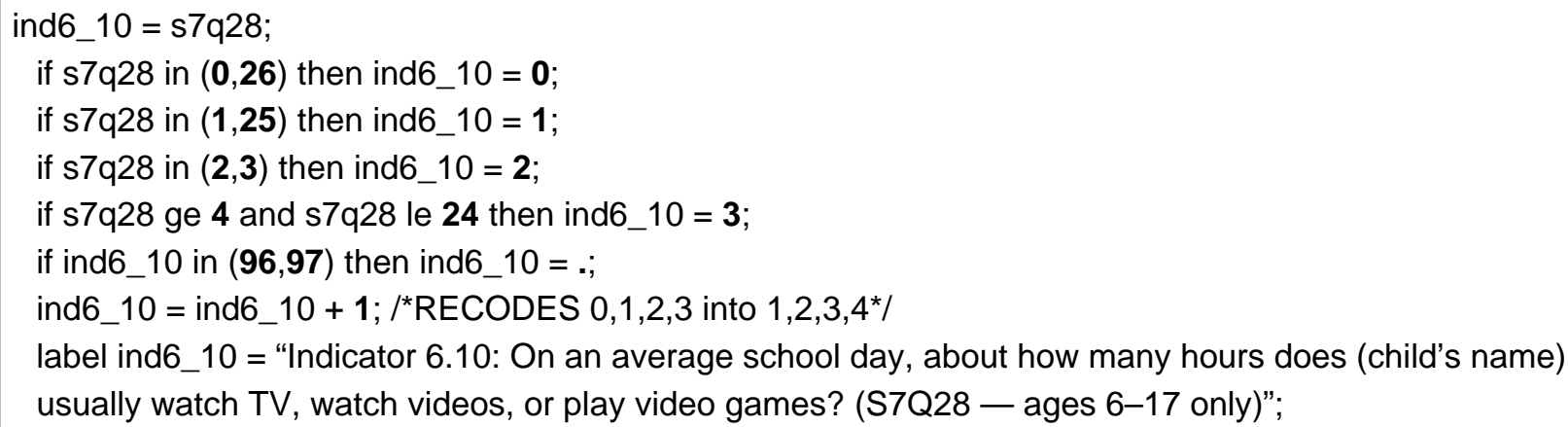 } \\
\hline
\end{tabular}




\begin{tabular}{|c|c|}
\hline INDICATOR 6.11 & $\begin{array}{l}\text { Sometimes children spend time caring for themselves at home without an } \\
\text { adult responsible for them. During the past week, did (child's name) } \\
\text { spend time caring for himself/herself for even a small amount of time? }\end{array}$ \\
\hline Survey Items Used & S7Q15 \\
\hline Numerator & Children who stayed alone at home for any length of time during past week \\
\hline Denominator & Children ages $6-11$ years \\
\hline Description & $\begin{array}{l}\text { Percent of children ages } 6-11 \text { who spent time caring for himself/herself for } \\
\text { even a small amount of time during the past week (S7Q15 = Yes). }\end{array}$ \\
\hline \multicolumn{2}{|c|}{ Notes for Data-Users } \\
\hline \multicolumn{2}{|c|}{ SAS Code and Annotation } \\
\hline \multicolumn{2}{|l|}{ Format } \\
\hline \multicolumn{2}{|c|}{$\begin{array}{l}\text { value ny /*ind6_11 ind6_14*/ } \\
\begin{aligned} \mathbf{1} & =\text { "No" } \\
\mathbf{2} & =\text { "Yes"; }\end{aligned}\end{array}$} \\
\hline \multicolumn{2}{|l|}{ Code } \\
\hline \multicolumn{2}{|c|}{$\begin{array}{l}\text { ind6_11 = s7q15; } \\
\text { if s7q15 eq } \mathbf{0} \text { then ind6_11= } \mathbf{1} \text {; } \\
\text { if s7q15 eq } \mathbf{1} \text { then ind6_11 = 2; } \\
\text { if ind6_11 in }(\mathbf{6}, 7) \text { then ind6_11 = .; } \\
\text { label ind6_11 = "Indicator 6.11: Sometimes children spend time caring for themselves at home without } \\
\text { an adult responsible for them. During the past week, did (child's name) spend time caring for } \\
\text { himself/herself for even a small amount of time? (S7Q15 - ages 6-11 only)"; }\end{array}$} \\
\hline
\end{tabular}




\section{INDICATOR 6.12 \\ Did child/youth have parents who often felt aggravated with them during the past month? \\ Survey Items Used \\ Numerator \\ Denominator \\ Description \\ S8Q07, S8Q08, S8Q10 \\ Children/youth whose parents report often feeling aggravated with child during past month \\ Children/youth ages 0-17 years \\ Percent of children/youth ages 0-17 living with parents who report often feeling aggravated with their child. \\ Notes for Data-Users}

"Often feeling aggravated" is parents who reported that during the last month, on average, they usually or always felt their child was much harder to care for than other children (S8Q07), or usually or always were bothered a lot by their child's behavior (S8Q08), or usually or always felt angry with their child (S8Q10).

S8Q07 - S8Q10 comprise the Aggravation in Parenting Scale, which was derived from the Parental Stress Index and the Parental Attitudes about Childrearing Scale. It has been used previously in the Panel Survey of Income Dynamics, the Survey of Income and Program Participation, and the Survey of Program Dynamics. Analysts should note that prior research revealed that the Aggravation in Parenting Scale has limited cultural validity among Spanish-speaking Latino parents. Removal of a single question (S8Q09) from the scale removed the measure for this group.

See www.cdc.gov/nchs/data/slaits/NSCH_Methodology_Report.pdf for more information.

\section{SAS Code and Annotation}

\section{Format}

value aggrav $/ *$ ind6_12*/

$1=$ "Parents seldom felt aggravated at child"

2 = "Parents often felt aggravated at child";

\section{Code}

array recode1 $\left\{{ }^{*}\right\}$ s8q07-s8q10; do $i=1$ to $\operatorname{dim}($ recode1); if recode1(i) in $(\mathbf{6}, \mathbf{7})$ then recode1(i)=.; else if recode $1(i)=\mathbf{1}$ then $\operatorname{recode} 1(i)=\mathbf{4}$; else if recode $1(i)=\mathbf{2}$ then $\operatorname{recode} 1(i)=\mathbf{3}$; else if recode1(i) $=\mathbf{3}$ then recode $1(i)=\mathbf{2}$; else if recode1(i) $=\mathbf{4}$ then recode1(i)=1; end; drop $\mathrm{i}$; test $=\mathrm{s} 8 \mathrm{q} 07^{*} \mathrm{~s} 8 \mathrm{q} 08^{*} \mathrm{~s} 8 \mathrm{q} 09^{*} \mathrm{~s} 8 \mathrm{q} 10 ;$ aggrav $=$.;

if test $>\mathbf{0}$ then aggrav $=(s 8 q 07+s 8 q 08+s 8 q 09+s 8 q 10) / 4$

else if s8q07 in (.,.M,.P) and s8q08 $>\mathbf{0}$ and s8q09 $>\mathbf{0}$ and s8q10 $>\mathbf{0}$ then aggrav $=(($ s8q08 + s8q09 + s8q10 $) / 3)$;

else if s8q08 in (.,.M,.P) and s8q07 > 0 and s8q09 $>\mathbf{0}$ and $s 8 q 10>\mathbf{0}$ then aggrav $=((s 8 q 07+s 8 q 09+s 8 q 10) / 3)$;

else if s8q09 in (.,.M,.P) and s8q07 $>\mathbf{0}$ and s8q08 $>\mathbf{0}$ and s8q10 $>\mathbf{0}$ then aggrav $=((s 8 q 07+s 8 q 08+s 8 q 10) / 3)$;

else if s8q10 in (.,.M,.P) and s8q07 >0 and s8q08 $>\mathbf{0}$ and s8q09 $>\mathbf{0}$ then aggrav $=((s 8 q 07+s 8 q 08+s 8 q 09) / 3)$; 


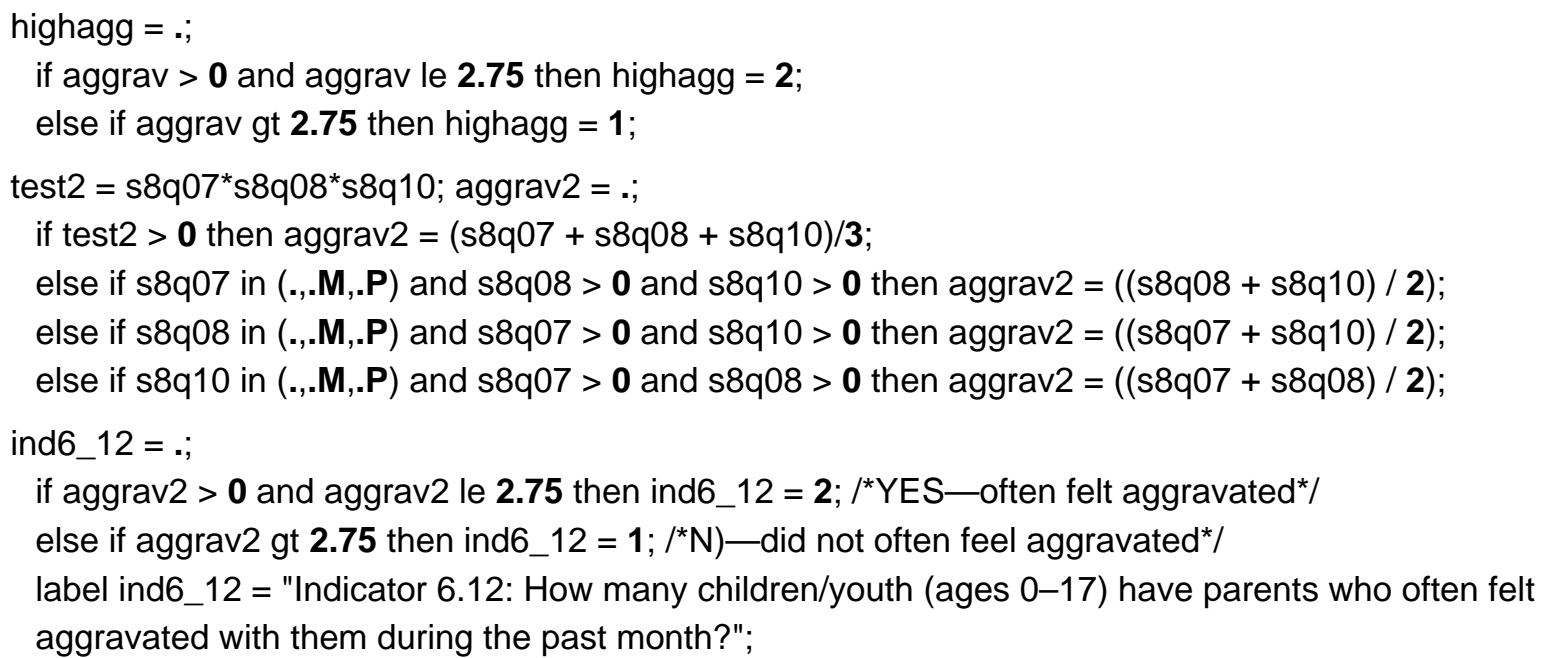
aggravated with them during the past month?"; 


\begin{tabular}{|c|c|}
\hline INDICATOR 6.13 & $\begin{array}{l}\text { During the past month, how many times have you had to make different } \\
\text { arrangements for child care at the last minute because your usual plans } \\
\text { changed due to circumstances beyond your control? }\end{array}$ \\
\hline Survey Items Used & S6Q53 \\
\hline Numerators & $\begin{array}{l}\text { - Young children whose parents did not need to make alternate child care } \\
\text { arrangements during the past month } \\
\text { - Young children whose parents had to make alternate child care } \\
\text { arrangements one time during the past month } \\
\text { - Young children whose parents had to make alternate child care } \\
\text { arrangements } 2-3 \text { times during the past month } \\
\text { - Young children whose parents had to make alternate child care } \\
\text { arrangements } 4 \text { or more times during the past month }\end{array}$ \\
\hline Denominator & Children ages $0-5$ years \\
\hline Description & $\begin{array}{l}\text { Percent of children ages } 0-5 \text { whose parent's made different child care } \\
\text { arrangements at the last minute no times, one time, } 2-3 \text { times, or } 4 \text { or more } \\
\text { times during the past month. }\end{array}$ \\
\hline
\end{tabular}

Notes for Data-Users

\section{SAS Code and Annotation}

\section{Format}

value lastmin $/ *$ ind6_13*/

$$
\begin{aligned}
& \mathbf{1}=\text { "None" } \\
& \mathbf{2}=\text { "1 time" } \\
& \mathbf{3}=\text { "2-3 times" } \\
& \mathbf{4}=\text { = } 4 \text { or more times"; }
\end{aligned}
$$

\section{Code}

ind6_13 = s6q53;

if s6q53 eq 0 then ind6_13 $=\mathbf{0}$;

if s6q53 eq 1 then ind6_13 = 1;

if s6q53 in $(2,3)$ then ind6_13 = 2 ;

if s6q53 ge $\mathbf{4}$ and s6q53 le 100 then ind6_13= $\mathbf{3}$;

if ind6_13 in $(995,996,997)$ then ind6_13 = .;

ind6_13 = ind6_13 + 1; /*RECODES 0,1,2,3 into 1,2,3,4*/

label ind6_13 = "Indicator 6.13: During the past month, how many times have you had to make different arrangements for child care at the last minute because your usual plans changed due to circumstances beyond your control? (S6Q53 - ages 0-5)"; 


\begin{tabular}{|c|c|}
\hline INDICATOR 6.14 & $\begin{array}{l}\text { During the past } 12 \text { months, did you or anyone in the family have to quit, } \\
\text { not take, or greatly change your job because of problems with child care } \\
\text { for (child's name)? }\end{array}$ \\
\hline Survey Items Used & S6Q54 \\
\hline Numerator & $\begin{array}{l}\text { Young children living with one or more family members whose employment was } \\
\text { affected by problems with child care during the past } 12 \text { months }\end{array}$ \\
\hline Denominator & Children ages $0-5$ years \\
\hline Description & $\begin{array}{l}\text { Percent of children ages } 0-5 \text { whose family members quit, did not take, or } \\
\text { greatly changed a job due to a child care problem during the past } 12 \text { months } \\
\text { (S6Q54 = Yes). }\end{array}$ \\
\hline \multicolumn{2}{|c|}{ Notes for Data-Users } \\
\hline \multicolumn{2}{|c|}{ SAS Code and Annotation } \\
\hline \multicolumn{2}{|l|}{ Format } \\
\hline \multicolumn{2}{|c|}{$\begin{array}{l}\text { value yn /*ind1_3 ind1_7 ind1_8 ind2_8 ind3_1 ind4_6 ind4_9 ind5_2 ind6_14*/ } \\
\mathbf{1}=\text { "Yes" } \\
\mathbf{2}=\text { "No"; }\end{array}$} \\
\hline \multicolumn{2}{|l|}{ Code } \\
\hline \multicolumn{2}{|c|}{$\begin{array}{l}\text { ind6_14 = s6q54; } \\
\text { if s6q54 eq } 1 \text { then ind6_14=1; } \\
\text { if s6q54 eq } \mathbf{0} \text { then ind6_14=0; } \\
\text { if ind6_14 in }(\mathbf{6}, 7) \text { then ind6_14 = ; } \\
\text { if ind6_14 = } \mathbf{0} \text { then ind6_14=2; /*RECODES } 0,1 \text { to } 1,2^{\star /} \\
\text { label ind6_14 = "Indicator } 6.14 \text { : During the past } 12 \text { months, did you or anyone in the family have to } \\
\text { quit, not take, or greatly change your job because of problems with child care for (child's name)? } \\
\text { (S6Q54 - ages } 0-5 \text { only)"; }\end{array}$} \\
\hline
\end{tabular}




\begin{tabular}{|c|c|}
\hline INDICATOR 6.15 & $\begin{array}{l}\text { Did child have parents who either had to make different child care } \\
\text { arrangements in the past month or employment-related changes due to } \\
\text { child care reasons in the past year or BOTH? }\end{array}$ \\
\hline Survey Items Used & S6Q53 and S6Q54 \\
\hline Numerator & Young children whose parents reported child care issues \\
\hline Denominator & Children ages $0-5$ years \\
\hline Interpretation & $\begin{array}{l}\text { Percent of children ages } 0-5 \text { years with one or more family members who } \\
\text { either quit, did not take, or greatly changes a job because of problems with } \\
\text { child care AND/OR children whose family had to make different child care plans } \\
\text { at the last minute (S6Q54 = Yes AND/OR S6Q53 = Yes). }\end{array}$ \\
\hline \multicolumn{2}{|c|}{ Notes for Data-Users } \\
\hline \multicolumn{2}{|c|}{$\begin{array}{l}\text { This indicator combines questions with different reference periods. Question S6Q53 asks about the } \\
\text { frequency of last minute changes in child care plans during the past month. Question S6Q54 asks about } \\
\text { employment-related issues during the past } 12 \text { months due to child care problems. }\end{array}$} \\
\hline \multicolumn{2}{|c|}{ SAS Code and Annotation } \\
\hline \multicolumn{2}{|l|}{ Format } \\
\hline \multicolumn{2}{|c|}{$\begin{array}{l}\text { value care_iss } / * \text { ind6_15*/ } \\
\mathbf{1}=\text { "One or more child care issues reported" } \\
\mathbf{2}=\text { "No child care issues reported"; }\end{array}$} \\
\hline \multicolumn{2}{|l|}{ Code } \\
\hline \multicolumn{2}{|c|}{ 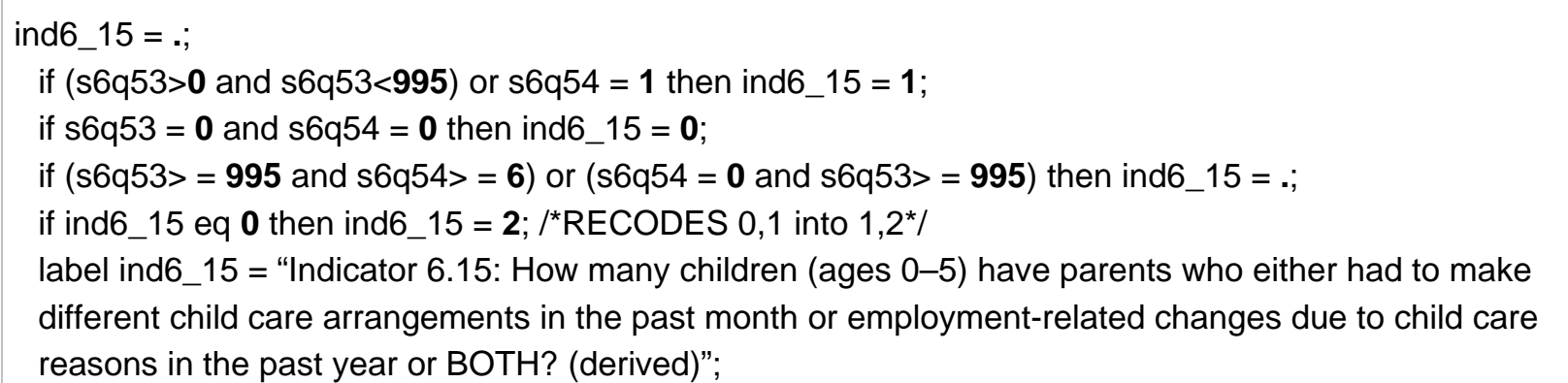 } \\
\hline
\end{tabular}




\begin{tabular}{|c|c|}
\hline INDICATOR 6.16 & $\begin{array}{l}\text { Did child regularly attend non-parental child care such as a child care } \\
\text { center, family-based center, in-home nanny, preschool, etc. during the } \\
\text { past month? }\end{array}$ \\
\hline Survey Items Used & S6Q48 - S6Q52 \\
\hline Numerator & Young children who regularly attend non-parental child care \\
\hline Denominator & Children ages $0-5$ years \\
\hline Interpretation & $\begin{array}{l}\text { Percent of children ages } 0-5 \text { years who regularly attended non-parental child } \\
\text { care in the past month. }\end{array}$ \\
\hline \multicolumn{2}{|c|}{ Notes for Data-Users } \\
\hline \multicolumn{2}{|c|}{$\begin{array}{l}\text { "Non-parental child care" is defined as one or more of the following: child care center, family-based child } \\
\text { care center, non-parental child care in home, nursery school, preschool, kindergarten, or Head or Early } \\
\text { Start. "Regular attendance" is defined as attending non-parental child care one or more times per week } \\
\text { during the month prior to the survey. }\end{array}$} \\
\hline \multicolumn{2}{|c|}{ SAS Code and Annotation } \\
\hline \multicolumn{2}{|l|}{ Format } \\
\hline \multicolumn{2}{|c|}{ 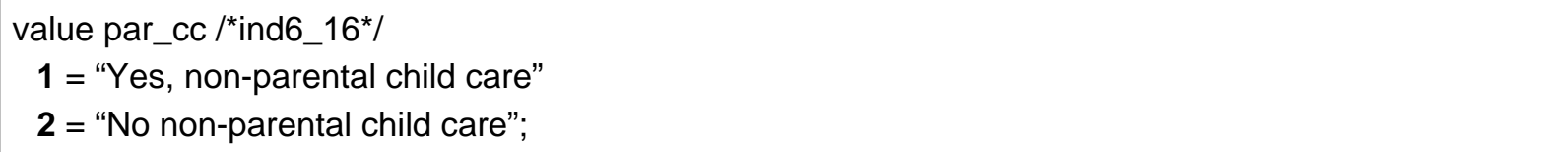 } \\
\hline \multicolumn{2}{|l|}{ Code } \\
\hline \multicolumn{2}{|c|}{ 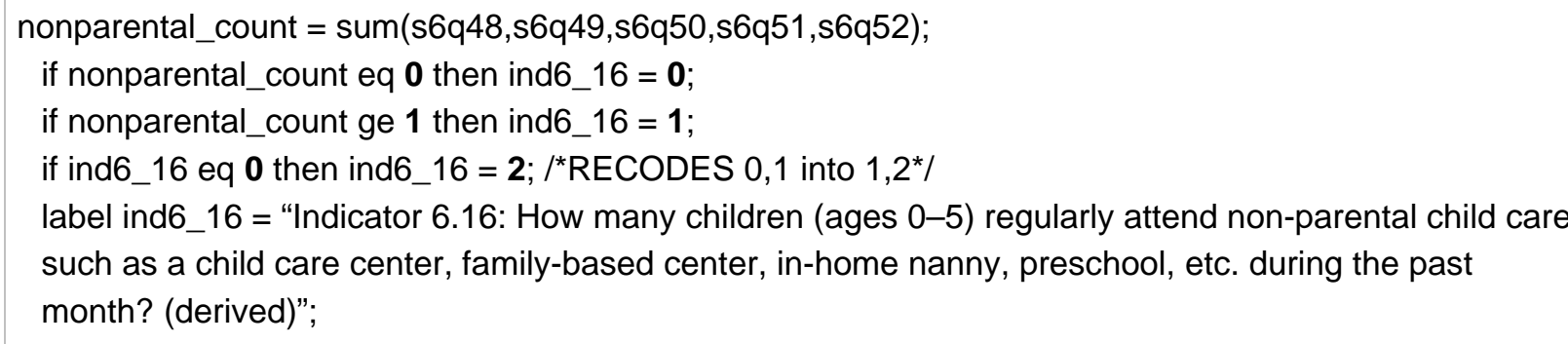 } \\
\hline
\end{tabular}




\begin{abstract}
INDICATOR 7.1 How many children/youth live in supportive neighborhoods?
Survey Items Used S10Q01 - S10Q03; S10Q05

Numerator

Children/youth living in supportive neighborhoods

Denominator

Children/youth ages 0-17 years

Interpretation

Percent of children/youth ages 0-17 who live in supportive neighborhoods (average score of less than 2.25 points across S10Q01 - S10Q03 and S10Q05.

Notes for Data-Users

S10Q01, S10Q02, S10Q03, and S10Q05 consider parents' perceived level of neighborhood social capital, focusing specifically on positive aspects of social capital relating to children. This concept, alternatively called social support, is similar to the concept of "social cohesion and trust," which is related to variations in violence among inner-city neighborhoods. These questions were originally developed for the Longitudinal Studies of Child Abuse and Neglect and have also been used for the Survey of Income and Program Participation.

See www.cdc.gov/nchs/data/slaits/NSCH_Methodology_Report.pdf for more information.

Scores are calculated for all children with valid responses to at least 3 of the 4 scale items. Response scoring: 1 = Definitely agree $2=$ Somewhat agree $3=$ Somewhat disagree $4=$ Definitely disagree.

Cutoff score of less than 2.25 indicates one or fewer responses of "somewhat disagree" or "definitely disagree" across scale items.
\end{abstract}

\title{
SAS Code and Annotation
}

\section{Format}

value suppnhbd /*ind7_1*I

$\mathbf{1}=$ "Do not live in a supportive neighborhood"

$\mathbf{2}$ = "Live in a supportive neighborhood";

\section{Code}

ind7_1 = ;

if s10q01 in $(6,7)$ then $\mathrm{s} 10 \mathrm{q} 01=$;

if $\mathrm{s} 10 \mathrm{q0} 2 \mathrm{in}(6,7)$ then $\mathrm{s} 10 \mathrm{q0}=$; ;

if $\mathrm{s} 10 \mathrm{q} 03$ in $(6,7)$ then $\mathrm{s} 10 \mathrm{q} 03=$;;

if $\mathrm{s} 10 \mathrm{q} 05$ in $(\mathbf{6}, \mathbf{7})$ then $\mathrm{s} 10 \mathrm{q} 05=$;;

if $s 10 q 01$ ge 1 then $s 10 q 01 \mathrm{c}=1$;

if $s 10 q 02$ ge 1 then $s 10 q 02 c=1$;

if $s 10 q 03$ ge 1 then $s 10 q 03 c=1$;

if $s 10 q 05$ ge 1 then $s 10 q 05 c=1$;

tot_supp = sum(s10q01c,s10q02c,s10q03c,s10q05c);

if tot_supp ge 3 then do;

supp_nbhd = mean(s10q01,s10q02,s10q03,s10q05);end;

label supp_nbhd = "Supportive neighborhood mean score on 3 of 4 component variables";

if supp_nbhd ge 0 and supp_nbhd le 2.20 then ind7_1 = 0; 
if supp_nbhd ge 2.25 and supp_nbhd le 4.00 then ind7_1 = 1;

if ind7_1 eq 0 then ind7_1 = 2; /*RECODES 0,1 into $1,2 *$ I

label ind7_1 = "Indicator 7.1: How many children/youth (ages 0-17) live in supportive neighborhoods? (derived)"; 


\begin{tabular}{|c|c|}
\hline INDICATOR 7.2 & $\begin{array}{l}\text { How often do you feel (child's name) is safe in your community or } \\
\text { neighborhood? }\end{array}$ \\
\hline Survey Items Used & S10Q06 \\
\hline Numerators & $\begin{array}{l}\text { - Children/youth whose parents feel they are usually or always safe in their } \\
\text { neighborhood } \\
\text { - Children/youth whose parents feel they are sometimes safe in their } \\
\text { neighborhood } \\
\text { - Children/youth whose parents feel they are never safe in their } \\
\text { neighborhood }\end{array}$ \\
\hline Denominator & Children/youth ages 0-17 years \\
\hline Interpretation & $\begin{array}{l}\text { Percent of children/youth ages } 0-17 \text { whose parents feel they are usually or } \\
\text { always, sometimes, or never safe in their community or neighborhood. }\end{array}$ \\
\hline \multicolumn{2}{|l|}{ Notes for Data-Users } \\
\hline \multicolumn{2}{|c|}{$\begin{array}{l}\text { Results for this question are weighted to reflect the population of children/youth ages } 0-17 \text { years, not } \\
\text { the population of parents or family members! "Percent of children/youth ages } 0-17 \text { years whose parents } \\
\text { feel that the child is always safe in their community or neighborhood" is the accurate way to report } \\
\text { results - DO NOT report as "Percent of parents...." }\end{array}$} \\
\hline \multicolumn{2}{|c|}{ SAS Code and Annotation } \\
\hline \multicolumn{2}{|l|}{ Format } \\
\hline \multicolumn{2}{|c|}{$\begin{array}{l}\text { value safe /*ind7_2 ind7_3*/ } \\
\mathbf{1}=\text { "Usually or always safe" } \\
\mathbf{2}=\text { "Sometimes safe" } \\
\mathbf{3}=\text { "Never safe"; }\end{array}$} \\
\hline \multicolumn{2}{|l|}{ Code } \\
\hline \multicolumn{2}{|c|}{ 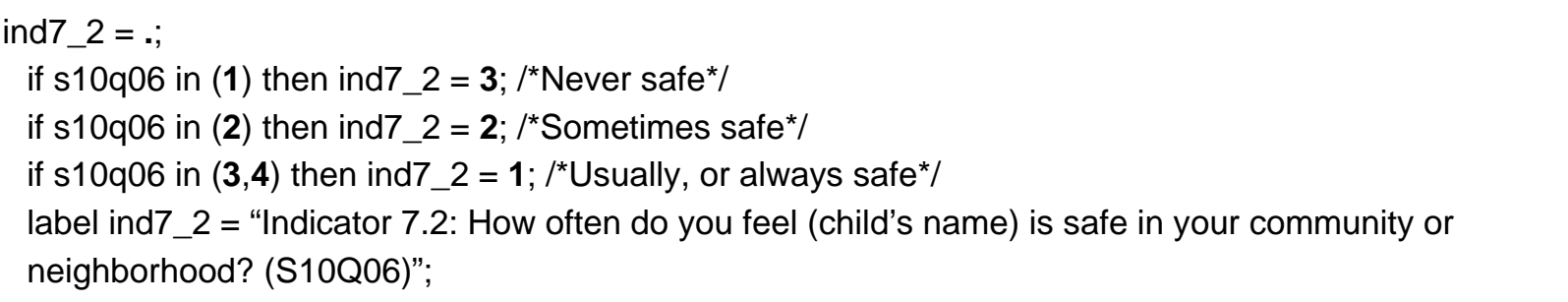 } \\
\hline
\end{tabular}




\begin{tabular}{|c|c|}
\hline INDICATOR 7.3 & How often do you feel (child's name) is safe at school? \\
\hline Survey Items Used & S10Q07 \\
\hline Numerators & $\begin{array}{l}\text { - Children/youth whose parents feel they are usually or always safe at school } \\
\text { - } \quad \text { Children/youth whose parents feel they are sometimes at school } \\
\text { - } \quad \text { Children/youth whose parents feel they are never safe at school }\end{array}$ \\
\hline Denominator & Children/youth ages 6-17 years \\
\hline Interpretation & $\begin{array}{l}\text { Percent of children/youth ages } 0-17 \text { whose parents feel they are usually or } \\
\text { always, sometimes, or never safe at school. }\end{array}$ \\
\hline \multicolumn{2}{|l|}{ Notes for Data-Users } \\
\hline \multicolumn{2}{|c|}{$\begin{array}{l}\text { Results for this question are weighted to reflect the population of children ages } 6-17 \text { years, not the } \\
\text { population of parents or family members. "Percent of children ages } 6-17 \text { years whose parents feel that } \\
\text { the child is always safe at school" is the accurate way to report results - DO NOT report as "Percent of } \\
\text { parents..." }\end{array}$} \\
\hline \multicolumn{2}{|c|}{ SAS Code and Annotation } \\
\hline \multicolumn{2}{|l|}{ Format } \\
\hline \multicolumn{2}{|c|}{$\begin{array}{l}\text { value safe } / * \text { ind7_2 ind7_3*I } \\
\mathbf{1}=\text { "Usually or always safe" } \\
\mathbf{2}=\text { "Sometimes safe" } \\
\mathbf{3}=\text { "Never safe"; }\end{array}$} \\
\hline \multicolumn{2}{|l|}{ Code } \\
\hline \multicolumn{2}{|c|}{ 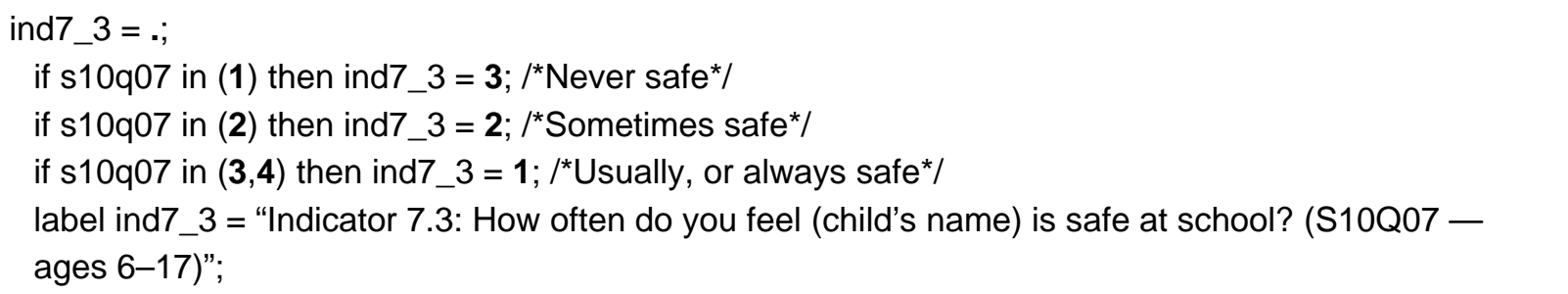 } \\
\hline
\end{tabular}




\begin{tabular}{|c|c|}
\hline Gender & How many males and females are in the 0 - to 17 -year-old population? \\
\hline Survey Items Used & S1Q01 \\
\hline Numerators & $\begin{array}{ll}\text { - } & \text { Male } \\
\text { - } & \text { Female }\end{array}$ \\
\hline Denominator & Children/youth ages $0-17$ years \\
\hline Interpretation & Percent of children/youth ages $0-17$ who are male or female. \\
\hline \multicolumn{2}{|l|}{ Notes for Data-Users } \\
\hline \multicolumn{2}{|c|}{ SAS Code and Annotation } \\
\hline \multicolumn{2}{|l|}{ Format } \\
\hline \multicolumn{2}{|l|}{$\begin{array}{l}\text { value sex } \\
\qquad \begin{array}{l}\mathbf{1}=\text { "Male" } \\
\mathbf{2}=\text { "Female"; }\end{array}\end{array}$} \\
\hline \multicolumn{2}{|l|}{ Code } \\
\hline $\begin{array}{l}\text { sex = s1q01; } \\
\text { if s1q01 in }(6,7) \text { the } \\
\text { label sex = "How me }\end{array}$ & $\begin{array}{l}x=; \\
\text { nales and females are in the 0- to 17-year-old population? (S1Q01)"; }\end{array}$ \\
\hline
\end{tabular}




\begin{tabular}{|c|c|}
\hline Age Group (3) & $\begin{array}{l}\text { How many children/youth in the } 0 \text { - to } 17 \text {-year-old population are in each } \\
\text { of three age groups? }\end{array}$ \\
\hline Survey Items Used & Initial sampling questions \\
\hline Numerators & $\begin{array}{ll}- & 0-5 \text { years old } \\
\text { - } & 6-11 \text { years old } \\
\text { - } & 12-17 \text { years old }\end{array}$ \\
\hline Denominator & Children/youth ages 0-17 years \\
\hline Interpretation & Percent of children/youth who are $0-5,6-11$, or $12-17$. \\
\hline \multicolumn{2}{|l|}{ Notes for Data-Users } \\
\hline \multicolumn{2}{|c|}{ SAS Code and Annotation } \\
\hline \multicolumn{2}{|l|}{ Format } \\
\hline $\begin{array}{l}\text { value agethree } \\
\begin{aligned} \mathbf{1} & =\text { "0-5 yrs old" } \\
\mathbf{2} & =\text { "6-11 yrs old" } \\
\mathbf{3} & =\text { "12-17 yrs old"; }\end{aligned}\end{array}$ & \\
\hline \multicolumn{2}{|l|}{ Code } \\
\hline $\begin{array}{l}\text { age_3 }=\text { AGEYR_CHI } \\
\text { if AGEYR_CHILD ge } \\
\text { if AGEYR_CHILD ge } \\
\text { if AGEYR_CHILD ge } \\
\text { label age_3 }=\text { "How } \\
\text { (derived_ } 3 \text { groups }\end{array}$ & $\begin{array}{l}\text { and AGEYR_CHILD le } \mathbf{5} \text { then age_3 }=\mathbf{1} ; \\
\text { and AGEYR_CHILD le } \mathbf{1 1} \text { then age_3 }=\mathbf{2} \text {; } \\
\text { and AGEYR_CHILD le } \mathbf{1 7} \text { then age_3=3; } \\
\text { ny children/youth of different ages are in the } 0 \text { - to } 17 \text {-year-old population? }\end{array}$ \\
\hline
\end{tabular}




\begin{tabular}{|c|c|}
\hline Age Group (5) & $\begin{array}{l}\text { How many children/youth in the } 0 \text { - to } 17-\text { year-old population are in each } \\
\text { of five age groups? }\end{array}$ \\
\hline Survey Items Used & Initial sampling questions \\
\hline Numerators & $\begin{array}{ll}\text { - } & 0-3 \text { years old } \\
\text { - } & 4-7 \text { years old } \\
\text { - } & \text { 8-11 years old } \\
\text { - } & 12-14 \text { years old } \\
\text { - } & 15-17 \text { years old }\end{array}$ \\
\hline Denominator & Children/youth ages $0-17$ years \\
\hline Interpretation & Percent of children/youth who are $0-3,4-7,8-11,12-14$, or $15-17$. \\
\hline \multicolumn{2}{|l|}{ Notes for Data-Users } \\
\hline \multicolumn{2}{|c|}{ SAS Code and Annotation } \\
\hline \multicolumn{2}{|l|}{ Format } \\
\hline $\begin{array}{l}\text { value agefive } \\
\begin{aligned} \mathbf{1} & =\text { "0-3 yrs old" } \\
\mathbf{2} & =\text { "4-7yrs old" } \\
\mathbf{3} & =\text { "8-11 yrs old" } \\
\mathbf{4} & =\text { "12-14 yrs old" } \\
\mathbf{5} & =\text { "15-17 yrs old"; }\end{aligned}\end{array}$ & \\
\hline \multicolumn{2}{|l|}{ Code } \\
\hline $\begin{array}{l}\text { age_5 = AGEYR_CH } \\
\text { if AGEYR_CHILD g } \\
\text { if AGEYR_CHILD g } \\
\text { if AGEYR_CHILD g } \\
\text { if AGEYR_CHILD g } \\
\text { if AGEYR_CHILD g }\end{array}$ & $\begin{array}{l}\text { and AGEYR_CHILD le } 3 \text { then age_5 = } \mathbf{1} \\
\text { and AGEYR_CHILD le } 7 \text { then age_5 = 2; } \\
\text { and AGEYR_CHILD le } 11 \text { then age_5 = 3; } \\
\text { and AGEYR_CHILD le } 14 \text { then age_5 }=\mathbf{4} ; \\
\text { and AGEYR_CHILD le } 17 \text { then age_5 }=\mathbf{5}\end{array}$ \\
\hline $\begin{array}{l}\text { label age_ } 5=\text { "How } \\
\text { (derived }-5 \text { groups }\end{array}$ & $y$ children/youth of different ages are in the 0 - to 17 -year-old population? \\
\hline
\end{tabular}




\begin{tabular}{|c|c|}
\hline Race and Ethnicity & $\begin{array}{l}\text { How many children of different races or Hispanic ethnicity are in the } \\
0 \text { - to } 17 \text {-year-old population? }\end{array}$ \\
\hline Survey Items Used & S11Q01; S11Q02X01 - S11Q02X07 \\
\hline Numerators & $\begin{array}{ll}\text { - } & \text { Hispanic } \\
\text { - } & \text { White, non-Hispanic } \\
\text { - } & \text { Black, non-Hispanic } \\
\text { - } & \text { Multiracial, non-Hispanic } \\
\text { - } & \text { Other, non-Hispanic }\end{array}$ \\
\hline Denominator & Children/youth ages 0-17 years \\
\hline Interpretation & $\begin{array}{l}\text { Percent of children/youth ages 0-17 who are Hispanic/Latino, or non- } \\
\text { Hispanic and white, black, multiracial, or of another race. }\end{array}$ \\
\hline \multicolumn{2}{|l|}{ Notes for Data Users } \\
\hline \multicolumn{2}{|c|}{$\begin{array}{l}\text { Children/youth reporting Hispanic or Latino ethnicity (S11Q01 = Yes) are grouped as HISPANIC, } \\
\text { regardless of race. Non-Hispanic children are grouped by race based on responses to S11Q02X01 - } \\
\text { S11Q02X07. Non-Hispanic children reporting more than one race category are grouped as "MULTI- } \\
\text { RACE". At the national level, non-Hispanic children reporting only one race category of Asian, American } \\
\text { Indian, Alaska Native, Native Hawaiian, or Pacific Islander are combined into a single group called } \\
\text { OTHER. This is done because only a handful of States meet the NCHS' confidentiality standards for } \\
\text { releasing data on these minority groups to the public. NCHS only includes individual level data for one } \\
\text { of these minority groups in the publicly available data files when a group comprises } 5 \text { percent or more } \\
\text { of the total child population in a State. This standard is in place to protect against the remote chance of } \\
\text { an individual child inadvertently being identified in States with relatively few children from these minority } \\
\text { groups in their populations. For more information, see } \\
\text { www.cdc.gov/nchs/data/slaits/NSCH Methodology Report.pdf }\end{array}$} \\
\hline
\end{tabular}

\section{SAS Code and Annotation}

\section{Format}

\section{value race}

$\mathbf{1}=$ "Hispanic"

2 = "White, non-Hispanic"

3 = "Black, non-Hispanic"

$4=$ "Multiracial, non-Hispanic"

$\mathbf{5}$ = "Other, non-Hispanic";

\section{Code}

race_5 = .;

if racer eq 1 then race_ $5=2$;

if racer eq 2 then race_ $5=\mathbf{3}$;

if racer eq 3 then race_ $5=4$;

if racer eq $\mathbf{4}$ then race_ $5=\mathbf{5}$;

if s11q01 eq 1 then race_ $5=\mathbf{1}$;

if racer in $(6,7)$ then race_ $5=$.; 
Stratifiers

label race_5 = "How many children of different races or Hispanic ethnicity are in the 0- to 17-year-old population? (derived)"; 


\begin{abstract}
Special Health Care How many children/youth have special health care needs?
Needs Status

Survey Items Used $\quad$ S2Q04 - S2Q17

Numerator Children/youth with qualifying responses on one or more of the five CSHCN

Screener criteria

Denominator

Children/youth ages 0-17 years

Interpretation

Percent of children/youth ages 0-17 with qualifying responses on one or more of the five CSHCN Screener criteria.

Notes for Data Users

Questions S2Q04 - S2Q17 comprise the CSHCN Screener, a validated screening instrument for identifying children with special health care needs as defined by the federal Maternal and Child Health Bureau. The CSHCN Screener asks whether a child currently experiences any of five different health consequences, and if so, whether that specific health consequence is due to a medical, behavioral, or other type of health condition that has lasted or is expected to last 12 months or longer. A child must have affirmative responses on all 3 parts (or 2 parts in the case of screening question for the ongoing emotional, developmental, or behavioral conditions criteria) of a screening question in order to qualify on that particular screening criteria.

For more information on the development, testing, and scoring of the CSHCN Screener, email CAHMI@ohsu.edu. For more information on use of the CSHCN screener in the $2003 \mathrm{NSCH}$, see www.cdc.gov/nchs/data/slaits/NSCH_Methodology_Report.pdf.

Step 1 in the code below creates yes-no variables identifying children with qualifying responses to each of the five different CSHCN Screener criteria. Step 2 creates the summary variable that indicates whether or not the child/youth has special health care needs based on having qualifying responses to one or more of the five screening criteria. Step 3 of the code creates two optional analytic variables that can be used to subclassify CSHCN into mutually exclusive groups based on number and type of special health care needs.
\end{abstract}

\title{
SAS Code and Annotation
}

\section{Format}

value metlwi

$1=$ "CSHCN"

2 = "Non-CSHCN";

/* formats below are for screener and optional analytical variables *

value ny /*used for screener items: rxmeds, serve, func, therapy, mhealth*/

$\mathbf{0}=$ "No";

$1=$ "Yes"

value Iwiscren

$1=$ "Non-CSHCN"

2 = "One qualifying answer"

$\mathbf{3}=$ "Two qualifying answers" 


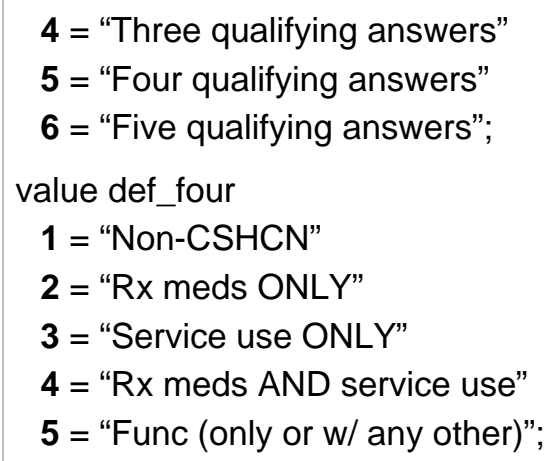

Code

I**STEP 1: Create interim variables that identify children with qualifying responses on each of the five different CSHCN Screener criteria. These five variables are also valuable analytic tools for further studying the group of children identified by the CSHCN Screener *I

rxmeds $=0$;

if $((\mathrm{S} 2 \mathrm{Q} 04=1)$ and $(\mathrm{S} 2 \mathrm{Q} 05=1)$ and $(\mathrm{S} 2 \mathrm{Q} 06=1))$ then rxmeds = rxmeds + 1; label rxmeds = "Qualified on rx med use?";

serve = 0; if $((\mathrm{S} 2 \mathrm{Q} 07=1)$ and $(\mathrm{S} 2 \mathrm{Q} 08=1)$ and $(\mathrm{S} 2 \mathrm{Q} 09=1))$ then serve = serve +1 ; label serve = "Qualified on service use?";

func $=0$;

if $((\mathrm{S} 2 \mathrm{Q} 10=1)$ and $(\mathrm{S} 2 \mathrm{Q} 11=1)$ and $(\mathrm{S} 2 \mathrm{Q} 12=1))$ then func $=$ func +1 ;

label func = "Qualified on functional limits?";

therapy $=0$;

if $((\mathrm{S} 2 \mathrm{Q} 13=1)$ and $(\mathrm{S} 2 \mathrm{Q} 14=1)$ and $(\mathrm{S} 2 \mathrm{Q} 15=1))$ then therapy = therapy +1 ;

label therapy = "Qualified on use of special therapies?";

mhealth $=0$;

if $((\mathrm{S} 2 \mathrm{Q} 16=1)$ and $(\mathrm{S} 2 \mathrm{Q} 17=1))$ then mhealth = mhealth +1 ;

label mhealth = "Qualified on use of mental health services?";

$1 * \star$ STEP 2: Compute summary CSHCN Screener score identifying children with 1 or more qualifying responses to any of the 5 screening criteria**

metlwi = 0;

if $($ rxmeds $=1)$ or $($ serve $=1)$ or $($ func $=1)$ or $($ therapy $=1)$ or $($ mhealth $=1)$ then metlwi $=1$;

if metlwi $=0$ then metlwi $=2 ; /{ }^{*}$ RECODES 0,1 into $1,2^{\star} /$

label metlwi = "Results for CSHCN screener";

/**STEP 3 (OPTIONAL): Compute variables useful for sub-classifying the CSHCN Group based on quantitative and qualitative schemes ${ }^{\star * /}$

/**Mutually exclusive quantitative subgrouping of special health care need types - based on number of qualifying screening criteria ${ }^{\star \star} /$

Iwiscren = sum (rxmeds, serve, func, therapy, mhealth);

Iwiscren $=$ Iwiscren $+1 ;$ / RECODES 0 thru 5 into 1 thru $6 *$ I 
label Iwiscren = "CSHCN grouped according to number of qualifying screener answers";

/**Mutually exclusive qualitative subgrouping of special health care need types - based on type of qualifying screening criteria ${ }^{\star \star} /$

def_4 = 0;

if (func $=0)$ and $(($ rxmeds $=1))$ and $(($ serve $=0)$ or (therapy $=0)$ or $($ mhealth $=0))$ then def_4 $=1$; if $($ func $=0)$ and $(($ rxmeds $=0))$ and $(($ serve $=1)$ or (therapy $=1)$ or $($ mhealth $=1))$ then def_4 $=2$; if $($ func $=0)$ and $(($ rxmeds $=1))$ and $(($ serve $=1)$ or (therapy $=1)$ or $($ mhealth $=1))$ then def_4 = 3; if $(($ func $=1))$ and $(($ serve $=1)$ or (therapy $=1)$ or $($ rxmeds $=1)$ or $($ mhealth $=1))$ then def_4 $=4$; if $(($ func $=1))$ and $(($ serve $=0)$ or (therapy $=0)$ or $($ rxmeds $=0)$ or $($ mhealth $=0))$ then def_ $4=4$; if def_4 $=0$ then def_4 = ; label def_4 = "CSHCN grouped according to type of qualifying criteria"; 


\begin{tabular}{|c|c|}
\hline Family Structure & $\begin{array}{l}\text { What are the family structure characteristics of the child's household at } \\
\text { the time of the survey? }\end{array}$ \\
\hline Survey Items Used & S1Q02, S9Q00, S9Q01, S9Q02 \\
\hline Numerators & $\begin{array}{l}\text { - Two-parent household (biological/adoptive) } \\
\text { - Two-parent stepfamily household } \\
\text { - Mother-only household, no father present } \\
\text { - Other family structure }\end{array}$ \\
\hline Denominator & Children/youth ages 0-17 years \\
\hline Interpretation & $\begin{array}{l}\text { Percent of children/youth ages 0-17 in two-parent households, two-parent } \\
\text { households with at least one step-parent, mother-only households with no } \\
\text { father of any type present, or in a household of any other family structure. }\end{array}$ \\
\hline \multicolumn{2}{|l|}{ Notes for Data-Users } \\
\hline \multicolumn{2}{|c|}{$\begin{array}{l}\text { To protect confidentiality, a single measure of family structure was created by the NCHS for inclusion in } \\
\text { the publicly released data file. "Family structure" refers to parents living in the household. Any of the } \\
\text { four family structure categories may include other people who act as parents, such as grandparents, } \\
\text { aunts, uncles, or unmarried partners of the parents. Legal guardians were not considered to be mothers } \\
\text { or fathers. Households identified as having two mothers of the same type (biological, step, foster, or } \\
\text { adoptive) were classified as "other family structure." However, because of this ambiguity about whether } \\
\text { the respondent was also counted as another parent in the household, these households may actually } \\
\text { be "single mother" households. Other households with ambiguous structure (e.g., where a father } \\
\text { refused to indicate whether he was the biological father) were also coded as "other family structure." }\end{array}$} \\
\hline \multicolumn{2}{|c|}{ SAS Code and Annotation } \\
\hline \multicolumn{2}{|l|}{ Format } \\
\hline \multicolumn{2}{|c|}{$\begin{array}{l}\text { value famtype } \\
\mathbf{1}=\text { = "Two-parent household (biological/adoptive)" } \\
\mathbf{2}=\text { "Two-parent household with at least one step-parent" } \\
\mathbf{3}=\text { "Mother-only household with no father of any type present" } \\
\mathbf{4}=\text { "All other family structures"; }\end{array}$} \\
\hline \multicolumn{2}{|l|}{ Code } \\
\hline
\end{tabular}




\begin{tabular}{|c|c|}
\hline Household Income & $\begin{array}{l}\text { How many children/youth live in households with incomes above/below } \\
\text { the Federal Poverty Level (FPL)? }\end{array}$ \\
\hline Survey Items Used & C11Q01; W9Q02 - W9Q12a \\
\hline Numerators & $\begin{array}{l}\text { - Household income less than } 100 \text { percent of FPL } \\
\text { - Household income 100-199 percent of FPL } \\
\text { - Household income 200-399 percent FPL } \\
\text { - Household income } 400 \text { percent FPL or more }\end{array}$ \\
\hline Denominator & Children/youth ages 0-17 years \\
\hline Interpretation & $\begin{array}{l}\text { Percent of children/youth ages 0-17 living in households whose income is } \\
\text { 0-99 percent, 100-199 percent, 200-399 percent, or } 400 \text { percent or more of } \\
\text { FPL. }\end{array}$ \\
\hline \multicolumn{2}{|l|}{ Notes for Data-Users } \\
\hline \multirow{3}{*}{\multicolumn{2}{|c|}{$\begin{array}{l}\text { Income level of household expressed as percent of FPL derived from total household income during } \\
\text { prior year and number of people living in the household. Federal Poverty Guidelines released in } 2002 \\
\text { and } 2003 \text { by the Department of Health and Human Services were used for the determination of } \\
\text { household poverty status. } \\
\text { For more information go to: http://www.cdc.gov/nchs/data/slaits/NSCH_Methodology_Report.pdf. } \\
\text { The number of unknown values for this variable is unusually high (10 percent). Unknown values are not } \\
\text { included in the denominator; weighted population counts may be underestimated. }\end{array}$}} \\
\hline & \\
\hline & \\
\hline \multicolumn{2}{|c|}{ SAS Code and Annotation } \\
\hline \multicolumn{2}{|l|}{ Format } \\
\hline \multicolumn{2}{|c|}{$\begin{array}{l}\text { value povlev } \\
\begin{aligned} \mathbf{1} & =\text { "HH income 0-99 percent FPL" } \\
\mathbf{2} & =\text { "HH income 100-199 percent FPL" } \\
\mathbf{3} & =\text { "HH income } 200-399 \text { percent FPL" } \\
\mathbf{4} & =\text { "HH income } 400 \text { percent FPL or more"; }\end{aligned}\end{array}$} \\
\hline \multicolumn{2}{|l|}{ Code } \\
\hline \multicolumn{2}{|c|}{ 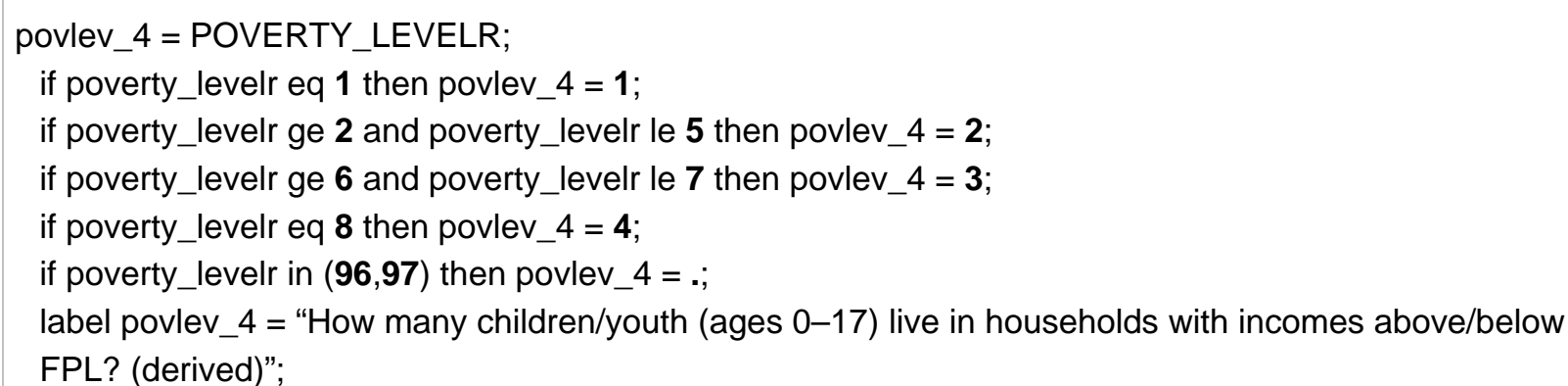 } \\
\hline
\end{tabular}




\begin{tabular}{|c|c|}
\hline $\begin{array}{l}\text { Insurance Type } \\
\text { (Indicator 3.3) }\end{array}$ & $\begin{array}{l}\text { What type of health insurance coverage, if any, did children/youth have } \\
\text { at the time of the survey? }\end{array}$ \\
\hline Survey Items Used & S3Q01, S3Q02 \\
\hline Numerators & $\begin{array}{l}\text { - } \quad \text { Children/youth who are currently uninsured } \\
\text { - } \quad \text { Children/youth who currently have private insurance coverage } \\
\text { - } \quad \text { Children/youth who currently have public insurance coverage }\end{array}$ \\
\hline Denominator & Children/youth ages 0-17 years \\
\hline Interpretation & $\begin{array}{l}\text { Children/youth ages } 0-17 \text { whose health insurance coverage at the time of } \\
\text { survey was either private }(\mathrm{S} 3 \mathrm{Q} 01=\text { Yes and S3Q02 = No) or public }(\mathrm{S} 3 \mathrm{Q} 01 \\
\text { and } \mathrm{S} 3 \mathrm{Q} 02=\mathrm{Yes}) \text { or none }(\mathrm{S} 3 \mathrm{Q} 01=\mathrm{No}) .\end{array}$ \\
\hline \multicolumn{2}{|l|}{ Notes for Data-Users } \\
\hline \multicolumn{2}{|c|}{$\begin{array}{l}\text { Private health insurance is defined as any type of health in } \\
\text { programs. Public health insurance is defined as Medicaid } \\
\text { Program (SCHIP). } \\
\text { This item serves as a stratifier as well as a key indicator. }\end{array}$} \\
\hline \multicolumn{2}{|c|}{ SAS Code and Annotation } \\
\hline \multicolumn{2}{|l|}{ Format } \\
\hline \multicolumn{2}{|c|}{$\begin{array}{l}\text { value insure /*ind3_1*/ } \\
\begin{aligned} \mathbf{1} & =\text { "Public insurance" } \\
\mathbf{2} & =\text { "Private insurance" } \\
\mathbf{3} & =\text { "Uninsured"; }\end{aligned}\end{array}$} \\
\hline \multicolumn{2}{|l|}{ Code } \\
\hline \multicolumn{2}{|c|}{ 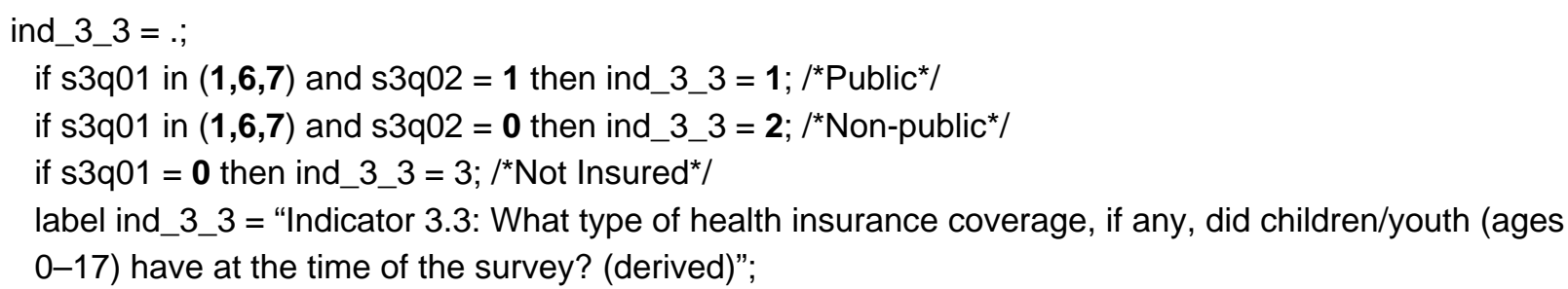 } \\
\hline
\end{tabular}




\begin{tabular}{|c|c|}
\hline $\begin{array}{l}\text { Insurance Status } \\
\text { (Indicator 3.1) }\end{array}$ & $\begin{array}{l}\text { Does (child's name) have any kind of health care coverage, including } \\
\text { health insurance, prepaid plans such as HMOs, or government plans } \\
\text { such as Medicaid? }\end{array}$ \\
\hline Survey Items Used & S3Q01 \\
\hline Numerator & Children/youth who currently have health care coverage. \\
\hline Denominator & Children/youth ages 0-17 years \\
\hline Interpretation & $\begin{array}{l}\text { Percent of children/youth ages } 0-17 \text { with any kind of health care coverage, } \\
\text { including health insurance, prepaid plans such as HMOs, or government } \\
\text { plans such as Medicaid (S3Q01 = Yes). }\end{array}$ \\
\hline \multicolumn{2}{|l|}{ Notes for Data-Users } \\
\hline \multicolumn{2}{|c|}{ This item serves as a stratifier as well as a key indicator. } \\
\hline \multicolumn{2}{|c|}{ SAS Code and Annotation } \\
\hline \multicolumn{2}{|l|}{ Format } \\
\hline \multicolumn{2}{|l|}{$\begin{array}{l}\text { value yn } \\
\qquad \begin{array}{l}\mathbf{1}=\text { "Yes" } \\
\mathbf{2}=\text { "No"; }\end{array}\end{array}$} \\
\hline \multicolumn{2}{|l|}{ Code } \\
\hline \multicolumn{2}{|c|}{$\begin{array}{l}\text { ind3_1 = ; } \\
\text { if s3q01 eq } 1 \text { then ind3_1=1; /*Insured*/ } \\
\text { if s3q01 = } 0 \text { then ind3_1 = 2; /*Not Insured*/ } \\
\text { label ind3_1 = "Indicator 3.1: Does (child's name) have any kind of health care coverage, including } \\
\text { health insurance, prepaid plans such as HMOs, or government plans such as Medicaid? (S3Q01)"; }\end{array}$} \\
\hline
\end{tabular}



Appendix A: Unweighted Frequency Table

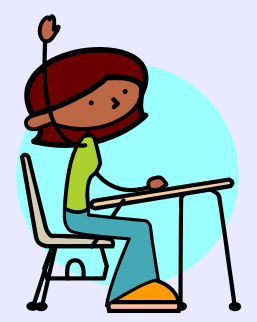


Indicator 1.1: In general, how would you describe (child's name)'s health? (S2Q01)

\begin{tabular}{|c|c|c|c|c|}
\hline ind1_1 & Frequency & Percent & $\begin{array}{r}\text { Cumulative } \\
\text { Frequency }\end{array}$ & $\begin{array}{c}\text { Cumulative } \\
\text { Percent }\end{array}$ \\
\hline Excellent/Very Good & 89155 & 87.13 & 89155 & 87.13 \\
\hline Good & 10680 & 10.44 & 99835 & 97.57 \\
\hline Fair/Poor & $\begin{array}{l}2486 \\
\text { Frequenc }\end{array}$ & $\begin{array}{c}2.43 \\
\text { issing }=\end{array}$ & 102321 & 100.00 \\
\hline
\end{tabular}

Indicator 1.2: How would you describe the condition of (child's name)'s teeth?(S2Q54 - ages 1-17 who have natural teeth)

\begin{tabular}{|c|c|c|c|c|}
\hline ind1_2 & Frequency & Percent & $\begin{array}{l}\text { Cumulative } \\
\text { Frequency }\end{array}$ & $\begin{array}{c}\text { Cumulative } \\
\text { Percent }\end{array}$ \\
\hline Excellent or very good condition & 69790 & 72.94 & 69790 & 72.94 \\
\hline Good condition & 18757 & 19.60 & 88547 & 92.55 \\
\hline Fair or poor condition & 7130 & 7.45 & 95677 & 100.00 \\
\hline
\end{tabular}

Indicator 1.3: Was (child's name) ever breastfed or fed breast milk? (S6Q59 -ages 0-5 only)

\begin{tabular}{ccccr} 
ind1_3 & Frequency & Percent & $\begin{array}{c}\text { Cumulative } \\
\text { Frequency }\end{array}$ & $\begin{array}{r}\text { Cumulative } \\
\text { Percent }\end{array}$ \\
\hline Yes & 24285 & 73.32 & 24285 & 73.32 \\
No & 8836 & 26.68 & 33121 & 100.00 \\
& Frequency Missing $=69232$ &
\end{tabular}

Indicator 1.4: What is the weight status of children/youth ages 10-17 based on BMI-for-age? (derived)

\begin{tabular}{|c|c|c|c|c|}
\hline ind1_4 & Frequency & Percent & $\begin{array}{r}\text { Cumulative } \\
\text { Frequency }\end{array}$ & $\begin{array}{c}\text { Cumulative } \\
\text { Percent }\end{array}$ \\
\hline Underweight & 2213 & 4.74 & 2213 & 4.74 \\
\hline Normal Weight & 30955 & 66.27 & 33168 & 71.01 \\
\hline At Risk for Overweight & 7119 & 15.24 & 40287 & 86.25 \\
\hline Overweight & 6420 & 13.75 & 46707 & 100.00 \\
\hline
\end{tabular}

Indicator 1.5: During the past week, on how many days did (child's name) exercise or participate in physical activity for at least 20 minutes that made him/her sweat and breathe hard? (S7Q21 - ages 6-17 only)

\begin{tabular}{lccrr} 
ind1_5 & Frequency & Percent & $\begin{array}{r}\text { Cumulative } \\
\text { Frequency }\end{array}$ & $\begin{array}{r}\text { Cumulative } \\
\text { Percent }\end{array}$ \\
\hline 0 days & 7705 & 11.27 & 7705 & 11.27 \\
$1-3$ days & 20695 & 30.28 & 28400 & 41.55 \\
$4-6$ days & 23332 & 34.14 & 51732 & 75.69 \\
Every day & 16617 & 24.31 & 68349 & 100.00 \\
& Frequency Missing $=34004$ &
\end{tabular}


Indicator 1.6: During the past 12 months, about how many days did (child's name) miss school because of illness or injury? (S7Q02 - ages 6-17 only)

\begin{tabular}{lcccr} 
ind1_6 & Frequency & Percent & $\begin{array}{r}\text { Cumulative } \\
\text { Frequency }\end{array}$ & $\begin{array}{r}\text { Cumulative } \\
\text { Percent }\end{array}$ \\
\hline 0 days & 14170 & 20.82 & 14170 & 20.82 \\
$1-5$ days & 41374 & 60.80 & 55544 & 81.62 \\
$6-10$ days & 8769 & 12.89 & 64313 & 94.51 \\
$11+$ days & 3737 & 5.49 & 68050 & 100.00 \\
& Frequency Missing $=34303$ &
\end{tabular}

\begin{tabular}{|c|c|c|c|c|}
\hline ind1_7 & Frequency & Percent & $\begin{array}{l}\text { Cumulative } \\
\text { Frequency }\end{array}$ & $\begin{array}{c}\text { Cumulative } \\
\text { Percent }\end{array}$ \\
\hline Yes & 3326 & 9.99 & 3326 & 9.99 \\
\hline No & 29964 & 90.01 & 33290 & 100.00 \\
\hline
\end{tabular}

Indicator 1.8: During the past 12 months, has (child's name) been poisoned by accident and required medical attention? (S6Q57 - ages 0-5; national-level data only)

\begin{tabular}{ccccc} 
ind1_8 & Frequency & Percent & $\begin{array}{c}\text { Cumulative } \\
\text { Frequency }\end{array}$ & $\begin{array}{c}\text { Cumulative } \\
\text { Percent }\end{array}$ \\
\hline Yes & 189 & 0.57 & 189 & 0.57 \\
No & 33109 & 99.43 & 33298 & 100.00 \\
& Frequency Missing & $=69055$ &
\end{tabular}

Indicator 1.11: Overall, would you say (child's name)'s asthma puts a burden on your family a great deal, a medium amount, a little, or not at all? (S2Q51 - only asked for children who currently have asthma)

\begin{tabular}{llccrr} 
& ind1_11 & Frequency & Percent & $\begin{array}{c}\text { Cumulative } \\
\text { Frequency }\end{array}$ & $\begin{array}{r}\text { Cumulative } \\
\text { Percent }\end{array}$ \\
\hline Affects family a great deal or medium amount & 1230 & 14.18 & 1230 & 14.18 \\
Affects family a little or not at all & 7445 & 85.82 & 8675 & 100.00
\end{tabular}

Indicator 1.12: During the past 12 months, has (child's name) stayed overnight in a hospital because of his/her asthma? (derived - national-level data only)

\begin{tabular}{|c|c|c|c|c|c|}
\hline & ind1_12 & Frequency & Percent & $\begin{array}{r}\text { Cumulative } \\
\text { Frequency }\end{array}$ & $\begin{array}{c}\text { Cumulative } \\
\text { Percent }\end{array}$ \\
\hline Have asthma & ever, no hospitalizations & 11728 & 11.48 & 11728 & 11.48 \\
\hline Have asthma & ever, yes hospitalized for asthma & 466 & 0.46 & 12194 & 11.94 \\
\hline Do not have & asthma & 89933 & 88.06 & 102127 & 100.00 \\
\hline
\end{tabular}

Frequency Missing $=226$ 
Indicator 2.1: How many children (ages 0-5) have parents with one or more concerns about child's learning, development, or behavior? (derived)

\begin{tabular}{|c|c|c|c|c|}
\hline ind2_1 & Frequency & Percent & $\begin{array}{l}\text { Cumulative } \\
\text { Frequency }\end{array}$ & $\begin{array}{c}\text { Cumulative } \\
\text { Percent }\end{array}$ \\
\hline 1 or more parent-reported concerns & 11695 & 35.10 & 11695 & 35.10 \\
\hline No parent-reported concerns & 21627 & 64.90 & 33322 & 100.00 \\
\hline 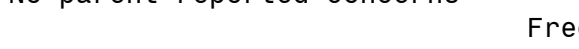 & ncy Missing & 69031 & & \\
\hline
\end{tabular}

Indicator 2.3: How many children/youth (ages 3-17) have moderate

or severe difficulties in the areas of emotions, concentration, behavior, or being able to get along with other people? (derived)

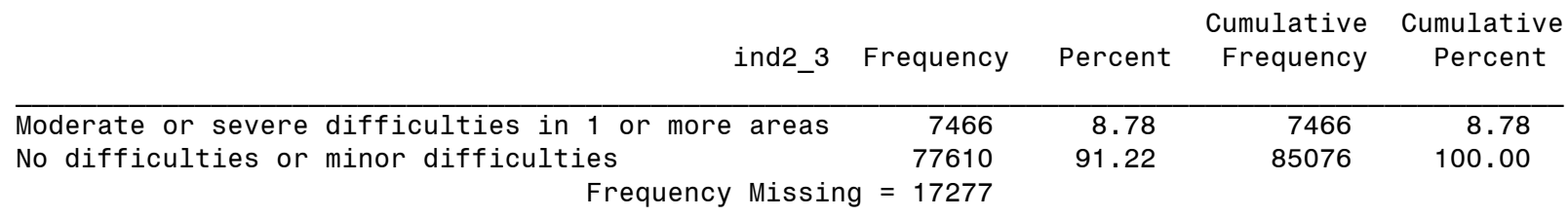

Indicator 2.4: Overall, would you say (child's name)'s mental and emotional health puts a burden on your family a great deal, a medium amount, a little, or not at all? (S2Q61 - ages 3-17 with socio-emotional difficulties)

\begin{tabular}{lcrrrr} 
& ind2_4 & Frequency & Percent & $\begin{array}{c}\text { Cumulative } \\
\text { Frequency }\end{array}$ & $\begin{array}{r}\text { Cumulative } \\
\text { Percent }\end{array}$ \\
\hline Affects family a great deal or medium amount & 4151 & 28.28 & 4151 & 28.28 \\
Affects family a little or not at all & 10527 & 71.72 & 14678 & 100.00
\end{tabular}

Indicator 2.6: How many children/youth (ages 6-17) often exhibit two or more problematic behaviors? (derived)

\begin{tabular}{|c|c|c|c|c|}
\hline ind2_6 & Frequency & Percent & $\begin{array}{l}\text { Cumulative } \\
\text { Frequency }\end{array}$ & $\begin{array}{c}\text { Cumulative } \\
\text { Percent }\end{array}$ \\
\hline Often exhibits 2 or more problematic behaviors & 4934 & 7.15 & 4934 & 7.15 \\
\hline Seldom exhibits problematic behaviors & 64097 & 92.85 & 69031 & 100.00 \\
\hline
\end{tabular}

Indicator 2.7: How many children/youth (ages 2-17) whose parents have ever been told child has ADD/ADHD currently take medication for this condition?

\begin{tabular}{|c|c|c|c|c|}
\hline ind2_7 & Frequency & Percent & $\begin{array}{l}\text { Cumulative } \\
\text { Frequency }\end{array}$ & $\begin{array}{c}\text { Cumulative } \\
\text { Percent }\end{array}$ \\
\hline ADD/ADHD ever; child currently takes medication & 3794 & 4.22 & 3794 & 4.22 \\
\hline ADD/ADHD ever; child not taking medication now & 2710 & 3.02 & 6504 & 7.24 \\
\hline Never told child has ADD/ADHD & $\begin{aligned} & 83326 \\
\mathrm{ng} & =12523\end{aligned}$ & 92.76 & 89830 & 100.00 \\
\hline
\end{tabular}




\begin{tabular}{|c|c|c|c|c|}
\hline ind2_8 & Frequency & Percent & $\begin{array}{r}\text { Cumulative } \\
\text { Frequency }\end{array}$ & $\begin{array}{c}\text { Cumulative } \\
\text { Percent }\end{array}$ \\
\hline Yes & 495 & 0.48 & 495 & 0.48 \\
\hline No & 101726 & 99.52 & 102221 & 100.00 \\
\hline
\end{tabular}

Indicator 3.1: Does (child's name) have any kind of health care coverage, including health insurance, prepaid plans such as HMOs, or government plans such as Medicaid? (S3Q01)

\begin{tabular}{ccccc} 
ind3_1 & Frequency & Percent & $\begin{array}{c}\text { Cumulative } \\
\text { Frequency }\end{array}$ & $\begin{array}{c}\text { Cumulative } \\
\text { Percent }\end{array}$ \\
\hline Yes & 94300 & 92.28 & 94300 & 92.28 \\
No & 7884 & 7.72 & 102184 & 100.00 \\
& \multicolumn{2}{c}{ Frequency Missing $=169$}
\end{tabular}

Indicator 3.2: How many children/youth (ages 0-17) had consistent health insurance coverage during the past 12 months? (derived)

\begin{tabular}{|c|c|c|c|c|}
\hline ind3_2 & Frequency & Percent & $\begin{array}{l}\text { Cumulative } \\
\text { Frequency }\end{array}$ & $\begin{array}{c}\text { Cumulative } \\
\text { Percent }\end{array}$ \\
\hline \multicolumn{5}{|l|}{ Currently uninsured or periods } \\
\hline of no coverage during year & 13280 & 13.02 & 13280 & 13.02 \\
\hline Consistently insured during past year & 88748 & 86.98 & 102028 & 100.00 \\
\hline
\end{tabular}

Indicator 3.3: What type of health insurance coverage, if any, did children/youth (ages $0-17$ ) have at the time of the survey? (derived)

\begin{tabular}{lccrr}
\multicolumn{1}{c}{ ind3_3 } & Frequency & Percent & $\begin{array}{r}\text { Cumulative } \\
\text { Frequency }\end{array}$ & $\begin{array}{c}\text { Cumulative } \\
\text { Percent }\end{array}$ \\
\hline Public insurance & 22624 & 22.33 & 22624 & 22.33 \\
Private insurance & 70793 & 69.88 & 93417 & 92.22 \\
Uninsured & 7884 & 7.78 & 101301 & 100.00 \\
& Frequency & Missing $=1052$ & &
\end{tabular}

Indicator 4.1: How many children/youth (ages 0-17) had one or more preventive medical care visits during the past 12 months? (derived)

\begin{tabular}{lcrrrr} 
& ind4_1 & Frequency & Percent & $\begin{array}{r}\text { Cumulative } \\
\text { Frequency }\end{array}$ & $\begin{array}{r}\text { Cumulative } \\
\text { Percent }\end{array}$ \\
\hline One or more preventive medical care visits & & 79190 & 78.06 & 79190 & 78.06 \\
NO preventive medical care visits & & 22255 & 21.94 & 101445 & 100.00
\end{tabular}


Indicator 4.2: How many children/youth (ages 1-17) with a need for routine preventive dental care, such as check-ups, screenings, and sealants, got all the care that was needed? (derived)

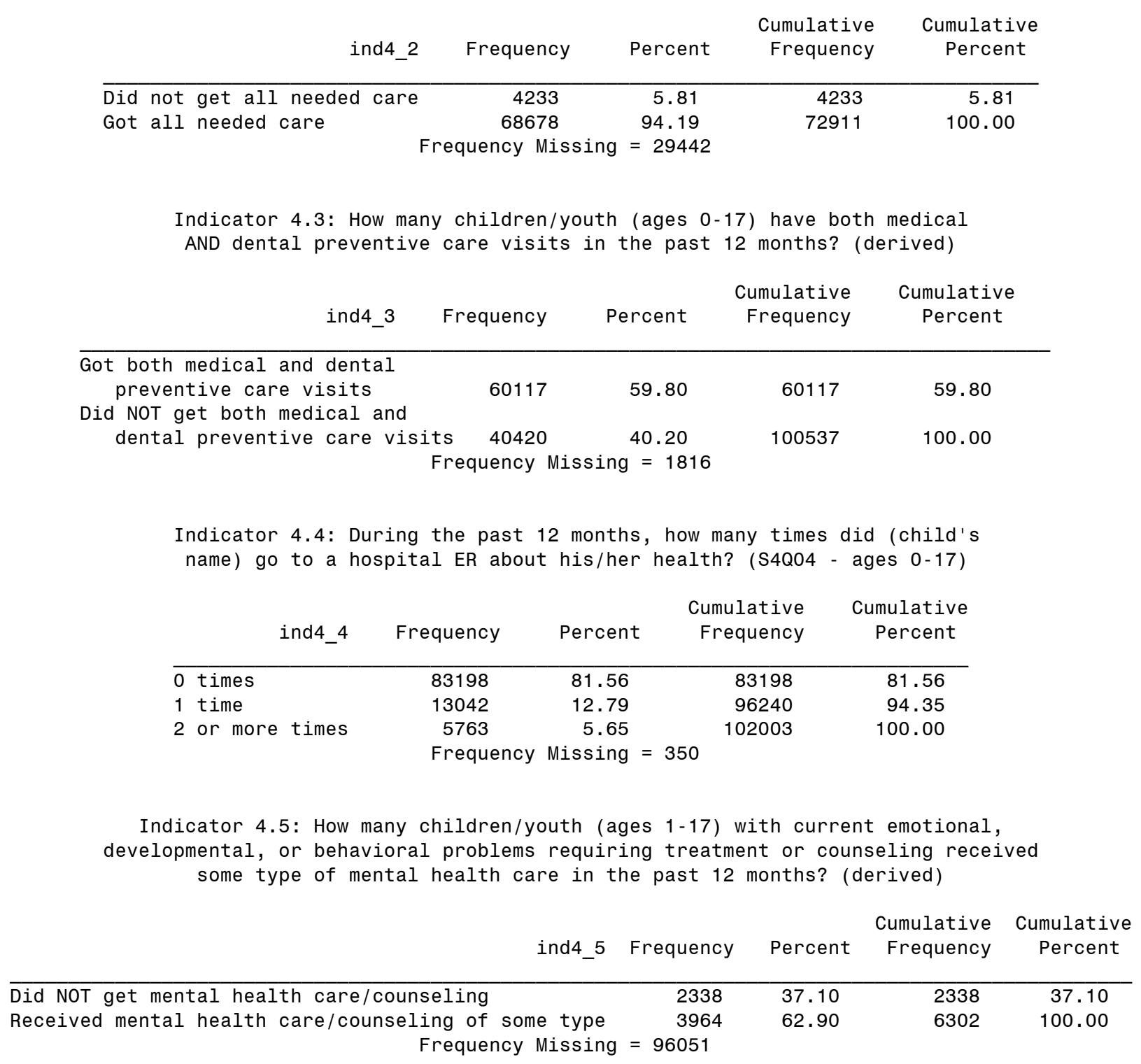

Indicator 4.6: During the past 12 months, did (child's name) receive all the medical care he/she needed? (S4Q07 - ages 0-17 who needed or used medical care)

\begin{tabular}{ccccr} 
ind4_6 & Frequency & Percent & $\begin{array}{r}\text { Cumulative } \\
\text { Frequency }\end{array}$ & $\begin{array}{r}\text { Cumulative } \\
\text { Percent }\end{array}$ \\
\hline Yes & 87853 & 98.66 & 87853 & 98.66 \\
No & 1192 & 1.34 & 89045 & 100.00 \\
& \multicolumn{2}{c}{ Frequency Missing $=13308$} &
\end{tabular}


Indicator 4.7: During the past 12 months, did (child's name) receive all the $\mathrm{Rx}$ he/she needed? (S4Q17 - ages 0-17 who needed $\mathrm{Rx}$ )

\begin{tabular}{|c|c|c|c|c|}
\hline ind4_7 & Frequency & Percent & $\begin{array}{l}\text { Cumulative } \\
\text { Frequency }\end{array}$ & $\begin{array}{l}\text { Cumulative } \\
\text { Percent }\end{array}$ \\
\hline Did not get all needed medication & 643 & 1.01 & 643 & 1.01 \\
\hline Got all needed medication & $\begin{array}{ll} & 63205 \\
\text { ncy } & \text { Missin }\end{array}$ & $\begin{array}{l}98.99 \\
38505\end{array}$ & 63848 & 100.00 \\
\hline
\end{tabular}

Indicator 4.8: How many children/youth (ages 0-17) receive health care that meets the AAP definition of medical home? (derived)

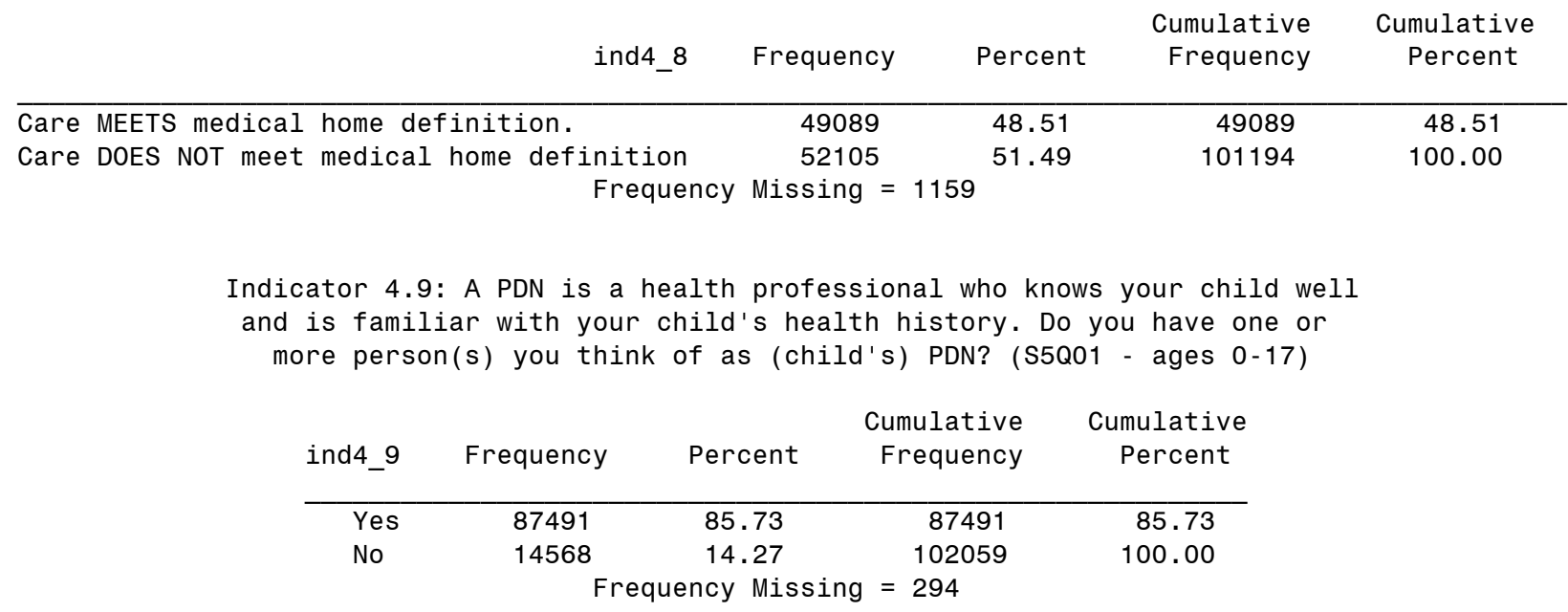

Indicator 4.10: How many children/youth (ages 0-17) have a PDN who consistently spends enough time with them and explains things in ways that children and parents can understand?

\begin{tabular}{|c|c|c|c|c|}
\hline ind4_10 & Frequency & Percent & $\begin{array}{l}\text { Cumulative } \\
\text { Frequency }\end{array}$ & $\begin{array}{l}\text { Cumulative } \\
\text { Percent }\end{array}$ \\
\hline $\begin{array}{l}\text { Child does not have a personal } \mathrm{dr} / \text { nurse } \\
\text { Personal } \mathrm{dr} / \text { nurse does not consistently }\end{array}$ & 14568 & 14.29 & 14568 & 14.29 \\
\hline $\begin{array}{l}\text { spend enough time or explain well } \\
\text { Personal dr/nurse consistently spends }\end{array}$ & 16760 & 16.44 & 31328 & 30.73 \\
\hline enough time and explains well & 70627 & 69.27 & 101955 & 100.00 \\
\hline
\end{tabular}

Frequency Missing $=398$ 
Indicator 4.11: How many children/youth (ages 0-17) have a PDN who is consistently available when phone advice or urgent care is needed for child? (derived - ages 0-17 who needed phone advice or urgent care)

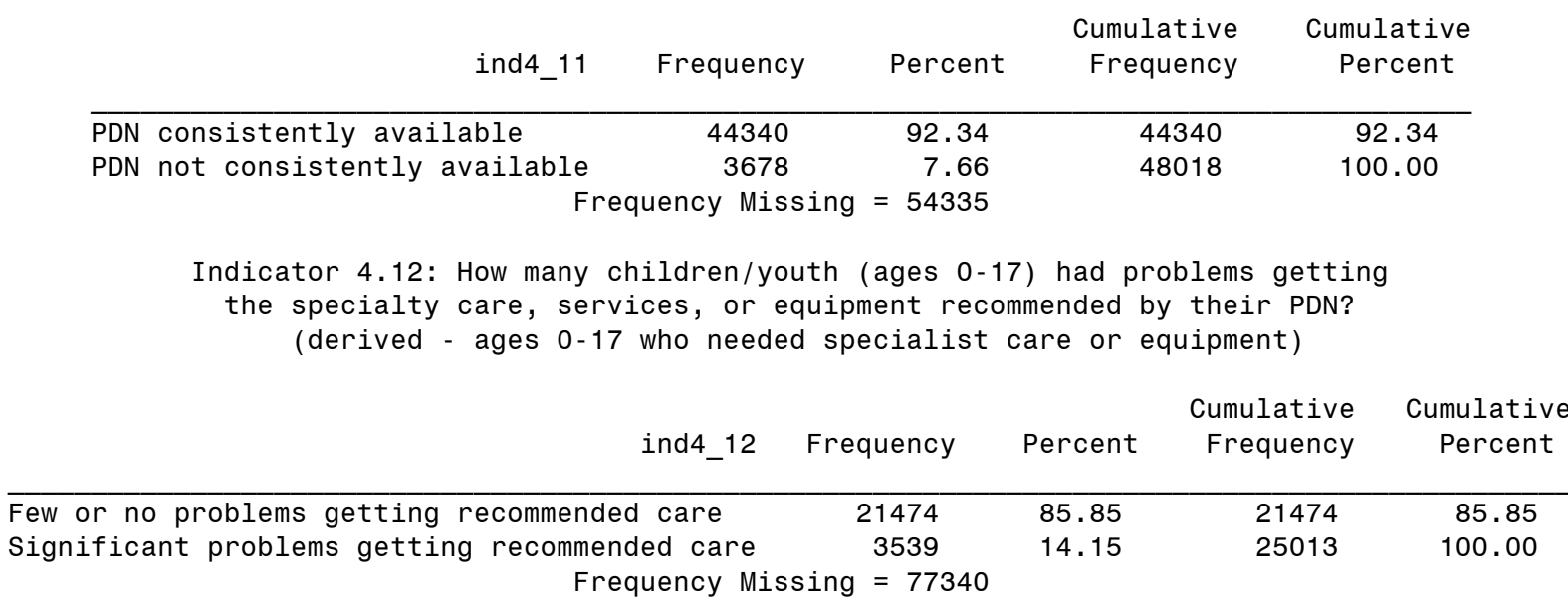

Indicator 4.13: How many children/youth (ages 0-17) have a PDN who follows up with family after child sees specialist or gets specialized services/equipment? (derived - ages 0-17 who got specialized services or equipment)

\begin{tabular}{|c|c|c|c|c|}
\hline ind4_13 & Frequency & Percent & $\begin{array}{r}\text { Cumulative } \\
\text { Frequency }\end{array}$ & $\begin{array}{c}\text { Cumulative } \\
\text { Percent }\end{array}$ \\
\hline PDN consistently follows up & 14214 & 57.71 & 14214 & 57.71 \\
\hline PDN does not consistently follow up & 10417 & 42.29 & 24631 & 100.00 \\
\hline
\end{tabular}

Indicator 4.14: During the past 12 months, did (child's name)'s doctors or other health care professionals ask if you have concerns about his/her learning, development, or behavior? (S6Q28 - ages 0-5)

\begin{tabular}{lccrr}
\multicolumn{1}{c}{ ind4_14 } & Frequency & Percent & $\begin{array}{c}\text { Cumulative } \\
\text { Frequency }\end{array}$ & $\begin{array}{c}\text { Cumulative } \\
\text { Percent }\end{array}$ \\
\hline Visited dr, asked about concerns & 13349 & 40.53 & 13349 & 40.53 \\
Visited dr, but not asked & 17397 & 52.82 & 30746 & 93.34 \\
Child did not visit doctor & $2193 \quad$ & 6.66 & 32939 & 100.00
\end{tabular}

Indicator 4.15: During the past 12 months, did (child's name)'s doctors give you specific information to address your concerns about his/her learning, development, or behavior? (S6Q29 - ages 0-5 with highly concerned parents)

\begin{tabular}{lcrrrr} 
& ind4_15 & Frequency & Percent & $\begin{array}{r}\text { Cumulative } \\
\text { Frequency }\end{array}$ & $\begin{array}{r}\text { Cumulative } \\
\text { Percent }\end{array}$ \\
\hline Highly concerned AND got needed info & 1719 & 45.85 & 1719 & 45.85 \\
Highly concerned but did not get info & $\begin{array}{r}2030 \\
\text { Frequency Missing }=98604\end{array}$ & 34.15 & 100.00
\end{tabular}


Indicator 5.1: How many children (ages 3-5) regularly attended nursery school, preschool, kindergarten, Head Start, or Early Start during the past month? (derived)

\begin{tabular}{|c|c|c|c|c|}
\hline ind5_1 & Frequency & Percent & $\begin{array}{r}\text { Cumulative } \\
\text { Frequency }\end{array}$ & $\begin{array}{c}\text { Cumulative } \\
\text { Percent }\end{array}$ \\
\hline Regularly attended during past month & 10197 & 62.45 & 10197 & 62.45 \\
\hline Did not attend during past month & 6130 & 37.55 & 16327 & 100.00 \\
\hline
\end{tabular}

Indicator 5.2: Since starting kindergarten, has (child's name) repeated any grades? (S7Q09 - ages 6-17)

\begin{tabular}{ccccr} 
ind5_2 & Frequency & Percent & $\begin{array}{r}\text { Cumulative } \\
\text { Frequency }\end{array}$ & $\begin{array}{r}\text { Cumulative } \\
\text { Percent }\end{array}$ \\
\hline Yes & 6580 & 9.55 & 6580 & 9.55 \\
No & 62301 & 90.45 & 68881 & 100.00 \\
& Frequency Missing $=33472$ &
\end{tabular}

Indicator 5.3: How many children/youth (ages 6-17) participate in one or more organized activities outside of school, such as sports teams or lessons, clubs, or religious groups? (derived)

\begin{tabular}{|c|c|c|c|c|}
\hline ind5_3 & Frequency & Percent & $\begin{array}{l}\text { Cumulative } \\
\text { Frequency }\end{array}$ & $\begin{array}{l}\text { Cumulative } \\
\text { Percent }\end{array}$ \\
\hline Participated in 1 or more organized activities & 58608 & 85.07 & 58608 & 85.07 \\
\hline $\begin{array}{r}\text { Did not participate in organized activities } \\
\text { Frequency } \mathrm{Mi}\end{array}$ & $\begin{array}{c}10284 \\
\text { sing }=3346\end{array}$ & 14.93 & 68892 & 100.00 \\
\hline
\end{tabular}

Indicator 5.4: During the past 12 months, has (child's name) been involved in any typeof community service or volunteer work at school, church, or in the community? (S7Q17 -ages 12-17)

\begin{tabular}{|c|c|c|c|c|}
\hline ind5_4 & Frequency & Percent & $\begin{array}{l}\text { Cumulative } \\
\text { Frequency }\end{array}$ & $\begin{array}{c}\text { Cumulative } \\
\text { Percent }\end{array}$ \\
\hline Participated in volunteer work & 24409 & 64.68 & 24409 & 64.68 \\
\hline Did NOT participate & $\begin{array}{c}13329 \\
\text { Iency Missi }\end{array}$ & $\begin{array}{r}35.32 \\
=64615\end{array}$ & 37738 & 100.00 \\
\hline
\end{tabular}

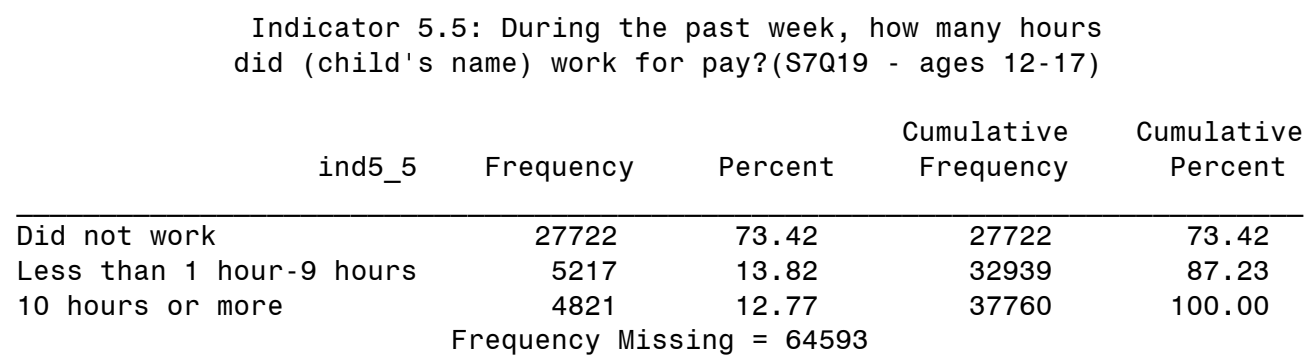


Indicator 5.6: On an average school day, about how much time does (child's name) usually spend reading for pleasure? (S7Q26 - ages 6-17)

\begin{tabular}{lcccr} 
ind5_6 & Frequency & Percent & $\begin{array}{c}\text { Cumulative } \\
\text { Frequency }\end{array}$ & $\begin{array}{c}\text { Cumulative } \\
\text { Percent }\end{array}$ \\
\hline None & 11635 & 17.17 & 11635 & 17.17 \\
30 minutes or less & 30872 & 45.55 & 42507 & 62.72 \\
$31-60$ minutes & 16876 & 24.90 & 59383 & 87.62 \\
Over 60 minutes & 8391 & 12.38 & 67774 & 100.00
\end{tabular}

Indicator 5.7: How often do children/youth (ages 0-17) attend religious services?(derived)

\begin{tabular}{|c|c|c|c|c|}
\hline ind5_7 & Frequency & Percent & $\begin{array}{l}\text { Cumulative } \\
\text { Frequency }\end{array}$ & $\begin{array}{c}\text { Cumulative } \\
\text { Percent }\end{array}$ \\
\hline Never & 23102 & 22.68 & 23102 & 22.68 \\
\hline At least once a year & 7273 & 7.14 & 30375 & 29.82 \\
\hline At least once a month & 17116 & 16.80 & 47491 & 46.62 \\
\hline Once a week, or more & $\begin{array}{c}54374 \\
\text { Frequency }\end{array}$ & $\begin{array}{l}53.38 \\
\text { ing }=488\end{array}$ & 101865 & 100.00 \\
\hline
\end{tabular}

\begin{tabular}{|c|c|c|c|c|}
\hline ind6_1 & Frequency & Percent & $\begin{array}{r}\text { Cumulative } \\
\text { Frequency }\end{array}$ & $\begin{array}{c}\text { Cumulative } \\
\text { Percent }\end{array}$ \\
\hline Excellent/Very Good & 66610 & 69.69 & 66610 & 69.69 \\
\hline Good & 20929 & 21.90 & 87539 & 91.59 \\
\hline Fair/Poor & 8042 & 8.41 & 95581 & 100.00 \\
\hline
\end{tabular}

Indicator 6.2: Would you say in general that (child's mother's name)'s mental and emotional health is excellent, very good, good, fair, or poor? (S9Q18

- biological, step, foster, or adoptive mother in the household)

\begin{tabular}{lcccr} 
ind6_2 & Frequency & Percent & $\begin{array}{c}\text { Cumulative } \\
\text { Frequency }\end{array}$ & $\begin{array}{c}\text { Cumulative } \\
\text { Percent }\end{array}$ \\
\hline Excellent/Very Good & 71717 & 75.08 & 71717 & 75.08 \\
Good & 18770 & 19.65 & 90487 & 94.73 \\
Fair/Poor & 5036 & 5.27 & 95523 & 100.00 \\
& Frequency & Missing $=6830$ & &
\end{tabular}


Indicator 6.3: How many children/youth (ages 0-17) have mothers whose physical and mental health were BOTH excellent or very good? (derived)

\begin{tabular}{|c|c|c|c|c|}
\hline ind6_3 & Frequency & Percent & $\begin{array}{l}\text { Cumulative } \\
\text { Frequency }\end{array}$ & $\begin{array}{l}\text { Cumulative } \\
\text { Percent }\end{array}$ \\
\hline $\begin{array}{l}\text { One or both are NOT excellent/very good } \\
\text { Maternal physical \& mental health BOTH }\end{array}$ & 35656 & 37.34 & 35656 & 37.34 \\
\hline excellent/very good & $\begin{array}{l}59822 \\
\text { Missing }=\end{array}$ & $75^{62.66}$ & 95478 & 100.00 \\
\hline
\end{tabular}

Indicator 6.4: Does anyone in the household use cigarettes, cigars, or pipe tobacco?

\begin{tabular}{|c|c|c|c|c|}
\hline ind6_4 & Frequency & Percent & $\begin{array}{r}\text { Cumulative } \\
\text { Frequency }\end{array}$ & $\begin{array}{c}\text { Cumulative } \\
\text { Percent }\end{array}$ \\
\hline Someone in household smokes tobacco & 26947 & 30.27 & 26947 & 30.27 \\
\hline No one in household smokes tobacco & 62085 & 69.73 & 89032 & 100.00 \\
\hline
\end{tabular}

Indicator 6.5: How many children/youth (ages 0-17) live in 'working poor' households - parents employed full-time with incomes less than 100 percent of the FPL?

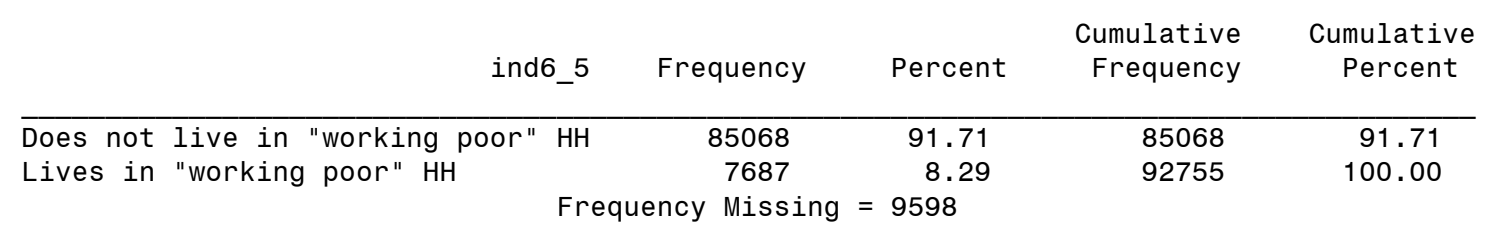

Indicator 6.6: During the past week, how many times did you or any family member
take (child's name) on any kind of outing, such as to the park, library, zoo,
shopping, church, restaurants, or family gatherings? (S8Q01 - ages 0-5 only)

\begin{tabular}{lcrrr} 
ind6_6 & Frequency & Percent & $\begin{array}{r}\text { Cumulative } \\
\text { Frequency }\end{array}$ & $\begin{array}{r}\text { Cumulative } \\
\text { Percent }\end{array}$ \\
\hline None & 819 & 2.47 & 819 & 2.47 \\
$1-3$ times & 11841 & 35.64 & 12660 & 38.11 \\
$4-6$ times & 12285 & 36.98 & 24945 & 75.09 \\
7 or more times & 8276 & 24.91 & 33221 & 100.00 \\
& Frequency Missing &
\end{tabular}


Indicator 6.7: During the past week, how many days did you or other family members read stories to (child's name)? (S6Q62 - ages 0-5 only)

\begin{tabular}{lcrrr} 
ind6_7 & Frequency & Percent & $\begin{array}{r}\text { Cumulative } \\
\text { Frequency }\end{array}$ & $\begin{array}{r}\text { Cumulative } \\
\text { Percent }\end{array}$ \\
\hline No days & 2171 & 6.55 & 2171 & 6.55 \\
1 or 2 days & 3753 & 11.32 & 5924 & 17.87 \\
3 or 4 days & 5942 & 17.93 & 11866 & 35.80 \\
5 or 6 days & 3962 & 11.95 & 15828 & 47.75 \\
Every day & 17318 & 52.25 & 33146 & 100.00 \\
& Frequency Missing $=69207$ &
\end{tabular}

Indicator 6.8: During the past week, on how many days did all the family members who live in the household eat a meal together? (S8Q03)

\begin{tabular}{lccrr} 
ind6_8 & Frequency & Percent & $\begin{array}{r}\text { Cumulative } \\
\text { Frequency }\end{array}$ & $\begin{array}{r}\text { Cumulative } \\
\text { Percent }\end{array}$ \\
\hline No days & 4698 & 4.60 & 4698 & 4.60 \\
$1-3$ days & 19909 & 19.48 & 24607 & 24.08 \\
$4-6$ days & 31379 & 30.71 & 55986 & 54.79 \\
Every day & 46203 & 45.21 & 102189 & 100.00 \\
& \multicolumn{2}{r}{ Frequency Missing $=164$}
\end{tabular}

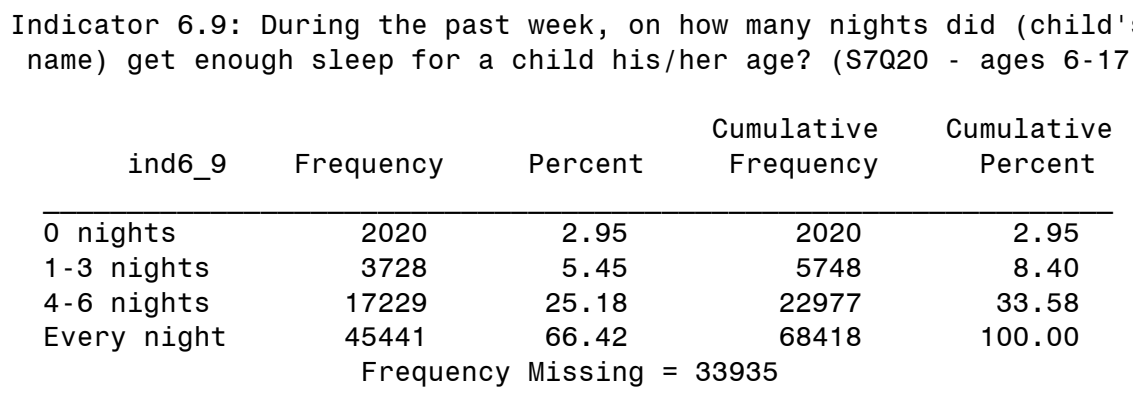

Indicator 6.10: On an average school day, about how many hours does (child's name) usually watch TV, watch videos, or play video games? (S7Q28 - ages 6-17 only)

\begin{tabular}{lccrr} 
ind6_10 & Frequency & Percent & $\begin{array}{r}\text { Cumulative } \\
\text { Frequency }\end{array}$ & $\begin{array}{r}\text { Cumulative } \\
\text { Percent }\end{array}$ \\
\hline None & 4827 & 7.04 & 4827 & 7.04 \\
1 hour or less & 33855 & 49.41 & 38682 & 56.46 \\
2 -3 hours & 25466 & 37.17 & 64148 & 93.62 \\
4 hours or more & 4369 & 6.38 & 68517 & 100.00 \\
& Frequency Missing $=33836$
\end{tabular}


Indicator 6.11: Sometimes children spend time caring for themselves at home without an adult responsible for them. During the past week, did (child's name) spend time caring for himself/herself for even a small amount of time? (S7Q15 - ages 6-11 only)

\begin{tabular}{ccccr} 
ind6_11 & Frequency & Percent & $\begin{array}{r}\text { Cumulative } \\
\text { Frequency }\end{array}$ & $\begin{array}{r}\text { Cumulative } \\
\text { Percent }\end{array}$ \\
\hline No & 25378 & 81.79 & 25378 & 81.79 \\
Yes & 5652 & 18.21 & 31030 & 100.00 \\
& Frequency & Missing $=71323$ &
\end{tabular}

Indicator 6.12: How many children/youth (ages 0-17) have parents who often felt aggravated with them during the past month?

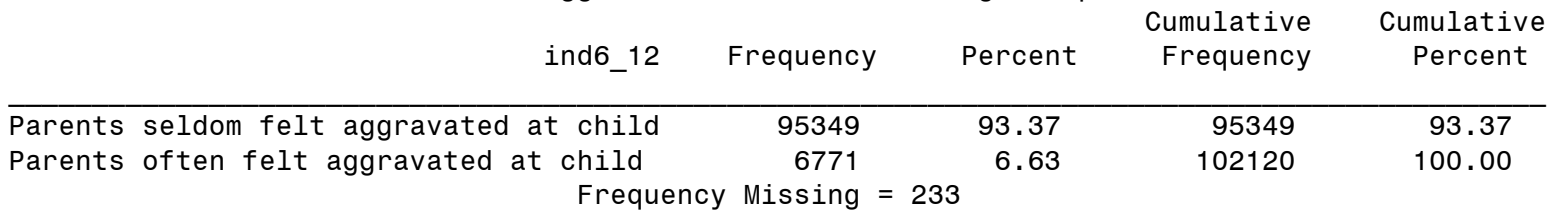

Indicator 6.13: During the past month, how many times have you had to make different arrangements for child care at the last minute because your usual plans changed due to circumstances beyond your control? (S6Q53 - ages 0-5)

\begin{tabular}{lccrr} 
ind6_13 & Frequency & Percent & $\begin{array}{r}\text { Cumulative } \\
\text { Frequency }\end{array}$ & $\begin{array}{c}\text { Cumulative } \\
\text { Percent }\end{array}$ \\
\hline None & 23827 & 71.75 & 23827 & 71.75 \\
1 time & 3537 & 10.65 & 27364 & 82.40 \\
2 -3 times & 4064 & 12.24 & 31428 & 94.64 \\
4 or more times & 1780 & 5.36 & 33208 & 100.00 \\
& Frequency Missing $=69145$ & &
\end{tabular}

Indicator 6.14: During the past 12 months, did you or anyone in the family have to quit, not take, or greatly change your job because of problems with child care for (child's name)? (S6Q54 - ages 0-5 only)

\begin{tabular}{ccccc} 
ind6_14 & Frequency & Percent & $\begin{array}{c}\text { Cumulative } \\
\text { Frequency }\end{array}$ & $\begin{array}{c}\text { Cumulative } \\
\text { Percent }\end{array}$ \\
\hline Yes & 3435 & 10.32 & 3435 & 10.32 \\
No & 29849 & 89.68 & 33284 & 100.00 \\
& Frequency Missing $=69069$ &
\end{tabular}

Indicator 6.15: How many children (ages 0-5) have parents who either had to make different child care arrangements in the past month or employment-related changes due to child care reasons in the past year or BOTH? (derived)

\begin{tabular}{lcrrrr} 
& ind6_15 & Frequency & Percent & $\begin{array}{c}\text { Cumulative } \\
\text { Frequency }\end{array}$ & $\begin{array}{c}\text { Cumulative } \\
\text { Percent }\end{array}$ \\
\hline One or more child care issues reported & 11170 & 33.64 & 11170 & 33.64 \\
No child care issues reported & & 22035 & 66.36 & 33205 & 100.00
\end{tabular}




\begin{tabular}{|c|c|c|c|c|}
\hline ind6_16 & Frequency & Percent & $\begin{array}{l}\text { Cumulative } \\
\text { Frequency }\end{array}$ & $\begin{array}{c}\text { Cumulative } \\
\text { Percent }\end{array}$ \\
\hline Yes, non-parental child care & 22384 & 67.19 & 22384 & 67.19 \\
\hline No non-parental child care & 10929 & 32.81 & 33313 & 100.00 \\
\hline
\end{tabular}

Indicator 7.1: How many children/youth (ages 0-17) live in supportive neighborhoods? (derived)

\begin{tabular}{|c|c|c|c|c|}
\hline ind7_1 & Frequency & Percent & $\begin{array}{r}\text { Cumulative } \\
\text { Frequency }\end{array}$ & $\begin{array}{c}\text { Cumulative } \\
\text { Percent }\end{array}$ \\
\hline Do not live in a supportive neighborhood & 16228 & 16.28 & 16228 & 16.28 \\
\hline Live in a supportive neighborhood & 83439 & 83.72 & 99667 & 100.00 \\
\hline
\end{tabular}

Indicator 7.2: How often do you feel (child's name) is safe in your community or neighborhood? (S10Q06)

\begin{tabular}{|c|c|c|c|c|}
\hline ind7_2 & Frequency & Percent & $\begin{array}{r}\text { Cumulative } \\
\text { Frequency }\end{array}$ & $\begin{array}{c}\text { Cumulative } \\
\text { Percent }\end{array}$ \\
\hline Usually or always safe & 88393 & 87.48 & 88393 & 87.48 \\
\hline Sometimes safe & 10556 & 10.45 & 98949 & 97.93 \\
\hline Never safe & $\begin{array}{c}2094 \\
\text { Frequency }\end{array}$ & $\begin{array}{l}2.07 \\
\text { ing }=1310\end{array}$ & 101043 & 100.00 \\
\hline
\end{tabular}

Indicator 7.3: How often do you feel (child's name) is safe at school? (S10Q07 -ages 6-17)

\begin{tabular}{|c|c|c|c|c|}
\hline ind7_3 & Frequency & Percent & $\begin{array}{l}\text { Cumulative } \\
\text { Frequency }\end{array}$ & $\begin{array}{c}\text { Cumulative } \\
\text { Percent }\end{array}$ \\
\hline Usually or always safe & 60723 & 91.07 & 60723 & 91.07 \\
\hline Sometimes safe & 5507 & 8.26 & 66230 & 99.33 \\
\hline Never safe & $\begin{array}{r}445 \\
\text { Frequency }\end{array}$ & $\begin{array}{c}0.67 \\
\text { ing }=35678\end{array}$ & 66675 & 100.00 \\
\hline
\end{tabular}

How many males and females are in the 0 - to 17 -year-old population? (S1Q01)

\begin{tabular}{lcccr} 
sex & Frequency & Percent & $\begin{array}{r}\text { Cumulative } \\
\text { Frequency }\end{array}$ & $\begin{array}{c}\text { Cumulative } \\
\text { Percent }\end{array}$ \\
\hline Male & 52554 & 51.39 & 52554 & 51.39 \\
Female & 49719 & 48.61 & 102273 & 100.00 \\
& & Frequency Missing $=80$ &
\end{tabular}


How many children/youth of different ages are in the 0 - to 17-year-old population? (derived - 3 groups)

\begin{tabular}{lrrrr} 
age_3 & Frequency & Percent & $\begin{array}{r}\text { Cumulative } \\
\text { Frequency }\end{array}$ & $\begin{array}{r}\text { Cumulative } \\
\text { Percent }\end{array}$ \\
\hline 0-5 yrs old & 33322 & 32.56 & 33322 & 32.56 \\
6-11 yrs old & 31117 & 30.40 & 64439 & 62.96 \\
$12-17$ yrs old & 37914 & 37.04 & 102353 & 100.00
\end{tabular}

How many children/youth of different ages are in the 0 - to 17-year-old population? (derived - 5 groups)

\begin{tabular}{lrrrr} 
age_5 & Frequency & Percent & $\begin{array}{r}\text { Cumulative } \\
\text { Frequency }\end{array}$ & $\begin{array}{c}\text { Cumulative } \\
\text { Percent }\end{array}$ \\
\hline $0-3$ yrs old & 22656 & 22.14 & 22656 & 22.14 \\
$4-7$ yrs old & 20755 & 20.28 & 43411 & 42.41 \\
$8-11$ yrs old & 21028 & 20.54 & 64439 & 62.96 \\
$12-14$ yrs old & 18174 & 17.76 & 82613 & 80.71 \\
$15-17$ yrs old & 19740 & 19.29 & 102353 & 100.00
\end{tabular}

How many children of different races or Hispanic ethnicity are in the 0 - to 17 -year-old population? (derived)

\begin{tabular}{lccrr} 
race_5 & Frequency & Percent & $\begin{array}{c}\text { Cumulative } \\
\text { Frequency }\end{array}$ & $\begin{array}{c}\text { Cumulative } \\
\text { Percent }\end{array}$ \\
\hline Hispanic & & & & \\
White, non-Hispanic & 73357 & 13.23 & 13357 & 13.23 \\
Black, non-Hispanic & 90104 & 69.45 & 83461 & 82.69 \\
Multiracial, non-Hispanic & 3946 & 9.50 & 93047 & 92.18 \\
Other, non-Hispanic & 3947 & 3.91 & 96990 & 96.09 \\
& Frequency Missing $=1416$ & 100937 & 100.00
\end{tabular}

Results for CSHCN screener

\begin{tabular}{lrrrr} 
metlwi & Frequency & Percent & $\begin{array}{r}\text { Cumulative } \\
\text { Frequency }\end{array}$ & $\begin{array}{r}\text { Cumulative } \\
\text { Percent }\end{array}$ \\
\hline CSHCN & 18578 & 18.15 & 18578 & 18.15 \\
Non-CSHCN & 83775 & 81.85 & 102353 & 100.00
\end{tabular}

How many children live in one-parent versus two-parent households? (derived)

\begin{tabular}{|c|c|c|c|c|}
\hline famtype & Frequency & Percent & $\begin{array}{l}\text { Cumulative } \\
\text { Frequency }\end{array}$ & $\begin{array}{r}\text { Cumulative } \\
\text { Percent }\end{array}$ \\
\hline Two-parent household (biological/adoptive) & 66185 & 66.27 & 66185 & 66.27 \\
\hline $\begin{array}{l}\text { Two-parent household with at least one step-parent } \\
\text { Mother-only household with no father of any }\end{array}$ & 8195 & 8.21 & 74380 & 74.47 \\
\hline type present & 20678 & 20.70 & 95058 & 95.17 \\
\hline All other family structures & 4820 & 4.83 & 99878 & 100.00 \\
\hline
\end{tabular}

Frequency Missing $=2475$ 
How many children/youth (ages 0-17) live in households with incomes above/below FPL? (derived)

\begin{tabular}{|c|c|c|c|c|c|c|}
\hline & & povlev_4 & Frequency & Percent & $\begin{array}{r}\text { Cumulative } \\
\text { Frequency }\end{array}$ & $\begin{array}{c}\text { Cumulative } \\
\text { Percent }\end{array}$ \\
\hline & income & 0-99 percent FPL & 11307 & 12.17 & 11307 & 12.17 \\
\hline $\mathrm{HH}$ & income & 100-199 percent FPL & 18850 & 20.28 & 30157 & 32.45 \\
\hline $\mathrm{HH}$ & income & 200-399 percent FPL & 33540 & 36.09 & 63697 & 68.54 \\
\hline $\mathrm{HH}$ & income & 400 percent FPL or more & 29242 & 31.46 & 92939 & 100.00 \\
\hline
\end{tabular}




\section{Appendix B: \\ Medical Home Component \& Interim Variables}

Unweighted frequency distributions for the Medical Home sub-components, related interim variables and composite measure generated by the SAS scoring program 


\section{Personal Doctor or Nurse (PDN) Component}

\section{**PDN sub-component score (S5Q01)}

Do you have one or more persons you think of as [S.C.]'s personal doctor or nurse?

\begin{tabular}{ccccc} 
S5Q01 & Frequency & Percent & $\begin{array}{c}\text { Cumulative } \\
\text { Frequency }\end{array}$ & $\begin{array}{c}\text { Cumulative } \\
\text { Percent }\end{array}$ \\
fffffffffffffffffffffffffffffffffffffffffffffffffffffff \\
0 & 14568 & 14.23 & 14568 & 14.23 \\
1 & 87491 & 85.48 & 102059 & 99.71 \\
6 & 267 & 0.26 & 102326 & 99.97 \\
7 & 26 & 0.03 & 102352 & 100.00 \\
& \multicolumn{3}{c}{ Frequency Missing $=1$}
\end{tabular}

\section{Family Centered Care Component}

How often does PDN spend enough time with them

$\begin{array}{ccccc}\text { adeqtime } & \text { Frequency } & \text { Percent } & \begin{array}{c}\text { Cumulative } \\ \text { Frequency }\end{array} & \begin{array}{c}\text { Cumulative } \\ \text { Percent }\end{array} \\ \text { fffffffffffffffffffffffffffffffffffffffffffffffffffffffffff} \\ \odot & 2006 & 2.31 & 2006 & 2.31 \\ 25 & 13379 & 15.39 & 15385 & 17.70 \\ 75 & 17414 & 20.04 & 32799 & 37.74 \\ 100 & 54117 & 62.26 & 86916 & 100.00 \\ & & & & \end{array}$

How often does PDN explain things so parent or ch understands

$\begin{array}{ccccc}\text { explain } & \text { Frequency } & \text { Percent } & \begin{array}{c}\text { Cumulative } \\ \text { Frequency }\end{array} & \begin{array}{c}\text { Cumulative } \\ \text { Percent }\end{array} \\ \text { fffffffffffffffffffffffffffffffffffffffffffffffffffffffff } \\ 0 & 756 & 0.87 & 756 & 0.87 \\ 25 & 3644 & 4.18 & 4400 & 5.05 \\ 75 & 12003 & 13.77 & 16403 & 18.81 \\ 100 & 70790 & 81.19 & 87193 & 100.00 \\ & & & \end{array}$




\section{**Interim variables for Communication with PDN sub-component}

\begin{tabular}{|c|c|c|c|c|}
\hline interprt & Frequency & Percent & $\begin{array}{r}\text { Cumulative } \\
\text { Frequency }\end{array}$ & $\begin{array}{c}\text { Cumulative } \\
\text { Percent }\end{array}$ \\
\hline \multicolumn{5}{|c|}{ ffffffffffffffffffffffffffffffffffffffffffffffffffffffffffff } \\
\hline$\odot$ & 138 & 12.33 & 138 & 12.33 \\
\hline 25 & 335 & 29.94 & 473 & 42.27 \\
\hline 75 & 99 & 8.85 & 572 & 51.12 \\
\hline $1 \odot \odot$ & 547 & 48.88 & 1119 & $1 \odot \odot . \odot \odot$ \\
\hline
\end{tabular}

Frequency Missing $=101234$

Number of PDN communication elements needed

$\begin{array}{ccccc}\text { pdn_cnt } & \text { Frequency } & \text { Percent } & \begin{array}{c}\text { Cumulative } \\ \text { Frequency }\end{array} & \begin{array}{c}\text { Cumulative } \\ \text { Percent }\end{array} \\ \text { ffffffffffffffffffffffffffffffffffffffffffffffffffffffffffff } \\ \odot & 14966 & 14.62 & 14966 & 14.62 \\ 1 & 643 & \odot .63 & 156 \odot 9 & 15.25 \\ 2 & 85647 & 83.68 & 101256 & 98.93 \\ 3 & 1097 & 1.07 & 102353 & 10 \odot . \odot \odot\end{array}$

Total points across all needed elements of comm w/ PDN

\begin{tabular}{|c|c|c|c|c|}
\hline pdn_sum & Frequency & Percent & $\begin{array}{r}\text { Cumulative } \\
\text { Frequency }\end{array}$ & $\begin{array}{c}\text { Cumulative } \\
\text { Percent }\end{array}$ \\
\hline \multicolumn{5}{|c|}{ fffffffffffffffffffffffffffffffffffffffffffffffffff} \\
\hline$\odot$ & 333 & 0.38 & 333 & 0.38 \\
\hline 25 & 711 & $\odot .81$ & 1044 & 1.19 \\
\hline $5 \odot$ & 1983 & 2.27 & $3 \odot 27$ & 3.46 \\
\hline 75 & 545 & $\odot .62$ & 3572 & 4.09 \\
\hline $10 \odot$ & $5 \odot 7 \odot$ & 5.80 & 8642 & 9.89 \\
\hline 125 & 8341 & 9.54 & 16983 & 19.43 \\
\hline 150 & 5286 & 6.05 & 22269 & 25.48 \\
\hline 175 & 14730 & 16.86 & 36999 & 42.34 \\
\hline $2 \odot \odot$ & 49820 & 57.01 & 86819 & 99.35 \\
\hline 225 & 203 & $\odot .23$ & $87 \odot 22$ & 99.58 \\
\hline $25 \odot$ & 34 & $\odot . \odot 4$ & 87056 & 99.62 \\
\hline 275 & $1 \odot \odot$ & 0.11 & 87156 & 99.74 \\
\hline $3 \odot \odot$ & 231 & $\odot .26$ & 87387 & $1 \odot \odot . \odot \odot$ \\
\hline
\end{tabular}




\begin{tabular}{|c|c|c|c|c|}
\hline pdn $1 \odot \odot$ & Frequency & Percent & $\begin{array}{r}\text { Cumulative } \\
\text { Frequency }\end{array}$ & $\begin{array}{c}\text { Cumulative } \\
\text { Percent }\end{array}$ \\
\hline \multicolumn{5}{|c|}{ ffffffffffffffffffffffffffffffffffffffffffffffffffffffffffff } \\
\hline$\odot$ & 333 & $\odot .38$ & 333 & $\odot .38$ \\
\hline 8.3 & $1 \odot$ & $\odot . \odot 1$ & 343 & 0.39 \\
\hline 12.5 & 622 & $\odot .71$ & 965 & 1.10 \\
\hline 16.7 & 34 & $\odot . \odot 4$ & 999 & 1.14 \\
\hline $25 . \odot$ & 2087 & 2.39 & 3086 & 3.53 \\
\hline 33.3 & 22 & $\odot .03$ & 3108 & 3.56 \\
\hline 37.5 & 367 & $\odot .42$ & 3475 & 3.98 \\
\hline 41.6 & 93 & 0.11 & 3568 & $4 . \odot 8$ \\
\hline $5 \odot . \odot$ & 4759 & 5.45 & 8327 & 9.53 \\
\hline 58.3 & 42 & $\odot . \odot 5$ & 8369 & 9.58 \\
\hline 62.5 & 8248 & 9.44 & 16617 & 19.02 \\
\hline 66.7 & 143 & $\odot .16$ & $1676 \odot$ & 19.18 \\
\hline $75 . \odot$ & 5486 & 6.28 & 22246 & 25.46 \\
\hline 83.3 & 34 & $\odot . \odot 4$ & 22280 & 25.50 \\
\hline 87.5 & 14688 & 16.81 & 36968 & 42.30 \\
\hline 91.7 & $10 \odot$ & 0.11 & 37068 & 42.42 \\
\hline $10 \odot . \odot$ & $5 \odot 319$ & 57.58 & 87387 & $1 \odot \odot . \odot \odot$ \\
\hline
\end{tabular}

\section{**Communication with PDN sub-component score (PDNCOM_2)}

PDN communication sub-component for MEDICAL HOME composite measure

\begin{tabular}{|c|c|c|c|c|}
\hline & & & $\begin{array}{l}\text { Cumulative } \\
\text { Frequency }\end{array}$ & $\begin{array}{c}\text { Cumulative } \\
\text { Percent }\end{array}$ \\
\hline \multicolumn{5}{|c|}{ 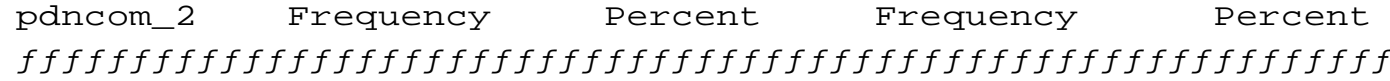 } \\
\hline$\odot$ & 16760 & 19.18 & $1676 \odot$ & 19.18 \\
\hline 1 & $7 \odot 627$ & $8 \odot .82$ & 87387 & $1 \odot \odot . \odot \odot$ \\
\hline
\end{tabular}




\section{Comprehensive Care Component}

** Preventive Medical Care Visit sub-component score (CARE_2)

\begin{tabular}{|c|c|c|c|c|}
\hline pc_2 & Frequency & Percent & $\begin{array}{l}\text { Cumulative } \\
\text { Frequency }\end{array}$ & $\begin{array}{c}\text { Cumulative } \\
\text { Percent }\end{array}$ \\
\hline \multicolumn{5}{|c|}{ ffffffffffffffffffffffffffffffffffffffffffffffffff } \\
\hline$\odot$ & $9 \odot 8$ & $\odot .89$ & $9 \odot 8$ & $\odot .89$ \\
\hline 1 & 22255 & 21.74 & 23163 & 22.63 \\
\hline 2 & 79190 & 77.37 & 102353 & $10 \odot . \odot \odot$ \\
\hline
\end{tabular}

**Interim variables Getting Needed Care sub-component

How often get advice over phone from ch's PDN when needed

$\begin{array}{ccccc}\text { phonehlp } & \text { Frequency } & \text { Percent } & \begin{array}{c}\text { Cumulative } \\ \text { Frequency }\end{array} & \begin{array}{c}\text { Cumulative } \\ \text { Percent }\end{array} \\ \text { ffffffffffffffffffffffffffffffffffffffffffffffffffffff } \\ \odot & 362 & 0.94 & 362 & 0.94 \\ 25 & 1682 & 4.35 & 2044 & 5.28 \\ 75 & 5416 & 14.00 & 7460 & 19.29 \\ 10 \odot & 31221 & 80.71 & 38681 & 100.00 \\ & & & & \end{array}$

How often get needed care right away from ch's PDN

$\begin{array}{ccccc}\text { getcare } & \text { Frequency } & \text { Percent } & \begin{array}{c}\text { Cumulative } \\ \text { Frequency }\end{array} & \begin{array}{c}\text { Cumulative } \\ \text { Percent }\end{array} \\ \text { ffffffffffffffffffffffffffffffffffffffffffffffffffffffffffff} \\ \Theta & 553 & 2.02 & 553 & 2.02 \\ 25 & 1572 & 5.75 & 2125 & 7.78 \\ 75 & 5120 & 18.74 & 7245 & 26.52 \\ 100 & 20074 & 73.48 & 27319 & 100.00\end{array}$

Frequency Missing $=75034$ 
Number of getting care elements needed

$\begin{array}{ccccc}\text { care_cnt } & \text { Frequency } & \text { Percent } & \begin{array}{c}\text { Cumulative } \\ \text { Frequency }\end{array} & \begin{array}{c}\text { Cumulative } \\ \text { Percent }\end{array} \\ \text { fffffffffffffffffffffffffffffffffffffffffffffffffffffffffffff }\end{array}$

Total points across needed elements of getting phone or urgent care from PDN

$\begin{array}{ccccc}\text { care_sum } & \text { Frequency } & \text { Percent } & \begin{array}{c}\text { Cumulative } \\ \text { Frequency }\end{array} & \begin{array}{c}\text { Cumulative } \\ \text { Percent }\end{array} \\ \text { ffffffffffffffffffffffffffffffffffffffffffffffffffffffff }\end{array}$

Average score for Getting Needed Care sub-component of MEDICAL HOME composite measure

\begin{tabular}{|c|c|c|c|c|}
\hline care100 & Frequency & Percent & $\begin{array}{r}\text { Cumulative } \\
\text { Frequency }\end{array}$ & $\begin{array}{c}\text { Cumulative } \\
\text { Percent }\end{array}$ \\
\hline \multicolumn{5}{|c|}{ ffffffffffffffffffffffffffffffffffffffffffffffffffff} \\
\hline$\odot$ & 472 & $\odot .98$ & 472 & 0.98 \\
\hline 12.5 & 132 & $\odot .27$ & $6 \odot 4$ & 1.26 \\
\hline $25 . \odot$ & 1726 & 3.59 & 2330 & 4.85 \\
\hline 37.5 & 92 & $\odot .19$ & 2422 & 5.04 \\
\hline $5 \odot . \odot$ & $6 \odot 9$ & 1.27 & 3031 & 6.31 \\
\hline 62.5 & 647 & 1.35 & 3678 & 7.66 \\
\hline $75 . \odot$ & 5692 & 11.85 & 9370 & 19.51 \\
\hline 87.5 & 3204 & 6.67 & 12574 & 26.19 \\
\hline $10 \odot . \odot$ & 35444 & 73.81 & 48018 & $10 \odot . \odot \odot$ \\
\hline
\end{tabular}




\section{${ }^{\star *}$ Getting Needed Care sub-component score (CARE_2)}

Getting Needed Care sub-component for MEDICAL HOME composite measure

$\begin{array}{ccccc} & & \text { Cumulative } & \text { Cumulative } \\ \text { care_2 } & \text { Frequency } & \text { Percent } & \text { Frequency } & \text { Percent } \\ \text { fffffffffffffffffffffffffffffffffffffffffffffffffffffffffff } \\ \odot & 3678 & 7.66 & 3678 & 7.66 \\ 1 & 44340 & 92.34 & 48018 & 100.0 \odot\end{array}$

Frequency Missing $=54335$

**Interim variables Access to Specialist Care \& Services sub-component

\begin{tabular}{|c|c|c|c|c|}
\hline special & Frequency & Percent & $\begin{array}{l}\text { Cumulative } \\
\text { Frequency }\end{array}$ & $\begin{array}{c}\text { Cumulative } \\
\text { Percent }\end{array}$ \\
\hline \multicolumn{5}{|c|}{ } \\
\hline 0 & 966 & 4.48 & 966 & 4.48 \\
\hline 25 & 1593 & 7.39 & 2559 & 11.88 \\
\hline 75 & 2118 & 9.83 & 4677 & 21.71 \\
\hline $10 \odot$ & 16870 & 78.29 & 21547 & $1 \odot \odot . \odot \odot$ \\
\hline \multicolumn{5}{|c|}{ Frequency Missing $=80806$} \\
\hline \multicolumn{5}{|c|}{ Problems getting needed services or equip } \\
\hline services & Frequency & Percent & $\begin{array}{l}\text { Cumulative } \\
\text { Frequency }\end{array}$ & $\begin{array}{c}\text { Cumulative } \\
\text { Percent }\end{array}$ \\
\hline \multicolumn{5}{|c|}{ fffffffffffffffffffffffffffffffffffffffffffffffffffffffffffff } \\
\hline$\odot$ & 638 & 6.96 & 638 & 6.96 \\
\hline 25 & 782 & 8.54 & 1420 & $15.5 \odot$ \\
\hline 75 & $1 \odot 36$ & 11.31 & 2456 & 26.81 \\
\hline $10 \odot$ & $67 \odot 5$ & 73.19 & 9161 & $10 \odot . \odot \odot$ \\
\hline
\end{tabular}




\begin{tabular}{ccccc}
\multicolumn{5}{c}{ Number of access to care elements needed } \\
acc_cnt & Frequency & Percent & $\begin{array}{c}\text { Cumulative } \\
\text { Frequency }\end{array}$ & $\begin{array}{c}\text { Cumulative } \\
\text { Percent }\end{array}$ \\
ffffffffffffffffffffffffffffffffffffffffffffffffffff \\
$\Theta$ & 77340 & 75.56 & 77340 & 75.56 \\
1 & 19318 & 18.87 & 96658 & 94.44 \\
2 & 5695 & 5.56 & 102353 & $100.0 \odot$
\end{tabular}

Total points across accessing needed specialist care/services elements of MEDICAL HOME

$\begin{array}{ccccc}\text { acc_sum } & \text { Frequency } & \text { Percent } & \begin{array}{c}\text { Cumulative } \\ \text { Frequency }\end{array} & \begin{array}{c}\text { Cumulative } \\ \text { Percent }\end{array} \\ \text { fffffffffffffffffffffffffffffffffffffffffffffffffffffff }\end{array}$

Average score for access to needed specialist care/services sub-component of MEDICAL HOME

\begin{tabular}{|c|c|c|c|c|}
\hline $\operatorname{acc} 100$ & Frequency & Percent & $\begin{array}{l}\text { Cumulative } \\
\text { Frequency }\end{array}$ & $\begin{array}{c}\text { Cumulative } \\
\text { Percent }\end{array}$ \\
\hline \multicolumn{5}{|c|}{ ffffffffffffffffffffffffffffffffffffffffffffffffffffff} \\
\hline$\odot$ & 918 & 3.67 & 918 & 3.67 \\
\hline 12.5 & 127 & $\odot .51$ & 1045 & 4.18 \\
\hline $25 . \odot$ & 1475 & $5.9 \odot$ & 2520 & 10.07 \\
\hline 37.5 & 109 & $\odot .44$ & 2629 & 10.51 \\
\hline $5 \odot . \odot$ & 457 & 1.83 & 3086 & 12.34 \\
\hline 62.5 & 453 & 1.81 & 3539 & 14.15 \\
\hline $75 . \odot$ & 1983 & 7.93 & 5522 & 22.08 \\
\hline 87.5 & 680 & 2.72 & 6202 & 24.80 \\
\hline 100.0 & 18811 & 75.20 & 25013 & 100.00 \\
\hline
\end{tabular}


${ }^{\star \star}$ Access to Specialist Care \& Services sub-component score (ACC_2)

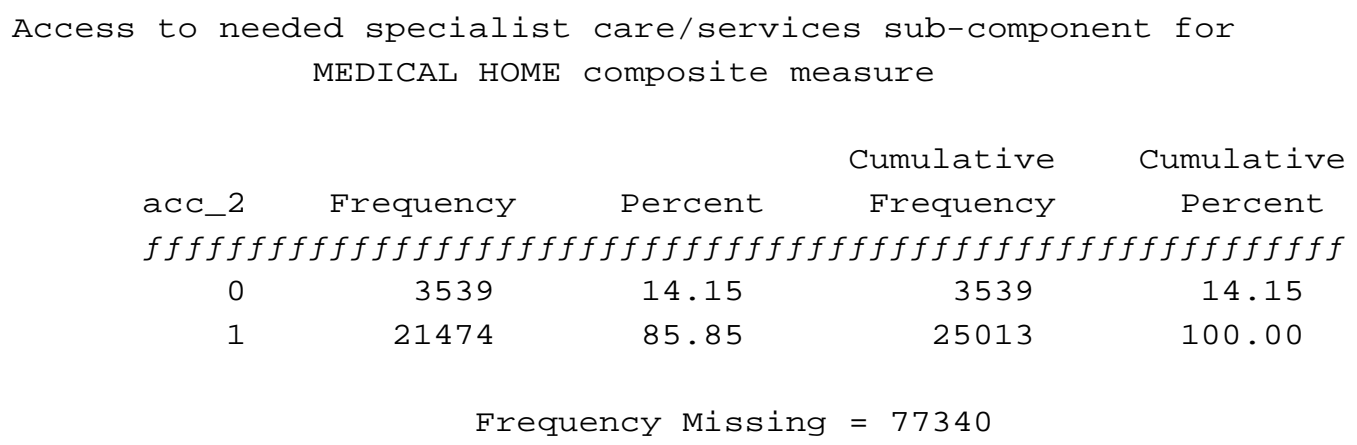

\section{Coordinated Care Component}

**Interim variables Follow Up with Family sub-component

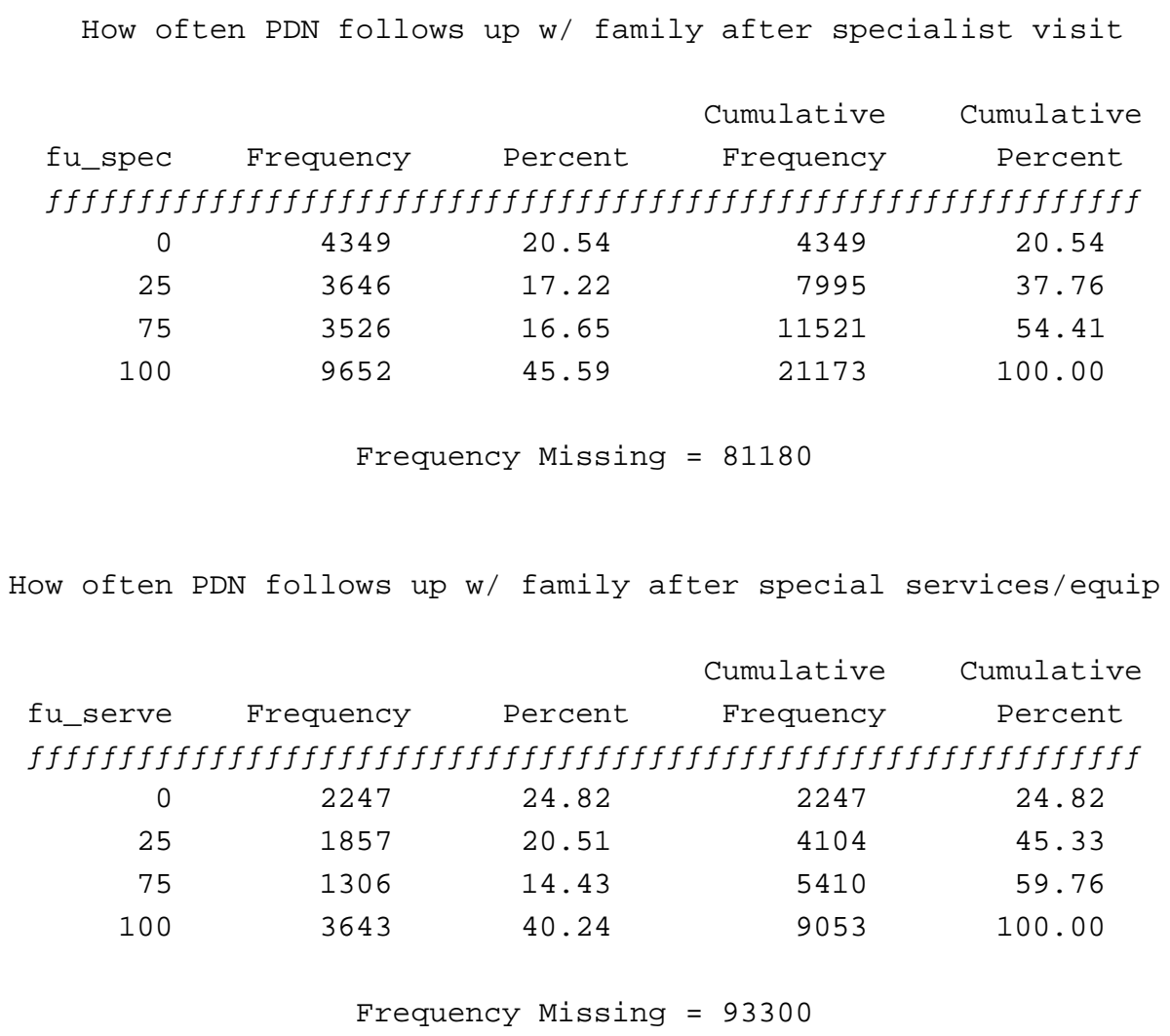


Number of coor care follow up components

$\begin{array}{ccccc}\text { coor_cnt } & \text { Frequency } & \text { Percent } & \begin{array}{c}\text { Cumulative } \\ \text { Frequency }\end{array} & \begin{array}{c}\text { Cumulative } \\ \text { Percent }\end{array} \\ \text { fffffffffffffffffffffffffffffffffffffffffffffffffffffffffffff } \\ \odot & 77722 & 75.94 & 77722 & 75.94 \\ 1 & 19036 & 18.60 & 96758 & 94.53 \\ 2 & 5595 & 5.47 & 102353 & 100.0 \odot\end{array}$

Total points on needed follow up care elements of MEDICAL HOME

$\begin{array}{ccccc}\text { coor_sum } & \text { Frequency } & \text { Percent } & \begin{array}{c}\text { Cumulative } \\ \text { Frequency }\end{array} & \begin{array}{c}\text { Cumulative } \\ \text { Percent }\end{array} \\ \text { fffffffffffffffffffffffffffffffffffffffffffffffffffffffffff } \\ \odot & 4969 & 2 \odot .17 & 4969 & 20.17 \\ 25 & 3698 & 15.01 & 8667 & 35.19 \\ 5 \odot & 547 & 2.22 & 9214 & 37.41 \\ 75 & 313 \odot & 12.71 & 12344 & 5 \odot .12 \\ 10 \odot & 9208 & 37.38 & 21552 & 87.5 \odot \\ 125 & 369 & 1.5 \odot & 21921 & 89.0 \odot \\ 15 \odot & 437 & 1.77 & 22358 & 9 \odot .77 \\ 175 & 486 & 1.97 & 22844 & 92.74 \\ 20 \odot & 1787 & 7.26 & 24631 & 10 \odot .0 \odot \\ & & & & \end{array}$

Average score on FOLLOW UP W/ FAMILY coor care sub-component Of MEDICAL HOME

$\begin{array}{ccccc}\text { coor } 10 \odot & \text { Frequency } & \text { Percent } & \begin{array}{c}\text { Cumulative } \\ \text { Frequency }\end{array} & \begin{array}{c}\text { Cumulative } \\ \text { Percent }\end{array} \\ \text { ffffffffffffffffffffffffffffffffffffffffffffffffffffff } \\ \odot & 4969 & 2 \odot .17 & 4969 & 2 \odot .17 \\ 12.5 & 456 & 1.85 & 5425 & 22.03 \\ 25 . \odot & 3789 & 15.38 & 9214 & 37.41 \\ 37.5 & 155 & \odot .63 & 9369 & 38.04 \\ 5 \odot . \odot & 679 & 2.76 & 10 \odot 48 & 4 \odot .79 \\ 62.5 & 369 & 1.5 \odot & 10417 & 42.29 \\ 75 . \odot & 3412 & 13.85 & 13829 & 56.14 \\ 87.5 & 486 & 1.97 & 14315 & 58.12 \\ 10 \odot . \odot & 10316 & 41.88 & 24631 & 10 \odot . \odot \odot \\ & & & & \end{array}$


${ }^{\star *}$ Follow Up with Family sub-component score (COOR_2)

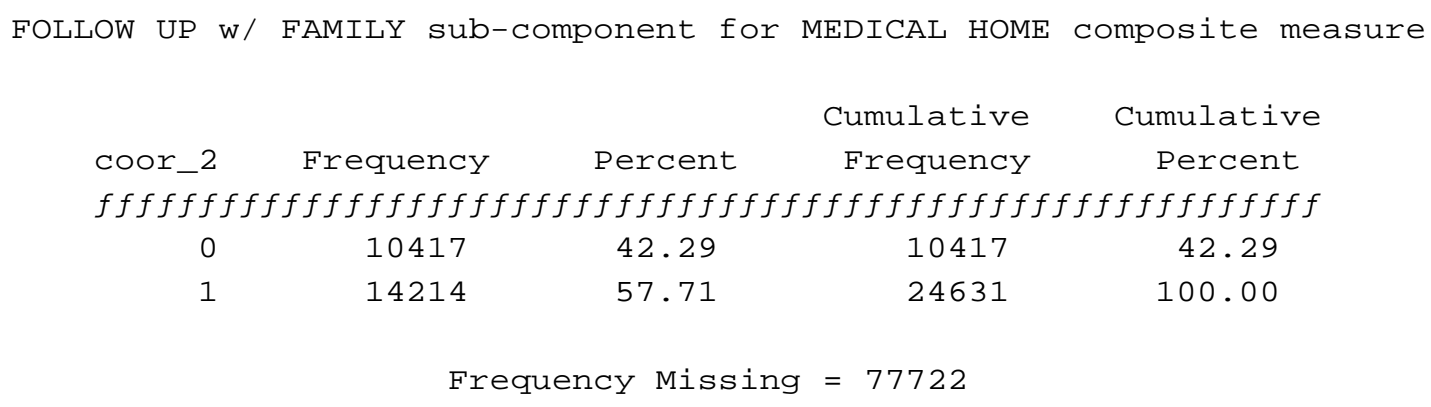

\section{Medical Home Composite Measure}

${ }^{*}$ Interim variables for Medical Home Composite Measure

\begin{tabular}{ccccc}
\multicolumn{4}{c}{ Number of needed sub-components of Medical Home } \\
mh_cnt & Frequency & Percent & $\begin{array}{c}\text { Cumulative } \\
\text { Frequency }\end{array}$ & $\begin{array}{c}\text { Cumulative } \\
\text { Percent }\end{array}$ \\
ffffffffffffffffffffffffffffffffffffffffffffffffffff \\
0 & 14941 & 14.60 & 14941 & 14.60 \\
1 & 32130 & 31.39 & 47071 & 45.99 \\
2 & 30402 & 29.70 & 77473 & 75.69 \\
3 & 7405 & 7.23 & 84878 & 82.93 \\
4 & 17475 & 17.07 & 102353 & 100.00
\end{tabular}

Number needed Medical Home sub-components with score of $75 p t s$ or above

$\begin{array}{ccccc}\text { mh_cnt2 } & \text { Frequency } & \text { Percent } & \begin{array}{c}\text { Cumulative } \\ \text { Frequency }\end{array} & \begin{array}{c}\text { Cumulative } \\ \text { Percent }\end{array} \\ \text { ffffffffffffffffffffffffffffffffffffffffffffffffffffffffffff } \\ \odot & 24383 & 23.82 & 24383 & 23.82 \\ 1 & 30895 & 30.18 & 55278 & 54.01 \\ 2 & 29831 & 29.15 & 85109 & 83.15 \\ 3 & 8878 & 8.67 & 93987 & 91.83 \\ 4 & 8366 & 8.17 & 102353 & 100.00\end{array}$




\title{
**Medical Home Composite Measure score (MH_COMP)
}

\author{
How many children/youth (ages $0-17$ ) receive health \\ care meeting the AAP definition of medical home?

$\begin{array}{ccccc} & & \text { Cumulative } & \text { Cumulative } \\ \text { mh_comp } & \text { Frequency } & \text { Percent } & \text { Frequency } & \text { Percent } \\ \text { ffffffffffffffffffffffffffffffffffffffffffffffffffffffffffff } \\ 1 & 49089 & 48.51 & 49089 & 48.51 \\ 2 & 52105 & 51.49 & 101194 & 100.00\end{array}$ \\ Frequency Missing $=1159$
}

Percent of needed MEDICAL HOME sub-components with score $75 p t s$ or above

\begin{tabular}{|c|c|c|c|c|}
\hline mh2_scor & Frequency & Percent & $\begin{array}{l}\text { Cumulative } \\
\text { Frequency }\end{array}$ & $\begin{array}{r}\text { Cumulati } \\
\text { Percen }\end{array}$ \\
\hline \multicolumn{5}{|c|}{ ffffffffffffffffffffffffffffffffffffffffffffffffffffffffff } \\
\hline 0 & 9442 & 10.80 & 9442 & 10.80 \\
\hline $25 . \odot$ & 720 & $\odot .82$ & 10162 & 11.6 \\
\hline 33.3 & 1262 & 1.44 & 11424 & 13.0 \\
\hline 50.0 & 7115 & 8.14 & 18539 & 21.2 \\
\hline 66.7 & 3038 & 3.48 & 21577 & 24. \\
\hline 75.0 & 5970 & 6.83 & 27547 & 31.5 \\
\hline 100.0 & 59865 & 68.49 & 87412 & $100 . c$ \\
\hline \multicolumn{5}{|c|}{ Frequency Missing = 14941} \\
\hline \multicolumn{5}{|c|}{$\begin{array}{l}\% \text { meet MED HOME threshold of } 75 p t \text { or above on EVERY } \\
\text { needed sub-component - prev care visit NOT included }\end{array}$} \\
\hline mh_yn & Frequency & Percent & $\begin{array}{l}\text { Cumulative } \\
\text { Frequency }\end{array}$ & $\begin{array}{l}\text { Cumulative } \\
\text { Percent }\end{array}$ \\
\hline \multicolumn{5}{|c|}{ fffffffffffffffffffffffffffffffffffffffffffffffffffff } \\
\hline 0 & 42195 & 41.34 & 42195 & 41.34 \\
\hline 1 & 59865 & 58.66 & 102060 & $10 \odot . \odot \odot$ \\
\hline
\end{tabular}

